\title{
Supporting information : \\ N-Alkenyl-2-aziridinylmethyl Radicals and N-Alkenylaminyl Radicals in Cascade Cyclizations to Pyrrolizidines and Indolizidines
}

\author{
V. Van Speybroeck, N. De Kimpe, M. Waroquier
}

Contribution from the Laboratory of Theoretical Physics, Ghent University,

Proeftuinstraat 86, B-9000 Ghent, Belgium

and

the Department of Organic Chemistry, Faculty of Bioscience

Engineering, Ghent University, Coupure Links 653, B-9000 Ghent,

Belgium

1. Energetic results at the B3LYP/6-311g** level of theory for the $\mathrm{S} 1$ formation of pyrrolizidines

2. Z-matrices of all optimized structures at the B3LYP/6-311G** level of theory S2

3. Energetic Results

\section{Energetic results at the B3LYP/6-311g** level of theory for the formation of pyrrolizidines}

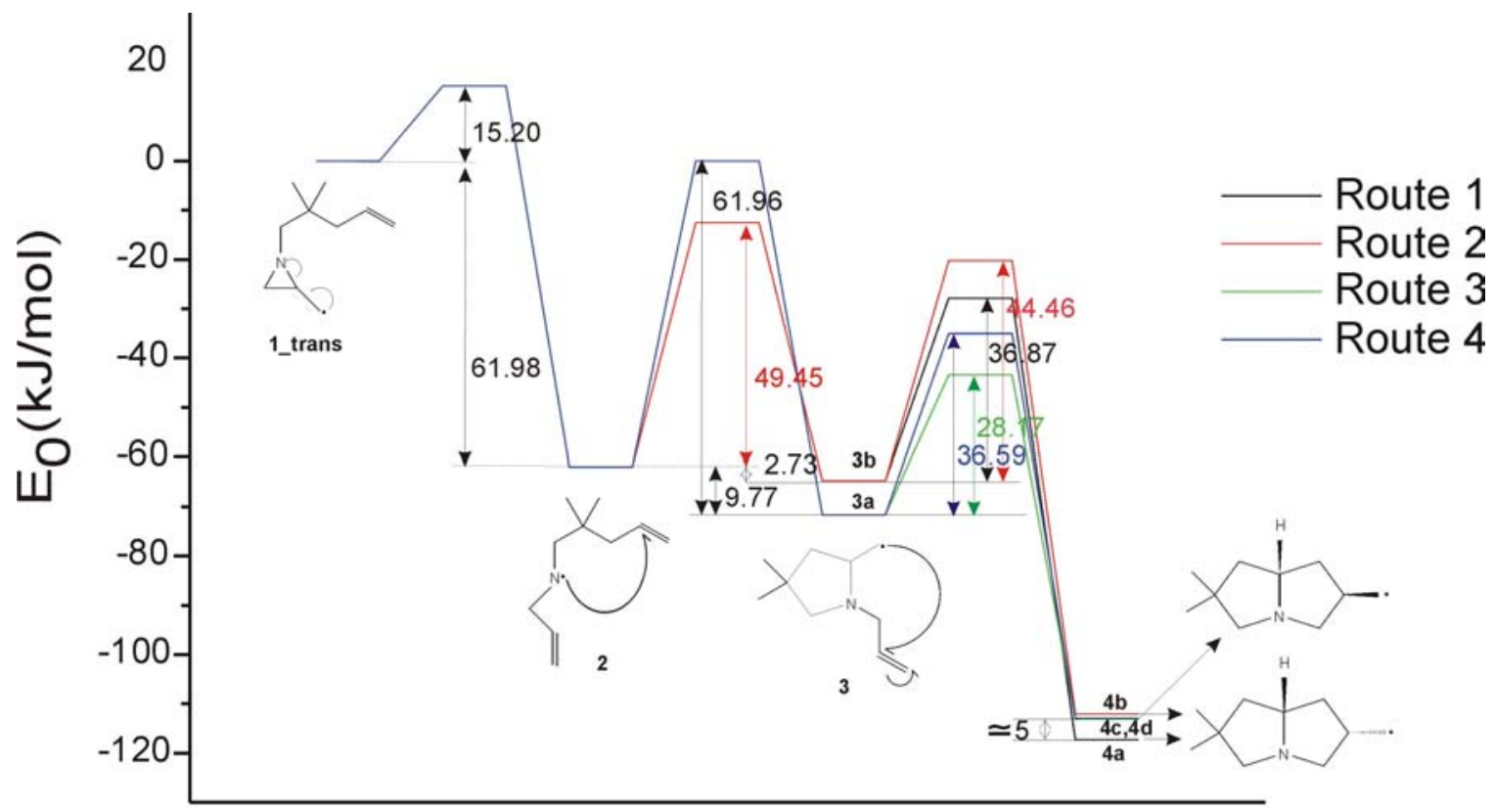

Reaction coordinate 
2. Z-matrices of all optimized structures at the B3LYP/6-311G** level of theory

C

C $\quad 1 \quad \mathrm{CC} 2$

c $2 \mathrm{cc} 3$

n 3 nc4

C $4 \mathrm{cn} 5$

$5 \mathrm{cc} 6$

$6 \mathrm{CC} 7$

$6 \mathrm{CC} 8$

$8 \mathrm{CC} 9$

$9 \mathrm{ccl}$

$6 \operatorname{ccl} 1$

10 hcl2

$10 \mathrm{hcl} 3$

9 hc14

8 hc15

8 hcl 6

11 hel 7

11 hc18

11 hel 9

7 hc20

7 hc21

7 hc22

5 hс23

5 hc24

3 hc25

3 hc26

2 hc2 7

1 hc28

h 1 hc 29

$1 \mathrm{CCC} 3$

$2 \operatorname{ncc} 4$

3 enc 5

$4 \operatorname{ccn} 6$

$5 \operatorname{ccc} 7$

$5 \operatorname{ccc} 8$

$6 \operatorname{ccc} 9$

$8 \operatorname{ccc} 10$

$5 \mathrm{cCC} 11$

$9 \mathrm{hcc} 12$

9 hccl3

8 hecl 4

$6 \mathrm{hcc} 15$

$6 \mathrm{hccl} 6$

$6 \mathrm{hccl} 7$

6 hccl 8

6 hcc19

6 hcc20

6 hcc2 1

6 hcc 22

4 hen23

4 hen 24

2 hcc25

2 hcc 26

4 hcn 27

2 hcc 28

2 hcc 29

$1 \operatorname{dih} 4$

2 dih5

3 dih 6

4 dih 7

4 dih8

5 dih 9

6 dih10

4 dihl1

8 dih12

8 dih13

6 dih14

5 dih 15

5 dih 16

5 dih 17

5 dih 18

5 dih19

5 dih 20

5 dih21

5 dih 22

2 dih 23

$2 \operatorname{dih} 24$

1 dih25

1 dih26

5 dih27

4 dih28

$\mathrm{CC} 2$

1. 452778

$\mathrm{cc} 3$

$\mathrm{CCC} 3$

1. 519384

123.180

1. 446715

nec 4

59.720

dih 4

105.373

cn 5

enc 5

dih 5

1.463238

116.230

108.994

1.545621

$\operatorname{ccn} 6$

dih 6

113.538

cC 7

$\mathrm{CCC} 7$

dih 7

$\mathrm{CC} 8$

$\operatorname{ccc} 8$

dih 8

cc 9

$\operatorname{ccc} 9$

dih 9

$-171.988$

1.538503

107.676

179.757

1.557930

108.077

$-61.261$

1. 501948

115.870

176.626

CC10

1.330906 


\begin{tabular}{|c|c|}
\hline $\operatorname{ccc} 10$ & 125.262 \\
\hline dih10 & 116.703 \\
\hline ccl1 & 1.539600 \\
\hline $\operatorname{ccc} 11$ & 110.185 \\
\hline dih11 & 60.394 \\
\hline hc12 & 1.084521 \\
\hline hcc12 & 121.736 \\
\hline $\operatorname{dih} 12$ & 178.688 \\
\hline 13 & 1.086292 \\
\hline $\operatorname{cc} 13$ & 121.578 \\
\hline his 13 & -0.794 \\
\hline 14 & 1.088332 \\
\hline hcc14 & 116.351 \\
\hline h1 4 & -64.655 \\
\hline 15 & 1.096368 \\
\hline$c 15$ & 108.321 \\
\hline h15 & -60.442 \\
\hline 16 & 1.095306 \\
\hline hcc16 & 107.112 \\
\hline $\ln 16$ & 54.307 \\
\hline 17 & 1.094834 \\
\hline $\operatorname{cc17}$ & 110.631 \\
\hline h17 & 55.121 \\
\hline hc18 & 1.092697 \\
\hline hсc18 & 111.063 \\
\hline h18 & 174.119 \\
\hline hc19 & 1.092581 \\
\hline c19 & 111.593 \\
\hline his & -65.046 \\
\hline hc20 & 1.094703 \\
\hline hcc20 & 111.059 \\
\hline $\ln 20$ & -62.619 \\
\hline 21 & 1.094891 \\
\hline$=21$ & 111.291 \\
\hline $\operatorname{dih} 21$ & 57.587 \\
\hline hc22 & 1.092381 \\
\hline hсc22 & 110.862 \\
\hline in 22 & 177.344 \\
\hline 23 & 1.104507 \\
\hline$=n 23$ & 111.459 \\
\hline dih23 & 22.933 \\
\hline hc24 & 1.096555 \\
\hline hen24 & 107.007 \\
\hline $\operatorname{din} 24$ & 139.465 \\
\hline hc25 & 1.085185 \\
\hline$=25$ & 119.414 \\
\hline dih25 & 0.382 \\
\hline hc26 & 1.089708 \\
\hline hсc26 & 116.427 \\
\hline h 26 & -145.618 \\
\hline 27 & 1.089570 \\
\hline $\operatorname{nn} 27$ & 115.656 \\
\hline $\operatorname{din} 27$ & -4.539 \\
\hline 28 & 1.082007 \\
\hline & 120.636 \\
\hline 28 & -162.029 \\
\hline & 1.08232 \\
\hline
\end{tabular}




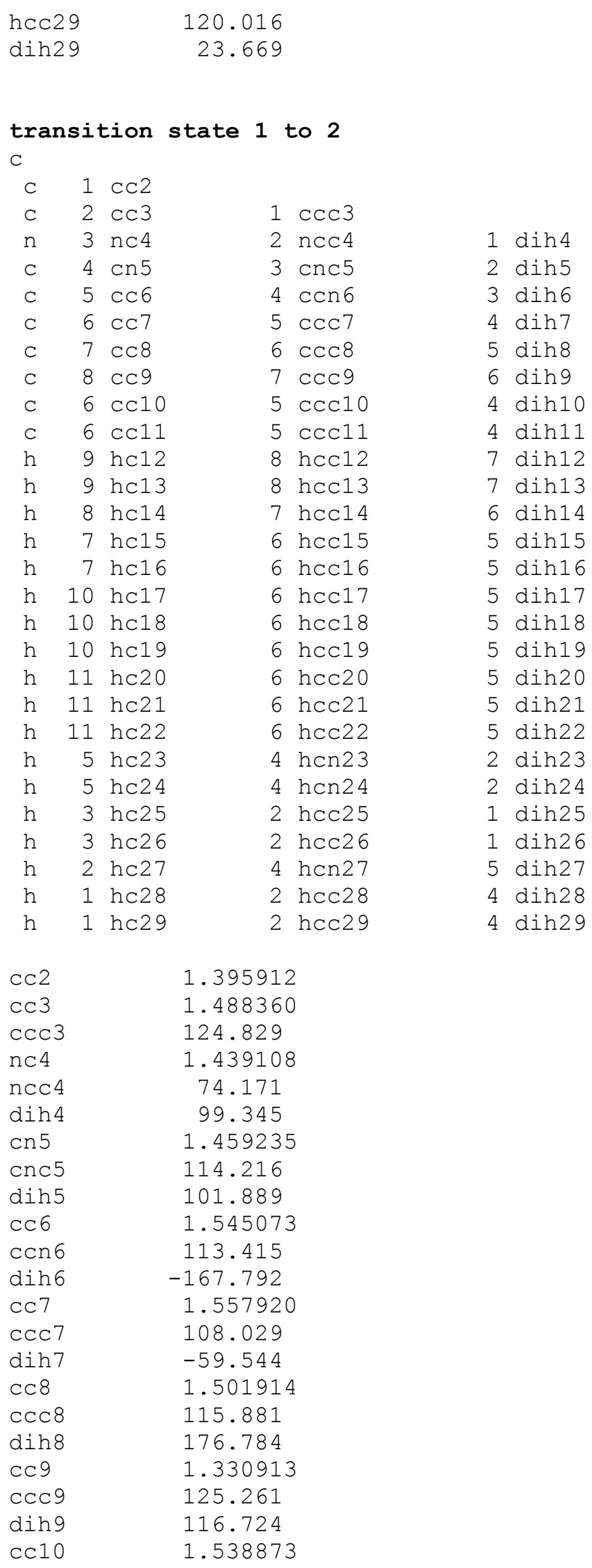




$\begin{array}{lc}\text { ccc10 } & 109.756 \\ \text { dih10 } & 61.825 \\ \text { cc11 } & 1.538128 \\ \text { ccc11 } & 107.933 \\ \text { dih11 } & -178.707 \\ \text { hc12 } & 1.084526 \\ \text { hcc12 } & 121.741 \\ \text { dih12 } & 178.690 \\ \text { hc13 } & 1.086312 \\ \text { hcc13 } & 121.576 \\ \text { dih13 } & -0.789 \\ \text { hc14 } & 1.088324 \\ \text { hcc14 } & 116.351 \\ \text { dih14 } & -64.629 \\ \text { hc15 } & 1.096540 \\ \text { hcc15 } & 108.318 \\ \text { dih15 } & -60.321 \\ \text { hc16 } & 1.095319 \\ \text { hcc16 } & 107.116 \\ \text { dih16 } & 54.465 \\ \text { hc17 } & 1.095080 \\ \text { hcc17 } & 110.576 \\ \text { dih17 } & 56.409 \\ \text { hc18 } & 1.092664 \\ \text { hcc18 } & 111.203 \\ \text { dih18 } & 175.435 \\ \text { hc19 } & 1.092111 \\ \text { hcc19 } & 111.228 \\ \text { dih19 } & -63.508 \\ \text { hc20 } & 1.094795 \\ \text { hcc20 } & 111.037 \\ \text { dih20 } & -62.021 \\ \text { hc21 } & 1.094928 \\ \text { hcc21 } & 111.282 \\ \text { dih21 } & 58.156 \\ \text { hc22 } & 1.092348 \\ \text { hcc22 } & 110.932 \\ \text { dih22 } & 177.924 \\ \text { hc23 } & 1.103050 \\ \text { hcn23 } & 112.640 \\ \text { dih23 } & 16.543 \\ \text { hc24 } & 1.102042 \\ \text { hcn24 } & 106.613 \\ \text { dih24 } & 132.403 \\ \text { hc25 } & 1.089316 \\ \text { hcc25 } & 116.139 \\ \text { dih25 } & -10.371 \\ \text { hc26 } & 1.093117 \\ \text { hcc26 } & 116.669 \\ \text { dih26 } & -146.840 \\ \text { hc27 } & 1.083310 \\ \text { hcn27 } & 106.691 \\ \text { dih27 } & 6.119 \\ \text { hc28 } & 1.082406 \\ \text { hcc28 } & 120.783 \\ \text { dih28 } & -121.425 \\ \text { hc29 } & 1.083060 \\ & \\ & \end{array}$




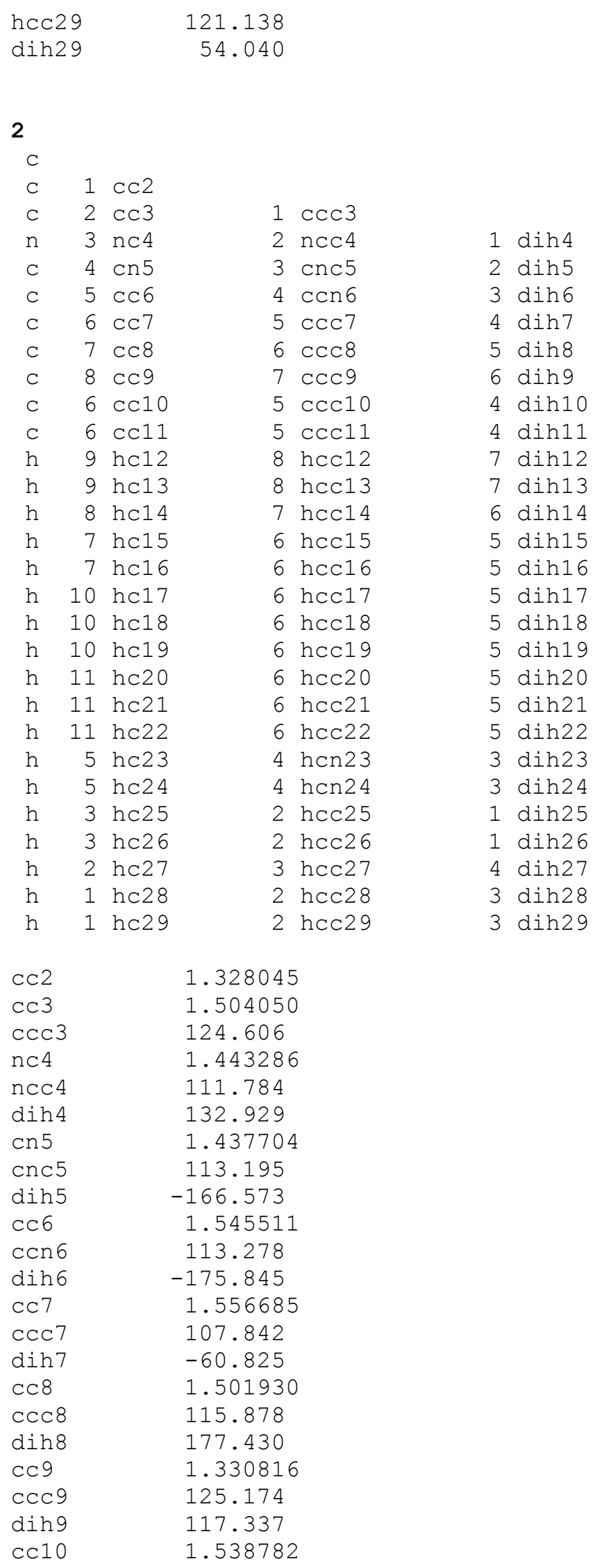




$\begin{array}{ll}\text { ccc10 } & 108.934 \\ \text { dih10 } & 60.086 \\ \text { cc11 } & 1.537185 \\ \text { ccc11 } & 108.279 \\ \text { dih11 } & 179.656 \\ \text { hc12 } & 1.084462 \\ \text { hcc12 } & 121.735 \\ \text { dih12 } & 178.791 \\ \text { hc13 } & 1.086300 \\ \text { hcc13 } & 121.575 \\ \text { dih13 } & -0.701 \\ \text { hc14 } & 1.088316 \\ \text { hcc14 } & 116.349 \\ \text { dih14 } & -63.901 \\ \text { hc15 } & 1.096718 \\ \text { hcc15 } & 108.424 \\ \text { dih15 } & -59.608 \\ \text { hc16 } & 1.096134 \\ \text { hcc16 } & 107.058 \\ \text { dih16 } & 55.228 \\ \text { hc17 } & 1.095059 \\ \text { hcc17 } & 110.468 \\ \text { dih17 } & 58.234 \\ \text { hc18 } & 1.092564 \\ \text { hcc18 } & 111.475 \\ \text { dih18 } & 177.517 \\ \text { hc19 } & 1.092255 \\ \text { hcc19 } & 110.812 \\ \text { dih19 } & -61.274 \\ \text { hc20 } & 1.094821 \\ \text { hcc20 } & 111.009 \\ \text { dih20 } & -60.954 \\ \text { hc21 } & 1.095139 \\ \text { hcc21 } & 111.282 \\ \text { dih21 } & 59.212 \\ \text { hc22 } & 1.092250 \\ \text { hcc22 } & 110.996 \\ \text { dih22 } & 178.980 \\ \text { hc23 } & 1.105571 \\ \text { hcn23 } & 109.951 \\ \text { dih23 } & -53.236 \\ \text { hc24 } & 1.105391 \\ \text { hcn24 } & 109.319 \\ \text { dih24 } & 61.830 \\ \text { hc25 } & 1.102589 \\ \text { hcc25 } & 110.559 \\ \text { dih25 } & 7.113 \\ \text { hc26 } & 1.107378 \\ \text { hcc26 } & 109.217 \\ \text { dih26 } & -108.517 \\ \text { hc27 } & 1.087061 \\ \text { hcc27 } & 115.007 \\ \text { dih27 } & -46.966 \\ \text { hc28 } & 1.083986 \\ \text { hcc28 } & 121.650 \\ \text { dih28 } & 179.161 \\ \text { hc29 } & 1.086248 \\ & \\ & \end{array}$




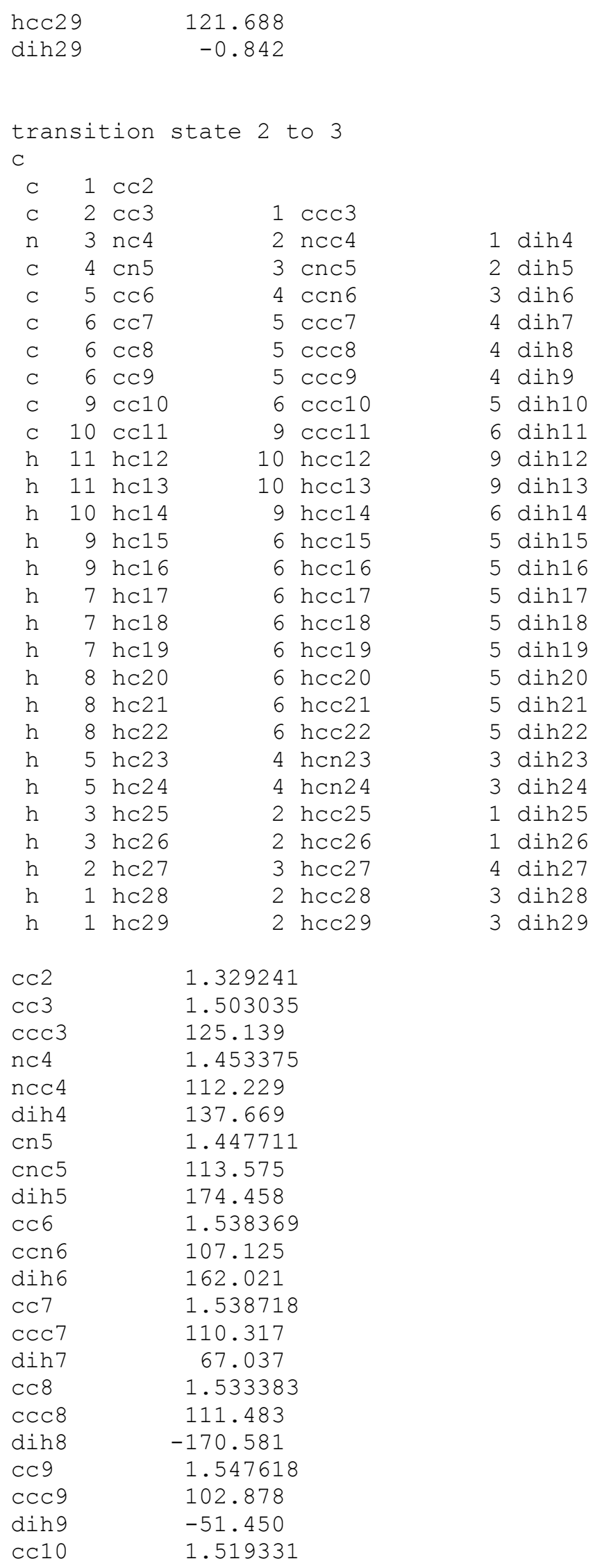




$\begin{array}{lc}\text { ccc10 } & 111.965 \\ \text { dih10 } & 40.621 \\ \text { cc11 } & 1.384086 \\ \text { ccc11 } & 119.909 \\ \text { dih11 } & -132.301 \\ \text { hc12 } & 1.082456 \\ \text { hcc12 } & 121.486 \\ \text { dih12 } & -166.753 \\ \text { hc13 } & 1.085336 \\ \text { hcc13 } & 120.946 \\ \text { dih13 } & 19.974 \\ \text { hc14 } & 1.083705 \\ \text { hcc14 } & 116.113 \\ \text { dih14 } & 78.383 \\ \text { hc15 } & 1.095689 \\ \text { hcc15 } & 110.564 \\ \text { dih15 } & 163.401 \\ \text { hc16 } & 1.095472 \\ \text { hcc16 } & 108.967 \\ \text { dih16 } & -79.496 \\ \text { hc17 } & 1.094808 \\ \text { hcc17 } & 110.647 \\ \text { dih17 } & 66.137 \\ \text { hc18 } & 1.094425 \\ \text { hcc18 } & 110.757 \\ \text { dih18 } & -174.498 \\ \text { hc19 } & 1.090969 \\ \text { hcc19 } & 111.198 \\ \text { dih19 } & -53.526 \\ \text { hc20 } & 1.094502 \\ \text { hcc20 } & 111.117 \\ \text { dih20 } & -63.792 \\ \text { hc21 } & 1.094448 \\ \text { hcc21 } & 111.367 \\ \text { dih21 } & 56.596 \\ \text { hc22 } & 1.094400 \\ \text { hcc22 } & 110.921 \\ \text { dih22 } & 176.678 \\ \text { hc23 } & 1.100915 \\ \text { hcn23 } & 108.846 \\ \text { dih23 } & -78.037 \\ \text { hc24 } & 1.105021 \\ \text { hcn24 } & 113.349 \\ \text { dih24 } & 40.655 \\ \text { hc25 } & 1.100995 \\ \text { hcc25 } & 110.670 \\ \text { dih25 } & 9.394 \\ \text { hc26 } & 1.103848 \\ \text { hcc26 } & 107.988 \\ \text { dih26 } & -105.808 \\ \text { hc27 } & 1.087829 \\ \text { hcc27 } & 114.762 \\ \text { dih27 } & -43.717 \\ \text { hc28 } & 1.084215 \\ \text { hcc28 } & 121.673 \\ \text { dih28 } & 177.798 \\ \text { hc29 } & 1.086195 \\ & \\ & \end{array}$




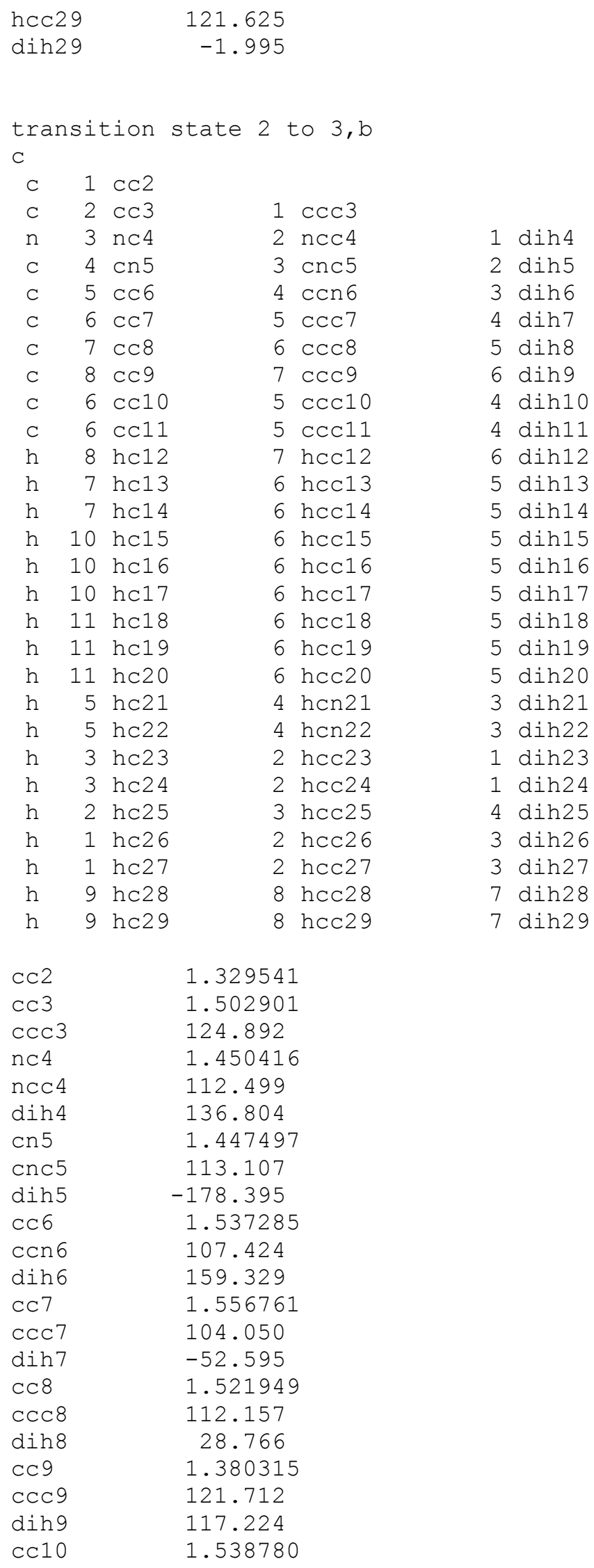




$\begin{array}{lc}\text { ccc10 } & 109.876 \\ \text { dih10 } & 65.267 \\ \text { cc11 } & 1.533296 \\ \text { ccc11 } & 111.355 \\ \text { dih11 } & -172.611 \\ \text { hc12 } & 1.087729 \\ \text { hcc12 } & 115.104 \\ \text { dih12 } & -94.077 \\ \text { hc13 } & 1.093738 \\ \text { hcc13 } & 110.065 \\ \text { dih13 } & 151.798 \\ \text { hc14 } & 1.095198 \\ \text { hcc14 } & 109.263 \\ \text { dih14 } & -91.399 \\ \text { hc15 } & 1.094888 \\ \text { hcc15 } & 110.726 \\ \text { dih15 } & 63.693 \\ \text { hc16 } & 1.094470 \\ \text { hcc16 } & 110.697 \\ \text { dih16 } & -176.829 \\ \text { hc17 } & 1.090810 \\ \text { hcc17 } & 110.787 \\ \text { dih17 } & -56.013 \\ \text { hc18 } & 1.094648 \\ \text { hcc18 } & 111.186 \\ \text { dih18 } & -64.208 \\ \text { hc19 } & 1.094557 \\ \text { hcc19 } & 111.381 \\ \text { dih19 } & 56.242 \\ \text { hc20 } & 1.094413 \\ \text { hcc20 } & 110.886 \\ \text { dih20 } & 176.271 \\ \text { hc21 } & 1.100886 \\ \text { hcn21 } & 108.937 \\ \text { dih21 } & -80.891 \\ \text { hc22 } & 1.106327 \\ \text { hcn22 } & 113.258 \\ \text { dih22 } & 37.886 \\ \text { hc23 } & 1.105381 \\ \text { hcc23 } & 110.324 \\ \text { dih23 } & 8.832 \\ \text { hc24 } & 1.104755 \\ \text { hcc24 } & 108.132 \\ \text { dih24 } & -106.133 \\ \text { hc25 } & 1.087326 \\ \text { hcc25 } & 114.835 \\ \text { dih25 } & -44.227 \\ \text { hc26 } & 1.084200 \\ \text { hcc26 } & 121.673 \\ \text { dih26 } & 178.096 \\ \text { hc27 } & 1.086317 \\ \text { hcc27 } & 121.668 \\ \text { dih27 } & -1.601 \\ \text { hc28 } & 1.082536 \\ \text { hcc28 } & 121.186 \\ \text { dih28 } & 168.182 \\ \text { hc29 } & 1.084753 \\ & \\ & \end{array}$


$\begin{array}{ll}\text { hec29 } & 121.012 \\ \operatorname{dih} 29 & -18.226\end{array}$

$3, a$

$\mathrm{C}$

C $\quad 1 \quad \mathrm{cC} 2$

n 2 nc3

c $3 \mathrm{cn} 4$

C $4 \mathrm{CC} 5$

c $2 \mathrm{cc} 6$

C $3 \mathrm{cn} 7$

C $\quad 7 \mathrm{CC} 8$

C $8 \mathrm{cc} 9$

C $5 \mathrm{CCl} 0$

C $5 \mathrm{ccl} 1$

h 6 hc12

h 6 hc13

h 2 hcl 4

h 1 hcl5

h 1 hcl6

h 10 hc17

h 10 hc18

h 10 hc19

h 11 hc20

h 11 hc21

h 11 hc22

h 4 hc23

h 4 hc24

h 7 hc25

h 7 hc26

h 8 hc27

h 9 hc28

h 9 hc2 9

$1 \mathrm{ncc} 3$

2 cnc4

$3 \operatorname{ccn} 5$

$3 \operatorname{ccn} 6$

$2 \operatorname{cnc} 7$

$3 \operatorname{ccn} 8$

$7 \operatorname{ccc} 9$

$4 \operatorname{ccc} 10$

$4 \mathrm{CCC} 11$

2 hccl2

2 hccl3

3 hen 14

2 hccl 5

$2 \operatorname{hcc} 16$

5 h ccl7

5 hecl 8

5 hec19

5 hcc 20

5 hсc21

5 hсc22

3 hen 23

3 hen24

3 hen 25

3 hen 26

7 hсc27

8 hcc2 8

8 hcc 29

1 dih4

2 dih5

4 dih 6

1 dih 7

2 dih8

3 dih9

3 dih10

3 dihl1

3 dih12

3 dih13

4 dih14

3 dih15

3 dih16

4 dih17

4 dih18

4 dih19

4 dih20

4 dih21

4 dih22

2 dih23

2 dih24

2 dih25

2 dih26

3 dih27

7 dih28

7 dih29

$\begin{array}{lc}\text { cc2 } & 1.565881 \\ \text { nc3 } & 1.482737 \\ \text { ncc } 3 & 101.924 \\ \text { cn4 } & 1.456187 \\ \text { cnc4 } & 106.664 \\ \text { dih4 } & -37.592 \\ \text { cc5 } & 1.545167 \\ \text { ccn5 } & 103.498 \\ \text { dih5 } & 45.442 \\ \text { cc6 } & 1.496600 \\ \text { ccn6 } & 114.895 \\ \text { dih6 } & 84.251 \\ \text { cn7 } & 1.458074 \\ \text { cnc7 } & 117.431 \\ \text { dih7 } & -170.640 \\ \text { cc } 8 & 1.504989 \\ \text { ccn8 } & 111.639 \\ \text { dih8 } & -67.548 \\ \text { cc9 } & 1.329166\end{array}$




\begin{tabular}{|c|c|}
\hline $\operatorname{ccc} 9$ & 124.972 \\
\hline dih 9 & 127.653 \\
\hline $\operatorname{cc} 10$ & 1.538577 \\
\hline $\operatorname{ccc} 10$ & 109.635 \\
\hline dih10 & 84.260 \\
\hline cc11 & 1.533559 \\
\hline $\operatorname{ccc} 11$ & 112.026 \\
\hline $\operatorname{dih} 11$ & -153.172 \\
\hline hc12 & 1.084461 \\
\hline$=12$ & 121.087 \\
\hline hin12 & -47.052 \\
\hline 13 & 1.082986 \\
\hline hcc13 & 121.045 \\
\hline h13 & 138.496 \\
\hline 14 & 1.093146 \\
\hline n 14 & 108.478 \\
\hline h1 4 & -154.109 \\
\hline hc15 & 1.092445 \\
\hline hcc15 & 110.325 \\
\hline dih15 & -105.163 \\
\hline 16 & 1.092021 \\
\hline c16 & 110.337 \\
\hline h16 & 136.567 \\
\hline hc17 & 1.094904 \\
\hline hсc17 & 111.037 \\
\hline dih17 & 67.598 \\
\hline 18 & 1.094437 \\
\hline c18 & 110.974 \\
\hline dih1 8 & -172.539 \\
\hline hc19 & 1.092420 \\
\hline c19 & 110.252 \\
\hline h19 & -52.466 \\
\hline 20 & 1.095037 \\
\hline 20 & 111.141 \\
\hline dih20 & -61.989 \\
\hline hc21 & 1.094113 \\
\hline hсc21 & 111.342 \\
\hline h21 & 58.281 \\
\hline 22 & 1.094393 \\
\hline 22 & 110.956 \\
\hline dih22 & 178.441 \\
\hline$h c 23$ & 1.094762 \\
\hline$n 23$ & 110.367 \\
\hline h23 & 166.044 \\
\hline 24 & 1.106646 \\
\hline$n 24$ & 113.076 \\
\hline $\operatorname{din} 24$ & -73.905 \\
\hline hc25 & 1.102864 \\
\hline$n 25$ & 113.196 \\
\hline h25 & 56.869 \\
\hline 26 & 1.097220 \\
\hline$n 26$ & 106.672 \\
\hline $\operatorname{dih} 26$ & 174.075 \\
\hline 27 & 1.088196 \\
\hline & 114.996 \\
\hline 27 & -52.652 \\
\hline & 1.084356 \\
\hline
\end{tabular}




\begin{tabular}{|c|c|c|c|c|c|c|}
\hline \multirow{2}{*}{\multicolumn{2}{|c|}{$\begin{array}{l}\text { hcc28 } \\
\text { dih28 }\end{array}$}} & & \multicolumn{2}{|c|}{121.699} & & \\
\hline & & & \multicolumn{2}{|l|}{178.594} & & \\
\hline \multicolumn{3}{|c|}{ hc29 } & \multicolumn{2}{|l|}{1.085927} & & \\
\hline \multicolumn{3}{|c|}{ hec29 } & 121.523 & & & \\
\hline \multicolumn{3}{|c|}{ dih29 } & -1.204 & & & \\
\hline \multicolumn{7}{|l|}{$3, b$} \\
\hline \multicolumn{7}{|l|}{ C } \\
\hline C & 1 & $\mathrm{cc} 2$ & & & & \\
\hline $\mathrm{C}$ & 2 & $\mathrm{cc} 3$ & 1 & $\operatorname{ccc} 3$ & & \\
\hline $\mathrm{n}$ & 3 & nc4 & 2 & nec4 & & dih4 \\
\hline C & 4 & cn5 & 3 & cnc 5 & 2 & dih 5 \\
\hline C & 3 & $\operatorname{cc} 6$ & 2 & $\operatorname{ccc} 6$ & 1 & $\operatorname{dih} 6$ \\
\hline $\mathrm{C}$ & 4 & $\mathrm{Cn} 7$ & 3 & $\mathrm{CnC} 7$ & 2 & $\operatorname{dih} 7$ \\
\hline C & 7 & $\mathrm{cc} 8$ & 4 & $\operatorname{ccn} 8$ & 3 & dih 8 \\
\hline C & 8 & $\mathrm{Cc} 9$ & 7 & $\operatorname{ccc} 9$ & 4 & dih 9 \\
\hline C & 1 & $\operatorname{cc} 10$ & 2 & $\operatorname{ccc} 10$ & 3 & dih 10 \\
\hline C & 1 & $\operatorname{cc} 11$ & 2 & $\operatorname{ccc} 11$ & 3 & dih11 \\
\hline $\mathrm{h}$ & 3 & hc12 & 2 & hccl2 & 1 & $\operatorname{dih} 12$ \\
\hline $\mathrm{h}$ & 2 & hc13 & 3 & hecl 3 & 4 & $\operatorname{dih} 13$ \\
\hline $\mathrm{h}$ & 2 & hc14 & 3 & hecl 4 & 4 & $\operatorname{dih} 14$ \\
\hline $\mathrm{h}$ & 10 & hc15 & 1 & hccl 5 & 2 & dih 15 \\
\hline $\mathrm{h}$ & 10 & hc1 6 & 1 & hccl 6 & 2 & $\operatorname{dih} 16$ \\
\hline $\mathrm{h}$ & 10 & hc1 7 & 1 & hccl 7 & 2 & $\operatorname{dih} 17$ \\
\hline $\mathrm{h}$ & 11 & hc18 & 1 & hccl 8 & 2 & $\operatorname{dih} 18$ \\
\hline $\mathrm{h}$ & 11 & he19 & 1 & hecl 9 & 2 & dih19 \\
\hline $\mathrm{h}$ & 11 & hc20 & 1 & hcc20 & 2 & $\operatorname{dih} 20$ \\
\hline $\mathrm{h}$ & 5 & hc21 & 4 & hen 21 & 3 & $\operatorname{dih} 21$ \\
\hline $\mathrm{h}$ & 5 & hc22 & 4 & hen 22 & 3 & $\operatorname{dih} 22$ \\
\hline $\mathrm{h}$ & 7 & hc23 & 4 & $\operatorname{hcn} 23$ & 3 & $\operatorname{dih} 23$ \\
\hline $\mathrm{h}$ & 7 & hc24 & 4 & $\operatorname{hen} 24$ & 3 & $\operatorname{din} 24$ \\
\hline $\mathrm{h}$ & 8 & hc25 & 7 & hcc25 & 4 & $\operatorname{dih} 25$ \\
\hline $\mathrm{h}$ & 9 & hc26 & 8 & hcc2 6 & 7 & $\operatorname{din} 26$ \\
\hline $\mathrm{h}$ & 9 & hc27 & 8 & hcc 27 & 7 & $\operatorname{dih} 27$ \\
\hline $\mathrm{h}$ & 6 & hc28 & 3 & hcc28 & 2 & $\operatorname{dih} 28$ \\
\hline $\mathrm{h}$ & 6 & hc29 & 3 & hcc2 9 & 2 & $\operatorname{din} 29$ \\
\hline $\mathrm{cc} 2$ & & & 1.561045 & & & \\
\hline $\mathrm{cc} 3$ & & & 1.563241 & & & \\
\hline $\mathrm{CCC} 3$ & & & 106.388 & & & \\
\hline $\mathrm{nc4}$ & & & 1.478671 & & & \\
\hline $\mathrm{ncc} 4$ & & & 102.752 & & & \\
\hline dih4 & & & 17.881 & & & \\
\hline cn 5 & & & 1.461091 & & & \\
\hline enc5 5 & & & 104.922 & & & \\
\hline dih5 & & & -38.993 & & & \\
\hline $\operatorname{cc} 6$ & & & 1.485360 & & & \\
\hline $\operatorname{ccc} 6$ & & & 113.699 & & & \\
\hline $\operatorname{dih} 6$ & & & 140.945 & & & \\
\hline $\mathrm{cn} 7$ & & & 1.460082 & & & \\
\hline $\mathrm{CnC} 7$ & & & 114.832 & & & \\
\hline $\operatorname{dih} 7$ & & & -163.792 & & & \\
\hline $\mathrm{CC} 8$ & & & 1.503490 & & & \\
\hline $\operatorname{ccn} 8$ & & & 113.877 & & & \\
\hline dih8 & & & -71.007 & & & \\
\hline $\operatorname{cc} 9$ & & & 1.330383 & & & \\
\hline $\operatorname{ccc} 9$ & & & 124.732 & & & \\
\hline
\end{tabular}




$\begin{array}{ll}\text { dih9 } & 135.143 \\ \text { cc10 } & 1.538275 \\ \text { ccc10 } & 110.362 \\ \text { dih10 } & -108.482 \\ \text { cc11 } & 1.533548 \\ \text { ccc11 } & 112.416 \\ \text { dih11 } & 128.146 \\ \text { hc12 } & 1.106569 \\ \text { hcc12 } & 108.166 \\ \text { dih12 } & -97.769 \\ \text { hc13 } & 1.092115 \\ \text { hcc13 } & 110.343 \\ \text { dih13 } & -102.367 \\ \text { hc14 } & 1.092326 \\ \text { hcc14 } & 110.004 \\ \text { dih14 } & 139.563 \\ \text { hc15 } & 1.094913 \\ \text { hcc15 } & 111.025 \\ \text { dih15 } & 179.243 \\ \text { hc16 } & 1.094483 \\ \text { hcc16 } & 110.959 \\ \text { dih16 } & -60.902 \\ \text { hc17 } & 1.092456 \\ \text { hcc17 } & 110.416 \\ \text { dih17 } & 59.181 \\ \text { hc18 } & 1.095165 \\ \text { hcc18 } & 111.135 \\ \text { dih18 } & -175.340 \\ \text { hc19 } & 1.094068 \\ \text { hcc19 } & 111.318 \\ \text { dih19 } & -55.114 \\ \text { hc20 } & 1.094313 \\ \text { hcc20 } & 110.946 \\ \text { dih20 } & 65.056 \\ \text { hc21 } & 1.094253 \\ \text { hcn21 } & 110.711 \\ \text { dih21 } & 167.466 \\ \text { hc22 } & 1.107958 \\ \text { hcn22 } & 111.377 \\ \text { dih22 } & -72.856 \\ \text { hc23 } & 1.107020 \\ \text { hcn23 } & 111.645 \\ \text { dih23 } & 53.871 \\ \text { hc24 } & 1.097453 \\ \text { hcn24 } & 106.651 \\ \text { dih24 } & 169.945 \\ \text { hc25 } & 1.087173 \\ \text { hcc25 } & 115.150 \\ \text { dih25 } & -48.068 \\ \text { hc26 } & 1.084015 \\ \text { hcc26 } & 121.635 \\ \text { dih26 } & 176.712 \\ \text { hc27 } & 1.085992 \\ \text { hcc27 } & 121.621 \\ \text { dih27 } & -3.592 \\ \text { hc28 } & 1.081813 \\ \text { hcc28 } & 121.594 \\ & \\ & \end{array}$




$\begin{array}{ll}\text { dih28 } & 105.971 \\ \text { hc29 } & 1.083574 \\ \text { hcc29 } & 119.357 \\ \text { dih29 } & -68.188\end{array}$

transition state $3 a$ to $4 a$

C

C $\quad 1 \quad \mathrm{cc} 2$

c $2 \mathrm{cc} 3$

n 3 nc4

c $4 \mathrm{cn} 5$

c $3 \mathrm{cc} 6$

C $\quad 4 \quad \mathrm{cn} 7$

C $\quad 7 \mathrm{cc} 8$

c $8 \mathrm{cc} 9$

C $1 \mathrm{cc} 10$

C $1 \mathrm{ccl} 1$

h 6 hc12

h 6 hc13

h 3 hcl 4

h 2 hcl5

h 2 hc16

h 10 hc17

h 10 hc18

h 10 hc19

h 11 hc20

h 11 hc21

h 11 hc22

h 5 hc23

h 5 hc2 4

h 7 hc25

h 7 hc26

h 8 hc27

h 9 hc28

h 9 hc2 9

119.357

68.188

$\mathrm{CC} 2$

cc 3

ccc 3

nc 4

$\operatorname{ncc} 4$

dih 4

cn 5

cnc5

dih5

cc 6

$\operatorname{ccc} 6$

dih 6

cn 7

cnc 7

dih 7

$\mathrm{cc} 8$

$\operatorname{ccn} 8$

dih8

cc 9

$\operatorname{ccc} 9$

\begin{tabular}{|c|c|c|}
\hline $1 \operatorname{ccc} 3$ & & \\
\hline $2 \operatorname{ncc} 4$ & 1 & dih 4 \\
\hline 3 cnc5 & 2 & dih 5 \\
\hline $2 \operatorname{ccc} 6$ & 1 & $\operatorname{dih} 6$ \\
\hline $3 \mathrm{cnc} 7$ & 2 & dih 7 \\
\hline $4 \operatorname{ccn} 8$ & 3 & dih 8 \\
\hline $7 \operatorname{ccc} 9$ & 4 & $\operatorname{din} 9$ \\
\hline $2 \operatorname{ccc} 10$ & 3 & dih10 \\
\hline $2 \operatorname{ccc} 11$ & 3 & dih11 \\
\hline $3 \mathrm{hcc} 12$ & 2 & dih12 \\
\hline $3 \mathrm{hcc} 13$ & 2 & dih13 \\
\hline $2 \operatorname{hccl} 4$ & 1 & $\operatorname{dih} 14$ \\
\hline $3 \mathrm{hcc} 15$ & 4 & dih15 \\
\hline $3 \mathrm{hcc} 16$ & 4 & dih16 \\
\hline $1 \operatorname{hcc} 17$ & 2 & $\operatorname{dih} 17$ \\
\hline $1 \mathrm{hcc} 18$ & 2 & dih18 \\
\hline 1 hccl9 & 2 & dih19 \\
\hline 1 hсc 20 & 2 & dih 20 \\
\hline 1 hсc 21 & 2 & $\operatorname{dih} 21$ \\
\hline 1 hсc 22 & 2 & dih 22 \\
\hline $4 \mathrm{hcn} 23$ & 3 & $\operatorname{dih} 23$ \\
\hline 4 hсn24 & 3 & dih24 \\
\hline 4 hcn25 & 3 & $\operatorname{dih} 25$ \\
\hline 4 hсn26 & 3 & dih26 \\
\hline 7 hсc 27 & 4 & $\operatorname{dih} 27$ \\
\hline 8 hсc28 & 7 & dih28 \\
\hline 8 hсc29 & 7 & dih29 \\
\hline
\end{tabular}

1.553154

1.542959

104.757

1.489907

104.542

$-35.227$

1. 468481

104.770

38.018

1.494814

116.351

$-158.305$

1.464706

110.519

$-85.888$

1.506659

110.298

$-55.973$

1.362479

123.005 


$\begin{array}{lc}\text { dih9 } & 150.510 \\ \text { cc10 } & 1.538397 \\ \text { ccc10 } & 110.925 \\ \text { dih10 } & -99.142 \\ \text { cc11 } & 1.537125 \\ \text { ccc11 } & 111.839 \\ \text { dih11 } & 138.571 \\ \text { hc12 } & 1.083524 \\ \text { hcc12 } & 119.005 \\ \text { dih12 } & -0.969 \\ \text { hc13 } & 1.083850 \\ \text { hcc13 } & 118.836 \\ \text { dih13 } & -150.668 \\ \text { hc14 } & 1.099645 \\ \text { hcc14 } & 108.672 \\ \text { dih14 } & 77.590 \\ \text { hc15 } & 1.093331 \\ \text { hcc15 } & 112.402 \\ \text { dih15 } & -157.585 \\ \text { hc16 } & 1.095028 \\ \text { hcc16 } & 109.515 \\ \text { dih16 } & 83.447 \\ \text { hc17 } & 1.094975 \\ \text { hcc17 } & 111.030 \\ \text { dih17 } & 173.116 \\ \text { hc18 } & 1.094347 \\ \text { hcc18 } & 111.075 \\ \text { dih18 } & -67.015 \\ \text { hc19 } & 1.094300 \\ \text { hcc19 } & 111.345 \\ \text { dih19 } & 53.235 \\ \text { hc20 } & 1.094903 \\ \text { hcc20 } & 111.071 \\ \text { dih20 } & -178.356 \\ \text { hc21 } & 1.093751 \\ \text { hcc21 } & 111.414 \\ \text { dih21 } & -58.151 \\ \text { hc22 } & 1.094947 \\ \text { hcc22 } & 111.075 \\ \text { dih22 } & 61.996 \\ \text { hc23 } & 1.095654 \\ \text { hcn23 } & 108.946 \\ \text { dih23 } & 92.904 \\ \text { hc24 } & 1.092868 \\ \text { hcn24 } & 110.233 \\ \text { dih24 } & -149.851 \\ \text { hc25 } & 1.104242 \\ \text { hcn25 } & 114.205 \\ \text { dih25 } & 67.033 \\ \text { hc26 } & 1.096230 \\ \text { hcn26 } & 107.385 \\ \text { dih26 } & -174.716 \\ \text { hc27 } & 1.086432 \\ \text { hcc27 } & 114.628 \\ \text { dih27 } & -51.782 \\ \text { hc28 } & 1.083337 \\ \text { hcc28 } & 121.464 \\ & \\ & \end{array}$




\begin{tabular}{|c|c|c|c|c|}
\hline dih & & 168.639 & & \\
\hline hc2 & & 1.085272 & & \\
\hline $\mathrm{hCC}$ & & 121.375 & & \\
\hline dih & & -15.735 & & \\
\hline $\begin{array}{l}\text { trai } \\
\mathrm{n}\end{array}$ & sition & state $3 a$ & to $4 \mathrm{~b}$ & \\
\hline $\mathrm{C}$ & $1 \mathrm{cn} 2$ & & & \\
\hline $\mathrm{C}$ & $2 \mathrm{cc} 3$ & 1 & $\operatorname{ccn} 3$ & \\
\hline C & $3 \mathrm{cc} 4$ & 2 & $\mathrm{CCC} 4$ & 1 dih4 \\
\hline $\mathrm{C}$ & $4 \mathrm{CC} 5$ & 3 & $\operatorname{ccc} 5$ & $2 \operatorname{dih} 5$ \\
\hline C & $3 \operatorname{cc} 6$ & 2 & $\operatorname{ccc} 6$ & $1 \operatorname{dih} 6$ \\
\hline C & $3 \mathrm{CC} 7$ & 2 & $\mathrm{CCC} 7$ & $1 \operatorname{dih} 7$ \\
\hline C & $5 \mathrm{cc} 8$ & 4 & $\operatorname{ccc} 8$ & 3 dih 8 \\
\hline C & 1 cn 9 & 2 & $\operatorname{cnc} 9$ & 3 dih 9 \\
\hline C & $9 \mathrm{cc} 10$ & 1 & $\operatorname{ccn} 10$ & $2 \operatorname{dih} 10$ \\
\hline C & $10 \mathrm{ccl} 1$ & 9 & $\operatorname{ccc} 11$ & $1 \operatorname{dih} 11$ \\
\hline $\mathrm{h}$ & 8 hc12 & 5 & hccl 12 & $4 \operatorname{dih} 12$ \\
\hline $\mathrm{h}$ & $8 \mathrm{hcl} 3$ & 5 & hecl 3 & $4 \operatorname{dih} 13$ \\
\hline $\mathrm{h}$ & 5 hcl 4 & 4 & hccl 4 & 3 dih14 \\
\hline $\mathrm{h}$ & 4 hcl 5 & 3 & hecl 5 & $2 \operatorname{dih} 15$ \\
\hline $\mathrm{h}$ & $4 \mathrm{hcl} 6$ & 3 & hccl 6 & $2 \operatorname{dih} 16$ \\
\hline $\mathrm{h}$ & 6 hcl 7 & 3 & hccl 7 & $2 \operatorname{dih} 17$ \\
\hline $\mathrm{h}$ & 6 hc18 & 3 & hccl 8 & $2 \operatorname{dih} 18$ \\
\hline $\mathrm{h}$ & 6 hcl 9 & 3 & hccl 9 & 2 dih19 \\
\hline $\mathrm{h}$ & 7 hc20 & 3 & hcc20 & $2 \operatorname{dih} 20$ \\
\hline $\mathrm{h}$ & 7 hс21 & 3 & hcc21 & $2 \operatorname{dih} 21$ \\
\hline $\mathrm{h}$ & 7 hc22 & 3 & hcc22 & $2 \operatorname{dih} 22$ \\
\hline $\mathrm{h}$ & 2 hс23 & 3 & hcc2 3 & 4 dih23 \\
\hline $\mathrm{h}$ & 2 hс24 & 3 & hcc2 4 & 4 dih24 \\
\hline $\mathrm{h}$ & 9 hc25 & 1 & hen 25 & 2 dih 25 \\
\hline $\mathrm{h}$ & 9 hс26 & 1 & hen 26 & $2 \operatorname{dih} 26$ \\
\hline $\mathrm{h}$ & 10 hc27 & 9 & hcc2 7 & $1 \operatorname{dih} 27$ \\
\hline $\mathrm{h}$ & 11 hс28 & 10 & hcc28 & 9 dih28 \\
\hline $\mathrm{h}$ & 11 hс29 & 10 & hсc 29 & 9 dih29 \\
\hline $\mathrm{cn} 2$ & & 1.468474 & & \\
\hline $\mathrm{Cc} 3$ & & 1.576188 & & \\
\hline $\mathrm{CCn}$ & & 109.075 & & \\
\hline $\mathrm{CC} 4$ & & 1.552759 & & \\
\hline $\mathrm{CCC}$ & & 102.626 & & \\
\hline dih & & 4.037 & & \\
\hline $\mathrm{CC} 5$ & & 1.545613 & & \\
\hline $\mathrm{CCC}$ & & 105.197 & & \\
\hline dih & & 18.155 & & \\
\hline $\mathrm{cc} 6$ & & 1.538248 & & \\
\hline $\mathrm{CCC}$ & & 110.201 & & \\
\hline dih & & 122.271 & & \\
\hline $\mathrm{CC} 7$ & & 1.536848 & & \\
\hline $\mathrm{CCC}^{-}$ & & 111.823 & & \\
\hline dih & & -115.882 & & \\
\hline $\mathrm{CC} 8$ & & 1.496164 & & \\
\hline $\mathrm{CCC}$ & & 115.538 & & \\
\hline dih & & -156.828 & & \\
\hline $\operatorname{cn} 9$ & & 1.466486 & & \\
\hline $\mathrm{CnC}$ & & 115.078 & & \\
\hline
\end{tabular}




$\begin{array}{lc}\text { dih9 } & 95.428 \\ \text { cc10 } & 1.516343 \\ \text { ccn10 } & 109.520 \\ \text { dih10 } & -178.084 \\ \text { cc11 } & 1.361769 \\ \text { ccc11 } & 122.082 \\ \text { dih11 } & -66.026 \\ \text { hc12 } & 1.085395 \\ \text { hcc12 } & 118.728 \\ \text { dih12 } & 6.585 \\ \text { hc13 } & 1.082305 \\ \text { hcc13 } & 119.186 \\ \text { dih13 } & -143.464 \\ \text { hc14 } & 1.097918 \\ \text { hcc14 } & 108.933 \\ \text { dih14 } & 79.053 \\ \text { hc15 } & 1.093254 \\ \text { hcc15 } & 112.443 \\ \text { dih15 } & 140.634 \\ \text { hc16 } & 1.095067 \\ \text { hcc16 } & 110.486 \\ \text { dih16 } & -99.793 \\ \text { hc17 } & 1.095007 \\ \text { hcc17 } & 111.055 \\ \text { dih17 } & 60.350 \\ \text { hc18 } & 1.094335 \\ \text { hcc18 } & 111.061 \\ \text { dih18 } & -179.774 \\ \text { hc19 } & 1.094337 \\ \text { hcc19 } & 111.334 \\ \text { dih19 } & -59.502 \\ \text { hc20 } & 1.094995 \\ \text { hcc20 } & 111.100 \\ \text { dih20 } & -64.068 \\ \text { hc21 } & 1.093785 \\ \text { hcc21 } & 111.353 \\ \text { dih21 } & 56.075 \\ \text { hc22 } & 1.094972 \\ \text { hcc22 } & 111.091 \\ \text { dih22 } & 176.252 \\ \text { hc23 } & 1.095774 \\ \text { hcc23 } & 109.380 \\ \text { dih23 } & -114.859 \\ \text { hc24 } & 1.092847 \\ \text { hcc24 } & 112.194 \\ \text { dih24 } & 126.501 \\ \text { hc25 } & 1.103553 \\ \text { hcn25 } & 113.612 \\ \text { dih25 } & -56.041 \\ \text { hc26 } & 1.094321 \\ \text { hcn26 } & 107.765 \\ \text { dih26 } & 62.167 \\ \text { hc27 } & 1.085859 \\ \text { hcc27 } & 116.620 \\ \text { dih27 } & 133.745 \\ \text { hc28 } & 1.083751 \\ \text { hcc28 } & 121.399 \\ & \\ & \end{array}$




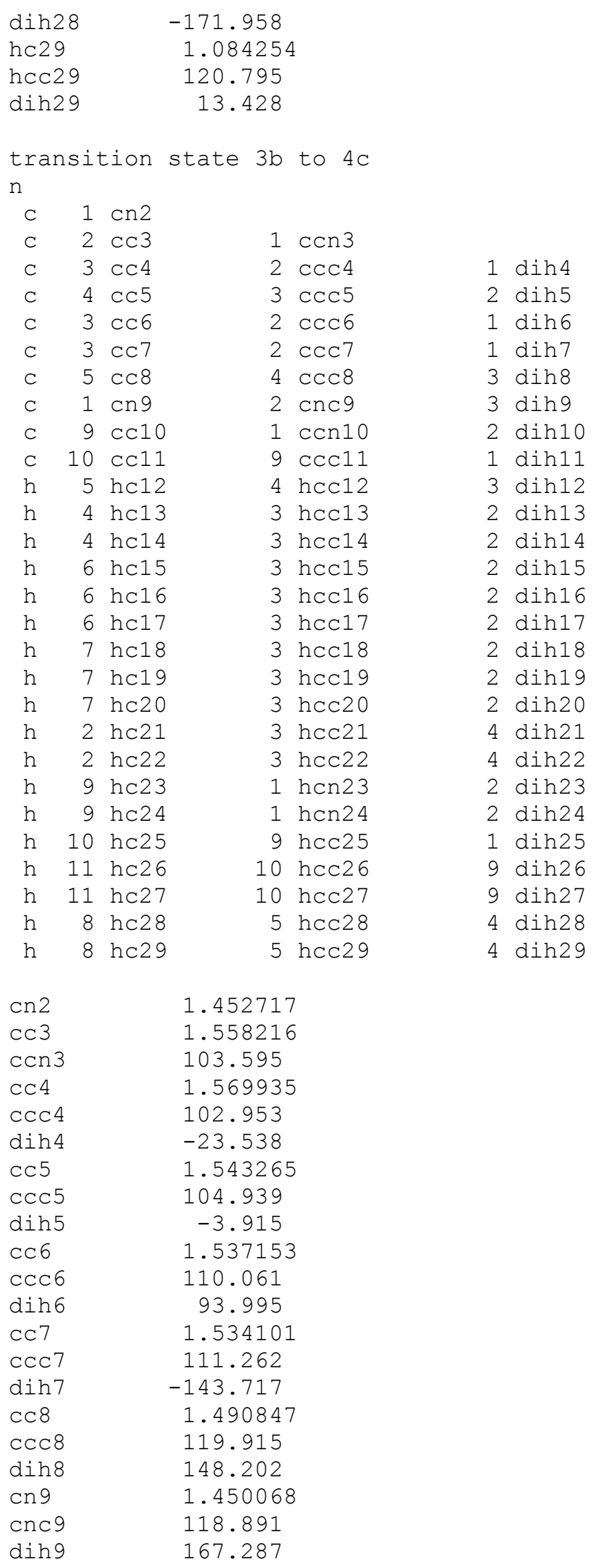




$\begin{array}{lc}\text { cc10 } & 1.516654 \\ \text { ccn10 } & 109.274 \\ \text { dih10 } & -173.786 \\ \text { cc11 } & 1.370255 \\ \text { ccc11 } & 121.873 \\ \text { dih11 } & 140.851 \\ \text { hc12 } & 1.109359 \\ \text { hcc12 } & 108.638 \\ \text { dih12 } & -86.169 \\ \text { hc13 } & 1.092742 \\ \text { hcc13 } & 110.410 \\ \text { dih13 } & 114.294 \\ \text { hc14 } & 1.092384 \\ \text { hcc14 } & 112.374 \\ \text { dih14 } & -125.492 \\ \text { hc15 } & 1.094939 \\ \text { hcc15 } & 111.155 \\ \text { dih15 } & 67.454 \\ \text { hc16 } & 1.094620 \\ \text { hcc16 } & 111.031 \\ \text { dih16 } & -172.545 \\ \text { hc17 } & 1.093108 \\ \text { hcc17 } & 110.367 \\ \text { dih17 } & -52.649 \\ \text { hc18 } & 1.095186 \\ \text { hcc18 } & 111.094 \\ \text { dih18 } & -59.631 \\ \text { hc19 } & 1.094058 \\ \text { hcc19 } & 111.328 \\ \text { dih19 } & 60.458 \\ \text { hc20 } & 1.094446 \\ \text { hcc20 } & 110.965 \\ \text { dih20 } & -179.324 \\ \text { hc21 } & 1.094117 \\ \text { hcc21 } & 112.496 \\ \text { dih21 } & -144.042 \\ \text { hc22 } & 1.107684 \\ \text { hcc22 } & 110.109 \\ \text { dih22 } & 95.799 \\ \text { hc23 } & 1.107815 \\ \text { hcn23 } & 111.826 \\ \text { dih23 } & -52.722 \\ \text { hc24 } & 1.095890 \\ \text { hcn24 } & 109.394 \\ \text { dih24 } & 66.114 \\ \text { hc25 } & 1.086040 \\ \text { hcc25 } & 114.790 \\ \text { dih25 } & -65.606 \\ \text { hc26 } & 1.083444 \\ \text { hcc26 } & 121.427 \\ \text { dih26 } & 167.834 \\ \text { hc27 } & 1.084996 \\ \text { hcc27 } & 121.293 \\ \text { dih27 } & -17.429 \\ \text { hc28 } & 1.083581 \\ \text { hcc28 } & 119.307 \\ \text { dih28 } & 102.295 \\ & \end{array}$




\begin{tabular}{|c|c|c|c|c|}
\hline hc2 & & 1.086193 & & \\
\hline $\mathrm{hCC}$ & & 117.229 & & \\
\hline dih & & -45.521 & & \\
\hline $\begin{array}{l}\text { tra } \\
n\end{array}$ & isition & state $3 b$ & to $4 d$ & \\
\hline C & $1 \mathrm{cn} 2$ & & & \\
\hline C & $2 \operatorname{cc} 3$ & 1 & $\operatorname{ccn} 3$ & \\
\hline C & $3 \mathrm{cc} 4$ & 2 & $\mathrm{CCC} 4$ & 1 dih4 \\
\hline C & $4 \mathrm{CC} 5$ & 3 & $\operatorname{ccc} 5$ & 2 dih 5 \\
\hline C & $3 \operatorname{cc} 6$ & 2 & $\operatorname{ccc} 6$ & 1 dih 6 \\
\hline C & $3 \mathrm{cC} 7$ & 2 & $\operatorname{ccc} 7$ & $1 \operatorname{dih} 7$ \\
\hline C & $5 \operatorname{cc} 8$ & 4 & $\operatorname{ccc} 8$ & 3 dih8 \\
\hline $\mathrm{C}$ & $1 \mathrm{cn} 9$ & 2 & cnc 9 & 3 dih 9 \\
\hline C & $9 \mathrm{cc} 10$ & 1 & $\operatorname{ccn} 10$ & $2 \operatorname{dih} 10$ \\
\hline C & $10 \mathrm{cc} 11$ & 9 & $\operatorname{ccc} 11$ & $1 \operatorname{dih} 11$ \\
\hline $\mathrm{h}$ & 10 hc12 & 9 & hccl2 & $1 \operatorname{dih} 12$ \\
\hline $\mathrm{h}$ & 5 hс13 & 4 & hecl 3 & 3 dih13 \\
\hline $\mathrm{h}$ & 4 hcl 4 & 3 & $\mathrm{hcc} 14$ & 2 dih14 \\
\hline h & 4 hcl 5 & 3 & hccl 5 & 2 dih15 \\
\hline $\mathrm{h}$ & $6 \mathrm{hcl} 6$ & 3 & hccl 6 & $2 \operatorname{dih} 16$ \\
\hline $\mathrm{h}$ & 6 hcl 7 & 3 & hccl 7 & $2 \operatorname{dih} 17$ \\
\hline $\mathrm{h}$ & 6 hcl 8 & 3 & hccl 8 & 2 dih18 \\
\hline h & 7 hcl 9 & 3 & hccl 9 & $2 \operatorname{dih} 19$ \\
\hline $\mathrm{h}$ & 7 hc20 & 3 & hcc20 & $2 \operatorname{dih} 20$ \\
\hline $\mathrm{h}$ & 7 hс21 & 3 & hcc21 & $2 \operatorname{dih} 21$ \\
\hline $\mathrm{h}$ & 2 hс22 & 3 & hcc 22 & 4 dih22 \\
\hline $\mathrm{h}$ & 2 hс23 & 3 & hcc23 & 4 dih23 \\
\hline $\mathrm{h}$ & 9 hс24 & 1 & $\operatorname{hcn} 24$ & $2 \operatorname{dih} 24$ \\
\hline $\mathrm{h}$ & 9 hc25 & 1 & hen 25 & $2 \operatorname{dih} 25$ \\
\hline $\mathrm{h}$ & 8 hc26 & 5 & hcc2 6 & 4 dih26 \\
\hline $\mathrm{h}$ & 8 hс27 & 5 & hcc 27 & 4 dih27 \\
\hline $\mathrm{h}$ & 11 hс28 & 10 & hcc28 & 9 dih28 \\
\hline $\mathrm{h}$ & 11 hc29 & 10 & hcc2 9 & $9 \operatorname{din} 29$ \\
\hline $\mathrm{cn} 2$ & & 1.452709 & & \\
\hline $\mathrm{CC} 3$ & & 1.558001 & & \\
\hline $\mathrm{CCn}$ & & 103.632 & & \\
\hline $\mathrm{CC} 4$ & & 1.569456 & & \\
\hline $\mathrm{CCC}$ & & 102.889 & & \\
\hline dih & & -23.325 & & \\
\hline $\operatorname{cc} 5$ & & 1.544237 & & \\
\hline $\mathrm{CCC}$ & & 104.994 & & \\
\hline dih & & -4.098 & & \\
\hline $\operatorname{cc} 6$ & & 1.536980 & & \\
\hline $\mathrm{CCC}$ & & 110.106 & & \\
\hline dih & & 94.190 & & \\
\hline $\mathrm{CC} 7$ & & 1.534308 & & \\
\hline $\mathrm{CCC}$ & & 111.280 & & \\
\hline dih & & -143.434 & & \\
\hline $\mathrm{cc} 8$ & & 1.490340 & & \\
\hline $\mathrm{CCC}$ & & 119.475 & & \\
\hline dih & & 148.077 & & \\
\hline $\operatorname{cn} 9$ & & 1.454011 & & \\
\hline $\mathrm{CnC}$ & & 118.390 & & \\
\hline dih & & 168.086 & & \\
\hline $\mathrm{CC} 1$ & & 1.526510 & & \\
\hline $\mathrm{CCn}$ & & 110.046 & & \\
\hline
\end{tabular}




$\begin{array}{lc}\text { dih10 } & -170.208 \\ \text { cc11 } & 1.368060 \\ \text { ccc11 } & 121.517 \\ \text { dih11 } & -94.295 \\ \text { hc12 } & 1.085895 \\ \text { hcc12 } & 116.108 \\ \text { dih12 } & 110.647 \\ \text { hc13 } & 1.109933 \\ \text { hcc13 } & 108.555 \\ \text { dih13 } & -86.287 \\ \text { hc14 } & 1.092697 \\ \text { hcc14 } & 110.453 \\ \text { dih14 } & 114.001 \\ \text { hc15 } & 1.092349 \\ \text { hcc15 } & 112.370 \\ \text { dih15 } & -125.732 \\ \text { hc16 } & 1.094904 \\ \text { hcc16 } & 111.177 \\ \text { dih16 } & 67.508 \\ \text { hc17 } & 1.094625 \\ \text { hcc17 } & 111.011 \\ \text { dih17 } & -172.462 \\ \text { hc18 } & 1.093100 \\ \text { hcc18 } & 110.313 \\ \text { dih18 } & -52.619 \\ \text { hc19 } & 1.095210 \\ \text { hcc19 } & 111.139 \\ \text { dih19 } & -59.794 \\ \text { hc20 } & 1.094067 \\ \text { hcc20 } & 111.326 \\ \text { dih20 } & 60.306 \\ \text { hc21 } & 1.094422 \\ \text { hcc21 } & 110.921 \\ \text { dih21 } & -179.497 \\ \text { hc22 } & 1.094220 \\ \text { hcc22 } & 112.547 \\ \text { dih22 } & -143.840 \\ \text { hc23 } & 1.107949 \\ \text { hcc23 } & 110.187 \\ \text { dih23 } & 96.005 \\ \text { hc24 } & 1.107557 \\ \text { hcn24 } & 111.166 \\ \text { dih24 } & -49.546 \\ \text { hc25 } & 1.093869 \\ \text { hcn25 } & 109.272 \\ \text { dih25 } & 68.668 \\ \text { hc26 } & 1.084736 \\ \text { hcc26 } & 119.555 \\ \text { dih26 } & 101.869 \\ \text { hc27 } & 1.084624 \\ \text { hcc27 } & 117.178 \\ \text { dih27 } & -46.746 \\ \text { hc28 } & 1.083765 \\ \text { hcc28 } & 121.479 \\ \text { dih28 } & -170.761 \\ \text { hc29 } & 1.084949 \\ \text { hcc29 } & 121.075 \\ & \\ & \end{array}$




\begin{tabular}{|c|c|c|c|}
\hline \multicolumn{4}{|l|}{$4, a$} \\
\hline \multicolumn{4}{|l|}{ C } \\
\hline C & $1 \mathrm{cc} 2$ & & \\
\hline $\mathrm{n}$ & $2 \mathrm{nc} 3$ & $1 \mathrm{ncc} 3$ & \\
\hline C & $3 \mathrm{cn} 4$ & 2 cnc4 & 1 dih4 \\
\hline C & $4 \mathrm{cc} 5$ & $3 \operatorname{ccn} 5$ & 2 dih5 \\
\hline C & $3 \operatorname{cn} 6$ & 2 cnc 6 & 1 dih 6 \\
\hline $\mathrm{C}$ & $6 \mathrm{cc} 7$ & $3 \operatorname{ccn} 7$ & $2 \operatorname{dih} 7$ \\
\hline C & $7 \operatorname{cc} 8$ & $6 \operatorname{ccc} 8$ & 3 dih 8 \\
\hline C & $7 \operatorname{cc} 9$ & $8 \operatorname{ccc} 9$ & $2 \operatorname{dih} 9$ \\
\hline C & $7 \operatorname{ccl} 0$ & $8 \operatorname{ccc} 10$ & $2 \operatorname{dih} 10$ \\
\hline C & $5 \operatorname{ccl} 1$ & $4 \operatorname{ccc} 11$ & 3 dih11 \\
\hline $\mathrm{h}$ & $1 \mathrm{hcl} 2$ & $2 \mathrm{hcc} 12$ & 8 dih 12 \\
\hline $\mathrm{h}$ & $1 \mathrm{hcl} 3$ & $2 \operatorname{hcc} 13$ & 8 dih13 \\
\hline $\mathrm{h}$ & $2 \mathrm{hcl} 4$ & 8 hcc14 & 7 dih14 \\
\hline $\mathrm{h}$ & 8 hcl 5 & 2 hсc15 & 3 dih15 \\
\hline $\mathrm{h}$ & 8 hc1 6 & $2 \operatorname{hcc} 16$ & $3 \operatorname{dih} 16$ \\
\hline $\mathrm{h}$ & 9 hcl 7 & 7 hcc17 & $8 \operatorname{dih} 17$ \\
\hline $\mathrm{h}$ & 9 hc18 & 7 hсc18 & 8 dih18 \\
\hline $\mathrm{h}$ & 9 hcl 9 & 7 hсc19 & 8 dih19 \\
\hline $\mathrm{h}$ & 10 hc20 & 7 hсc20 & 8 dih20 \\
\hline $\mathrm{h}$ & 10 hc21 & 7 hсc21 & 8 dih21 \\
\hline $\mathrm{h}$ & 10 hс22 & 7 hсc 22 & 8 dih22 \\
\hline $\mathrm{h}$ & 6 hc23 & $3 \operatorname{hcn} 23$ & $2 \operatorname{dih} 23$ \\
\hline $\mathrm{h}$ & 6 hс24 & $3 \operatorname{hcn} 24$ & $2 \operatorname{dih} 24$ \\
\hline $\mathrm{h}$ & 4 hc25 & $3 \operatorname{hcn} 25$ & $2 \operatorname{dih} 25$ \\
\hline $\mathrm{h}$ & $4 \mathrm{hc} 26$ & $3 \operatorname{hcn} 26$ & $2 \operatorname{dih} 26$ \\
\hline $\mathrm{h}$ & 5 hс27 & 4 hсc 27 & $3 \operatorname{dih} 27$ \\
\hline $\mathrm{h}$ & 11 hc28 & 5 hсc 28 & 4 dih28 \\
\hline $\mathrm{h}$ & 11 hс29 & 5 hсc 29 & $4 \operatorname{dih} 29$ \\
\hline $\mathrm{CC} 2$ & & 1.538199 & \\
\hline nc3 & & 1.486713 & \\
\hline $\mathrm{ncc} 3$ & & 105.178 & \\
\hline $\mathrm{cn} 4$ & & 1.464550 & \\
\hline Cnc4 & & 110.076 & \\
\hline dih4 & & -3.581 & \\
\hline cC5 & & 1.552372 & \\
\hline $\operatorname{ccn} 5$ & & 104.598 & \\
\hline dih5 & & -20.744 & \\
\hline $\operatorname{cn} 6$ & & 1.462449 & \\
\hline enc 6 & & 109.288 & \\
\hline $\operatorname{dih} 6$ & & 128.240 & \\
\hline $\mathrm{CC} 7$ & & 1.554583 & \\
\hline $\operatorname{ccn} 7$ & & 107.599 & \\
\hline $\operatorname{dih} 7$ & & -21.236 & \\
\hline $\mathrm{CC} 8$ & & 1.552186 & \\
\hline $\operatorname{ccc} 8$ & & 101.275 & \\
\hline
\end{tabular}




$\begin{array}{lc}\text { dih8 } & 30.168 \\ \text { cc9 } & 1.534030 \\ \text { ccc9 } & 112.063 \\ \text { dih9 } & -147.306 \\ \text { cc10 } & 1.540983 \\ \text { ccc10 } & 111.344 \\ \text { dih10 } & 90.127 \\ \text { cc11 } & 1.484501 \\ \text { ccc11 } & 114.562 \\ \text { dih11 } & 160.396 \\ \text { hc12 } & 1.095105 \\ \text { hcc12 } & 110.947 \\ \text { dih12 } & 26.962 \\ \text { hc13 } & 1.092427 \\ \text { hcc13 } & 112.964 \\ \text { dih13 } & -93.892 \\ \text { hc14 } & 1.097242 \\ \text { hcc14 } & 109.324 \\ \text { dih14 } & 134.658 \\ \text { hc15 } & 1.094135 \\ \text { hcc15 } & 109.661 \\ \text { dih15 } & -101.663 \\ \text { hc16 } & 1.093648 \\ \text { hcc16 } & 112.129 \\ \text { dih16 } & 139.581 \\ \text { hc17 } & 1.095020 \\ \text { hcc17 } & 111.127 \\ \text { dih17 } & 176.495 \\ \text { hc18 } & 1.094576 \\ \text { hcc18 } & 111.171 \\ \text { dih18 } & -63.857 \\ \text { hc19 } & 1.093983 \\ \text { hcc19 } & 111.182 \\ \text { dih19 } & 56.345 \\ \text { hc20 } & 1.094673 \\ \text { hcc20 } & 110.746 \\ \text { dih20 } & -177.697 \\ \text { hc21 } & 1.091567 \\ \text { hcc21 } & 112.222 \\ \text { dih21 } & -57.434 \\ \text { hc22 } & 1.094738 \\ \text { hcc22 } & 110.756 \\ \text { dih22 } & 62.869 \\ \text { hc23 } & 1.098537 \\ \text { hcn23 } & 110.013 \\ \text { dih23 } & 97.805 \\ \text { hc24 } & 1.094762 \\ \text { hcn24 } & 110.613 \\ \text { dih24 } & -144.315 \\ \text { hc25 } & 1.102847 \\ \text { hcn25 } & 112.689 \\ \text { dih25 } & 98.281 \\ \text { hc26 } & 1.094856 \\ \text { hcn26 } & 111.673 \\ \text { dih26 } & -140.953 \\ \text { hc27 } & 1.095413 \\ \text { hcc27 } & 107.589 \\ & \\ & \end{array}$




$\begin{array}{ll}\text { dih2 } & -77.411 \\ \text { hc28 } & 1.085093 \\ \text { hcc28 } & 120.040 \\ \text { dih28 } & -65.466 \\ \text { hc29 } & 1.082198 \\ \text { hcc29 } & 122.069 \\ \text { dih29 } & 112.170\end{array}$

\begin{tabular}{|c|c|c|c|}
\hline \multicolumn{4}{|l|}{$4, b$} \\
\hline \multicolumn{4}{|l|}{$\mathrm{C}$} \\
\hline c & $1 \mathrm{cc} 2$ & & \\
\hline $\mathrm{n}$ & $2 \mathrm{nc} 3$ & $1 \operatorname{ncc} 3$ & \\
\hline $\mathrm{C}$ & $3 \mathrm{cn} 4$ & $2 \mathrm{cnc} 4$ & $1 \operatorname{dih} 4$ \\
\hline $\mathrm{C}$ & $4 \mathrm{cC5}$ & $3 \operatorname{ccn} 5$ & $2 \operatorname{dih} 5$ \\
\hline C & $3 \mathrm{cn} 6$ & 2 cnc 6 & 1 dih 6 \\
\hline $\mathrm{C}$ & $6 \mathrm{cc} 7$ & $3 \operatorname{ccn} 7$ & $2 \operatorname{dih} 7$ \\
\hline $\mathrm{C}$ & $7 \mathrm{cc} 8$ & $6 \operatorname{ccc} 8$ & 3 dih 8 \\
\hline C & $7 \operatorname{cc} 9$ & $8 \operatorname{ccc} 9$ & 2 dih 9 \\
\hline $\mathrm{c}$ & $7 \mathrm{ccl} 10$ & $8 \operatorname{ccc} 10$ & $2 \operatorname{dih} 10$ \\
\hline $\mathrm{C}$ & $5 \mathrm{ccl} 1$ & $4 \operatorname{ccc} 11$ & 3 dih11 \\
\hline $\mathrm{h}$ & $1 \mathrm{hc} 12$ & $2 \mathrm{hcc} 12$ & 8 dih12 \\
\hline $\mathrm{h}$ & $1 \mathrm{hc13}$ & 2 hсc13 & 8 dih13 \\
\hline $\mathrm{h}$ & 2 hc14 & 8 hсc14 & 7 dih14 \\
\hline $\mathrm{h}$ & 8 hc15 & $2 \mathrm{hcc} 15$ & 3 dih15 \\
\hline $\mathrm{h}$ & 8 hс16 & $2 \mathrm{hcc} 16$ & 3 dih16 \\
\hline $\mathrm{h}$ & 9 hс17 & 7 hcc17 & 8 dih17 \\
\hline $\mathrm{h}$ & $9 \mathrm{hcl} 8$ & $7 \mathrm{hcc} 18$ & 8 dih18 \\
\hline $\mathrm{h}$ & 9 hс19 & 7 hec19 & 8 dih19 \\
\hline $\mathrm{h}$ & $10 \mathrm{hc} 20$ & $7 \mathrm{hcc} 20$ & 8 dih20 \\
\hline $\mathrm{h}$ & $10 \mathrm{hc} 21$ & $7 \mathrm{hcc} 21$ & 8 dih21 \\
\hline $\mathrm{h}$ & 10 hс22 & 7 hсc22 & 8 dih22 \\
\hline $\mathrm{h}$ & 6 hс23 & $3 \operatorname{hcn} 23$ & 2 dih23 \\
\hline $\mathrm{h}$ & $6 \mathrm{hc} 24$ & $3 \mathrm{hcn} 24$ & 2 dih24 \\
\hline $\mathrm{h}$ & 4 hс25 & $3 \operatorname{hcn} 25$ & 2 dih25 \\
\hline $\mathrm{h}$ & $4 \mathrm{hc26}$ & $3 \mathrm{hcn} 26$ & 2 dih26 \\
\hline $\mathrm{h}$ & 5 hс27 & $4 \mathrm{hcc} 27$ & 3 dih27 \\
\hline $\mathrm{h}$ & 11 hc28 & 5 hсc28 & $4 \operatorname{dih} 28$ \\
\hline $\mathrm{h}$ & 11 hc29 & 5 hсс29 & 4 dih29 \\
\hline $\mathrm{cc} 2$ & & 1.534044 & \\
\hline nc3 & & 1.477227 & \\
\hline ncc3 & & 104.666 & \\
\hline $\mathrm{cn} 4$ & & 1.472024 & \\
\hline $\mathrm{cnc} 4$ & & 109.595 & \\
\hline dih4 & & 16.571 & \\
\hline cc5 & & 1.553682 & \\
\hline c cn5 & & 106.850 & \\
\hline dih5 & & 7.561 & \\
\hline $\mathrm{cn} 6$ & & 1.458105 & \\
\hline $\mathrm{cnc} 6$ & & 107.321 & \\
\hline $\operatorname{dih} 6$ & & 147.917 & \\
\hline $\mathrm{CC} 7$ & & 1.562841 & \\
\hline $\operatorname{ccn} 7$ & & 108.434 & \\
\hline dih7 & & -29.857 & \\
\hline $\mathrm{CC} 8$ & & 1.559878 & \\
\hline $\operatorname{ccc} 8$ & & 102.634 & \\
\hline
\end{tabular}




$\begin{array}{lc}\text { dih8 } & 22.217 \\ \text { cc9 } & 1.535657 \\ \text { ccc9 } & 112.101 \\ \text { dih9 } & -126.221 \\ \text { cc10 } & 1.539962 \\ \text { ccc10 } & 110.943 \\ \text { dih10 } & 111.397 \\ \text { cc11 } & 1.482913 \\ \text { ccc11 } & 114.679 \\ \text { dih11 } & -152.201 \\ \text { hc12 } & 1.092006 \\ \text { hcc12 } & 113.024 \\ \text { dih12 } & -39.128 \\ \text { hc13 } & 1.094527 \\ \text { hcc13 } & 110.295 \\ \text { dih13 } & -159.952 \\ \text { hc14 } & 1.096167 \\ \text { hcc14 } & 109.226 \\ \text { dih14 } & 108.378 \\ \text { hc15 } & 1.093187 \\ \text { hcc15 } & 110.610 \\ \text { dih15 } & -131.533 \\ \text { hc16 } & 1.093944 \\ \text { hcc16 } & 110.813 \\ \text { dih16 } & 110.395 \\ \text { hc17 } & 1.095259 \\ \text { hcc17 } & 111.120 \\ \text { dih17 } & 175.530 \\ \text { hc18 } & 1.094433 \\ \text { hcc18 } & 111.235 \\ \text { dih18 } & -64.691 \\ \text { hc19 } & 1.094202 \\ \text { hcc19 } & 111.121 \\ \text { dih19 } & 55.521 \\ \text { hc20 } & 1.094783 \\ \text { hcc20 } & 110.960 \\ \text { dih20 } & -178.058 \\ \text { hc21 } & 1.093331 \\ \text { hcc21 } & 111.743 \\ \text { dih21 } & -57.831 \\ \text { hc22 } & 1.094826 \\ \text { hcc22 } & 110.952 \\ \text { dih22 } & 62.231 \\ \text { hc23 } & 1.098149 \\ \text { hcn23 } & 109.056 \\ \text { dih23 } & 88.896 \\ \text { hc24 } & 1.093341 \\ \text { hcn24 } & 110.746 \\ \text { dih24 } & -153.884 \\ \text { hc25 } & 1.094435 \\ \text { hcn25 } & 111.727 \\ \text { dih25 } & 130.259 \\ \text { hc26 } & 1.097965 \\ \text { hcn26 } & 110.210 \\ \text { dih26 } & -110.893 \\ \text { hc27 } & 1.095293 \\ \text { hcc27 } & 108.696 \\ & \\ & \end{array}$




$\begin{array}{lc}\text { dih2 } & 85.247 \\ \text { hc28 } & 1.085001 \\ \text { hcc28 } & 120.007 \\ \text { dih28 } & 50.013 \\ \text { hc29 } & 1.082235 \\ \text { hcc29 } & 121.998 \\ \text { dih29 } & -133.944\end{array}$

\section{$4, d$}

$\mathrm{C}$

C $\quad 1 \quad \mathrm{cc} 2$

c $\quad 2$ Cc $3 \quad 1 \quad c c 3$

n 3 nc4 2 ncc4

c 4 cn5 3 enc5

C 4 cn $6 \quad 3$ cnc 6

$\begin{array}{llll}\text { C } & 6 \mathrm{CC} 7 & 4 \mathrm{ccn} 7\end{array}$

$\begin{array}{llll}\text { C } & 7 \mathrm{CC} 8 & 6 \mathrm{cCC} 8\end{array}$

$\begin{array}{lll}\text { C } \quad 7 \mathrm{CC} 9 & 8 \operatorname{ccc} 9\end{array}$

C $1 \mathrm{cc} 10 \quad 2 \operatorname{ccc} 10$

C $1 \operatorname{ccll} 2 \operatorname{ccc} 11$

h 9 hcl2 7 hccl2

h 9 hc13

h 6 hc14

7 hec13

7 hec14

7 h⿻c15

8 hec16

h 7 hc16

h 8 hc17

h 8 hc18

h 3 hc19

h 2 hc20

h 2 hc21

h 10 hc22

h 10 hc23

h 10 hc24

h 11 hc25

h 11 hc26

h 11 hc27

h 5 hc28

h 5 hc 29

$7 \mathrm{hcc} 17$

7 hec18

8 hec19

3 hcc20

3 hec 21

1 hcc22

1 hcc23

1 hcc24

1 hcc25

1 hec 26

1 hcc27

4 hen 28

4 hen 29

1 dih 4

2 dih5

2 dih 6

3 dih 7

4 dih8

3 dih 9

3 dih 10

3 dih11

8 dih12

8 dih13

8 dih14

8 dih15

$3 \operatorname{dih} 16$

6 dih17

6 dih18

7 dih19

$8 \operatorname{dih} 20$

8 dih 21

$2 \operatorname{din} 22$

$2 \operatorname{dih} 23$

2 dih24

$2 \operatorname{din} 25$

$2 \operatorname{din} 26$

2 dih27

$3 \operatorname{din} 28$

$3 \operatorname{din} 29$

$\begin{array}{ll}\text { cc2 } & 1.576980 \\ \text { cc3 } & 1.529281 \\ \text { ccc3 } & 103.922 \\ \text { nc4 } & 1.455122 \\ \text { ncc4 } & 102.018 \\ \text { dih4 } & 31.587 \\ \text { cn5 } & 1.447240 \\ \text { cnc5 } & 105.701 \\ \text { dih5 } & -48.095 \\ \text { cn6 } & 1.447658 \\ \text { cnc6 } & 106.481 \\ \text { dih6 } & 178.346 \\ \text { cc7 } & 1.571406 \\ \text { ccn7 } & 103.324 \\ \text { dih7 } & -38.728 \\ \text { cc8 } & 1.585435 \\ \text { ccc8 } & 103.786 \\ \text { dih8 } & 13.209\end{array}$




$\begin{array}{lc}\text { cc9 } & 1.486152 \\ \text { ccc9 } & 113.105 \\ \text { dih9 } & 138.491 \\ \text { cc10 } & 1.534238 \\ \text { ccc10 } & 111.856 \\ \text { dih10 } & 113.452 \\ \text { cc11 } & 1.536502 \\ \text { ccc11 } & 110.179 \\ \text { dih11 } & -123.732 \\ \text { hc12 } & 1.084804 \\ \text { hcc12 } & 119.789 \\ \text { dih12 } & -75.181 \\ \text { hc13 } & 1.083191 \\ \text { hcc13 } & 121.513 \\ \text { dih13 } & 94.783 \\ \text { hc14 } & 1.093026 \\ \text { hcc14 } & 112.270 \\ \text { dih14 } & 133.810 \\ \text { hc15 } & 1.106638 \\ \text { hcc15 } & 109.560 \\ \text { dih15 } & -106.472 \\ \text { hc16 } & 1.093636 \\ \text { hcc16 } & 108.180 \\ \text { dih16 } & -100.685 \\ \text { hc17 } & 1.091713 \\ \text { hcc17 } & 111.588 \\ \text { dih17 } & 137.341 \\ \text { hc18 } & 1.092787 \\ \text { hcc18 } & 109.951 \\ \text { dih18 } & -102.214 \\ \text { hc19 } & 1.112318 \\ \text { hcc19 } & 108.571 \\ \text { dih19 } & 79.031 \\ \text { hc20 } & 1.092556 \\ \text { hcc20 } & 112.779 \\ \text { dih20 } & -93.660 \\ \text { hc21 } & 1.093414 \\ \text { hcc21 } & 110.101 \\ \text { dih21 } & 26.854 \\ \text { hc22 } & 1.094095 \\ \text { hcc22 } & 111.344 \\ \text { dih22 } & -54.426 \\ \text { hc23 } & 1.094488 \\ \text { hcc23 } & 110.955 \\ \text { dih23 } & 65.792 \\ \text { hc24 } & 1.095155 \\ \text { hcc24 } & 111.122 \\ \text { dih24 } & -174.488 \\ \text { hc25 } & 1.094690 \\ \text { hcc25 } & 111.041 \\ \text { dih25 } & -59.244 \\ \text { hc26 } & 1.094908 \\ \text { hcc26 } & 111.219 \\ \text { dih26 } & -179.275 \\ \text { hc27 } & 1.093238 \\ \text { hcc27 } & 110.284 \\ \text { dih27 } & 60.560 \\ & \end{array}$




$\begin{array}{ll}\text { hc28 } & 1.093786 \\ \text { hcn28 } & 111.927 \\ \text { dih28 } & 164.489 \\ \text { hc29 } & 1.107701 \\ \text { hcn29 } & 111.739 \\ \text { dih29 } & -74.499\end{array}$

$4, d$

C

n 1 nc2

c $2 \mathrm{cn} 3$

c $3 \mathrm{Cc} 4$

C $4 \mathrm{CC} 5$

c $3 \mathrm{cc} 6$

C $\quad 6 \mathrm{CC} 7$

c $7 \mathrm{CC} 8$

C $7 \mathrm{CC} 9$

C $\quad 7 \mathrm{CC} 10$

C $5 \mathrm{cc} 11$

h 4 hcl2

h 11 hc13

h 11 hc14

h 5 hc15

h 4 hcl 6

h 3 hcl 7

h 1 hcl 8

h 1 hcl9

h 6 hc20

h 6 hc21

h 8 hc22

h 8 hc23

h 9 hc24

h 9 hc25

h 9 hc26

h 10 hc27

h 10 hc28

h 10 hc2 9

$1 \mathrm{cnc} 3$

$2 \operatorname{ccn} 4$

$3 \operatorname{ccc} 5$

$2 \operatorname{ccn} 6$

$3 \operatorname{ccC} 7$

$6 \operatorname{ccc} 8$

$8 \operatorname{ccc} 9$

$8 \operatorname{ccc} 10$

$4 \operatorname{ccc} 11$

3 hccl2

5 hec13

5 hec14

4 hec15

$3 \mathrm{hcc} 16$

$2 \operatorname{hcn} 17$

2 hen18

2 hen 19

3 hec 20

3 hcc 21

2 hen 22

2 hen 23

7 hec 24

7 hec 25

7 hec 26

7 hcc2 7

7 hec 28

7 hec2 9

1 dih 4

2 dih5

1 dih 6

2 dih 7

3 dih8

2 dih9

2 dih10

3 dih11

$2 \operatorname{dih} 12$

4 dih13

4 dih14

3 dih15

2 dih16

8 dih17

8 dih18

8 dih19

$2 \operatorname{dih} 20$

$2 \operatorname{dih} 21$

3 dih22

3 dih23

8 dih24

8 dih25

8 dih 26

8 dih27

8 dih 28

8 dih29

$\begin{array}{lc}\text { nc2 } & 1.447313 \\ \text { cn3 } & 1.456391 \\ \text { cnc3 } & 105.593 \\ \text { cc4 } & 1.527584 \\ \text { ccn4 } & 102.506 \\ \text { dih4 } & 48.642 \\ \text { cc5 } & 1.595586 \\ \text { ccc5 } & 102.967 \\ \text { dih5 } & -31.842 \\ \text { cc6 } & 1.528412 \\ \text { ccn6 } & 102.022 \\ \text { dih6 } & 178.515 \\ \text { cc7 } & 1.576658 \\ \text { ccc7 } & 103.703 \\ \text { dih7 } & 32.410 \\ \text { cc8 } & 1.567014 \\ \text { ccc8 } & 103.404 \\ \text { dih8 } & -6.761\end{array}$




$\begin{array}{lc}\text { cc9 } & 1.534445 \\ \text { ccc9 } & 111.067 \\ \text { dih9 } & -141.403 \\ \text { cc10 } & 1.536298 \\ \text { ccc10 } & 110.077 \\ \text { dih10 } & 96.383 \\ \text { cc11 } & 1.484812 \\ \text { ccc11 } & 114.413 \\ \text { dih11 } & -118.146 \\ \text { hc12 } & 1.091123 \\ \text { hcc12 } & 113.094 \\ \text { dih12 } & -152.680 \\ \text { hc13 } & 1.084690 \\ \text { hcc13 } & 120.657 \\ \text { dih13 } & 81.360 \\ \text { hc14 } & 1.083387 \\ \text { hcc14 } & 121.028 \\ \text { dih14 } & -89.085 \\ \text { hc15 } & 1.093390 \\ \text { hcc15 } & 106.990 \\ \text { dih15 } & 121.051 \\ \text { hc16 } & 1.092808 \\ \text { hcc16 } & 110.837 \\ \text { dih16 } & 85.356 \\ \text { hc17 } & 1.112009 \\ \text { hcn17 } & 109.946 \\ \text { dih17 } & 66.369 \\ \text { hc18 } & 1.107625 \\ \text { hcn18 } & 111.944 \\ \text { dih18 } & -47.389 \\ \text { hc19 } & 1.092779 \\ \text { hcn19 } & 112.146 \\ \text { dih19 } & 74.094 \\ \text { hc20 } & 1.093465 \\ \text { hcc20 } & 110.146 \\ \text { dih20 } & -85.612 \\ \text { hc21 } & 1.092579 \\ \text { hcc21 } & 112.897 \\ \text { dih21 } & 153.719 \\ \text { hc22 } & 1.107574 \\ \text { hcn22 } & 111.769 \\ \text { dih22 } & -74.166 \\ \text { hc23 } & 1.093696 \\ \text { hcn23 } & 111.941 \\ \text { dih23 } & 164.703 \\ \text { hc24 } & 1.094079 \\ \text { hcc24 } & 111.303 \\ \text { dih24 } & 60.692 \\ \text { hc25 } & 1.094562 \\ \text { hcc25 } & 110.993 \\ \text { dih25 } & -179.103 \\ \text { hc26 } & 1.095113 \\ \text { hcc26 } & 111.116 \\ \text { dih26 } & -59.365 \\ \text { hc27 } & 1.094923 \\ \text { hcc27 } & 111.202 \\ \text { dih27 } & 67.464 \\ & \\ & \end{array}$




$\begin{array}{lc}\text { hc28 } & 1.094738 \\ \text { hcc28 } & 111.042 \\ \text { dih28 } & -172.525 \\ \text { hc29 } & 1.093259 \\ \text { hcc29 } & 110.318 \\ \text { dih29 } & -52.698\end{array}$

5

$\mathrm{C}$

C $\quad 1 \quad \mathrm{cc} 2$

n 2 nc3 1 ncc3

c $3 \mathrm{cn} 4 \quad 2 \mathrm{cnc} 4$

C $4 \operatorname{cc} 5 \quad 3 \operatorname{ccn} 5$

c $5 \operatorname{cc} 6 \quad 4 \operatorname{ccc} 6$

$\begin{array}{llll}\text { C } & 2 \mathrm{CC} 7 & 3 \mathrm{CCn} 7\end{array}$

$\begin{array}{lll}\text { C } & 5 \mathrm{CC} 8 & 4 \mathrm{CCC} 8\end{array}$

$\begin{array}{lll}\text { c } & 5 \operatorname{cc} 9 & 4 \operatorname{ccc} 9\end{array}$

c $9 \operatorname{ccl} 10 \quad 5 \operatorname{ccc} 10$

C $10 \mathrm{ccll} \quad 9 \mathrm{ccc} 11$

C $11 \quad \mathrm{cc} 12 \quad 10 \quad \mathrm{ccc} 12$

h 9 hc13

h 9 hcl 4

h 6 hc15

h 6 hc1 6

h 6 hc17

h 8 hc18

h 8 hc19

h 8 hc20

h 4 hc21

h 4 hc22

h 7 hc23

h 7 hc24

h 2 hc25

h 1 hc26

h 1 hc27

h 10 hc28

h 10 hc2 9

h 11 hc30

h 12 hc31

h 12 hc32

5 hec13

5 hec14

5 hec15

5 hec16

$5 \operatorname{hcc} 17$

5 hec18

5 hcc19

5 hcc 20

3 hen 21

3 hen 22

2 hcc23

2 hcc2 4

7 hcc 25

2 hcc 26

2 hcc27

9 hcc28

9 hec 29

10 hcc 30

11 hcc31

11 hcc32

1 dih 4

$2 \operatorname{din} 5$

3 dih 6

4 dih7

3 dih 8

3 dih 9

4 dih10

$5 \operatorname{dih} 11$

9 dih12

4 dih13

4 dih14

4 dih15

4 dih16

4 dih17

4 dih18

4 dih19

4 dih20

2 dih21

$2 \operatorname{dih} 22$

$1 \operatorname{dih} 23$

1 dih24

3 dih25

7 dih 26

7 dih27

5 dih28

5 dih29

9 dih 30

10 dih31

$10 \operatorname{dih} 32$

$\begin{array}{lc}\text { cc2 } & 1.452705 \\ \mathrm{nc} 3 & 1.478168 \\ \mathrm{ncc} 3 & 118.675 \\ \mathrm{cn} 4 & 1.463133 \\ \text { cnc4 } & 117.423 \\ \text { dih4 } & 140.018 \\ \text { cc5 } & 1.547801 \\ \text { ccn5 } & 113.666 \\ \text { dih5 } & -100.416 \\ \text { cc6 } & 1.539822 \\ \text { ccc6 } & 109.994 \\ \text { dih6 } & 60.395 \\ \text { cc7 } & 1.519465 \\ \text { ccn7 } & 57.695 \\ \text { dih7 } & -106.880 \\ & \end{array}$




\begin{tabular}{lc} 
cc8 & 1.540577 \\
ccc8 & 107.033 \\
dih8 & 179.140 \\
cc9 & 1.550813 \\
ccc9 & 108.082 \\
dih9 & -61.400 \\
cc10 & 1.540768 \\
ccc10 & 116.678 \\
dih10 & 176.540 \\
cc11 & 1.503650 \\
ccc11 & 111.931 \\
dih11 & 175.075 \\
cc12 & 1.329761 \\
ccc12 & 125.478 \\
dih12 & 118.643 \\
hc13 & 1.098151 \\
hcc13 & 107.916 \\
dih13 & -60.978 \\
hc14 & 1.092930 \\
hcc14 & 108.161 \\
dih14 & 53.431 \\
hc15 & 1.095054 \\
hcc15 & 110.718 \\
dih15 & 54.910 \\
hc16 & 1.092886 \\
hcc16 & 111.193 \\
dih16 & 173.981 \\
hc17 & 1.092484 \\
hcc17 & 111.522 \\
dih17 & -65.297 \\
hc18 & 1.094708 \\
hcc18 & 110.941 \\
dih18 & -59.540 \\
hc19 & 1.094826 \\
hcc19 & 111.292 \\
dih19 & 60.514 \\
hc20 & 1.092539 \\
hcc20 & 111.529 \\
dih20 & -179.062 \\
hc21 & 1.104565 \\
hcn21 & 111.375 \\
dih21 & 23.299 \\
hc22 & 1.096643 \\
hcn22 & 106.984 \\
dih22 & 139.773 \\
hc23 & 1.085188 \\
hcc23 & 119.419 \\
dih23 & 0.376 \\
hc24 & 1.089769 \\
hcc24 & 116.422 \\
dih24 & -145.593 \\
hc25 & 1.089552 \\
hcc25 & 113.202 \\
dih25 & -106.603 \\
hc26 & 1.082024 \\
hcc26 & 120.645 \\
dih26 & 129.575 \\
ih & \\
\hline &
\end{tabular}




$\begin{array}{ll}\text { hc27 } & 1.082285 \\ \text { hcc27 } & 119.994 \\ \text { dih27 } & -44.840 \\ \text { hc28 } & 1.093881 \\ \text { hcc28 } & 110.403 \\ \text { dih28 } & -63.086 \\ \text { hc29 } & 1.097417 \\ \text { hcc29 } & 109.743 \\ \text { dih29 } & 54.420 \\ \text { hc30 } & 1.089793 \\ \text { hcc30 } & 115.698 \\ \text { dih30 } & -60.661 \\ \text { hc31 } & 1.084376 \\ \text { hcc31 } & 121.726 \\ \text { dih31 } & -179.601 \\ \text { hc32 } & 1.086206 \\ \text { hcc32 } & 121.552 \\ \text { dih32 } & 0.452 \\ & \end{array}$

6

C

c $\quad 1 \quad \mathrm{cc} 2$

c $2 \mathrm{cc} 3$

n 3 nc4

c $4 \mathrm{cn} 5$

c $5 \mathrm{cc} 6$

C $\quad 6 \mathrm{cc} 7$

c $6 \mathrm{cc} 8$

c $8 \mathrm{cc} 9$

C $9 \mathrm{cc} 10$

C $10 \mathrm{cc} 11$

C $6 \mathrm{cc} 12$

h 8 hc13

h 8 hcl 4

h 12 hc15

h 12 hcl6

h 12 hcl7

h 7 hcl 8

h 7 hc19

h 7 hc20

h 5 hc21

h 5 hc22

h 3 hc23

h 3 hc24

h 2 hc25

h 1 hc26

h 1 hc27

h 9 hc28

h 9 hc2 9

h 10 hc30

h 11 hc31

h 11 hc32

$\begin{array}{ll}\mathrm{cc} 2 & 1.328049 \\ \mathrm{cc} 3 & 1.503943 \\ \mathrm{ccc} 3 & 124.632\end{array}$




$\begin{array}{lc}\text { nc4 } & 1.443287 \\ \text { ncc4 } & 111.835 \\ \text { dih4 } & 133.535 \\ \text { cn5 } & 1.437735 \\ \text { cnc5 } & 113.081 \\ \text { dih5 } & -167.172 \\ \text { cc6 } & 1.547067 \\ \text { ccn6 } & 113.374 \\ \text { dih6 } & -175.863 \\ \text { cc7 } & 1.539141 \\ \text { ccc7 } & 107.697 \\ \text { dih7 } & 178.447 \\ \text { cc8 } & 1.550269 \\ \text { ccc8 } & 107.843 \\ \text { dih8 } & -61.574 \\ \text { cc9 } & 1.540735 \\ \text { ccc9 } & 116.689 \\ \text { dih9 } & 177.034 \\ \text { cc10 } & 1.503708 \\ \text { ccc10 } & 111.970 \\ \text { dih10 } & 175.693 \\ \text { cc11 } & 1.329702 \\ \text { ccc11 } & 125.473 \\ \text { dih11 } & 119.140 \\ \text { cc12 } & 1.539017 \\ \text { ccc12 } & 108.785 \\ \text { dih12 } & 59.454 \\ \text { hc13 } & 1.098532 \\ \text { hcc13 } & 108.011 \\ \text { dih13 } & -60.462 \\ \text { hc14 } & 1.093813 \\ \text { hcc14 } & 108.130 \\ \text { dih14 } & 54.068 \\ \text { hc15 } & 1.095236 \\ \text { hcc15 } & 110.559 \\ \text { dih15 } & 58.008 \\ \text { hc16 } & 1.092599 \\ \text { hcc16 } & 111.545 \\ \text { dih16 } & 177.400 \\ \text { hc17 } & 1.092053 \\ \text { hcc17 } & 110.732 \\ \text { dih17 } & -61.541 \\ \text { hc18 } & 1.094840 \\ \text { hcc18 } & 110.904 \\ \text { dih18 } & -58.123 \\ \text { hc19 } & 1.095050 \\ \text { hcc19 } & 111.349 \\ \text { dih19 } & 61.927 \\ \text { hc20 } & 1.092542 \\ \text { hcc20 } & 111.587 \\ \text { dih20 } & -177.589 \\ \text { hc21 } & 1.105773 \\ \text { hcn21 } & 109.813 \\ \text { dih21 } & -53.218 \\ \text { hc22 } & 1.105449 \\ \text { hcn22 } & 109.329 \\ \text { dih22 } & 61.759 \\ & \\ & \end{array}$




$\begin{array}{lc}\text { hc23 } & 1.102603 \\ \text { hcc23 } & 110.577 \\ \text { dih23 } & 7.708 \\ \text { hc24 } & 1.107457 \\ \text { hcc24 } & 109.180 \\ \text { dih24 } & -107.881 \\ \text { hc25 } & 1.087057 \\ \text { hcc25 } & 114.985 \\ \text { dih25 } & -46.433 \\ \text { hc26 } & 1.083991 \\ \text { hcc26 } & 121.647 \\ \text { dih26 } & 179.065 \\ \text { hc27 } & 1.086263 \\ \text { hcc27 } & 121.697 \\ \text { dih27 } & -0.928 \\ \text { hc28 } & 1.093814 \\ \text { hcc28 } & 110.414 \\ \text { dih28 } & -62.377 \\ \text { hc29 } & 1.097378 \\ \text { hcc29 } & 109.643 \\ \text { dih29 } & 55.089 \\ \text { hc30 } & 1.089813 \\ \text { hcc30 } & 115.696 \\ \text { dih30 } & -60.228 \\ \text { hc31 } & 1.084360 \\ \text { hcc31 } & 121.716 \\ \text { dih31 } & -179.658 \\ \text { hc32 } & 1.086182 \\ \text { hcc32 } & 121.564 \\ \text { dih32 } & 0.373 \\ \text { r } & \end{array}$

\section{7}

C

c $\quad 1 \quad \mathrm{cc} 2$

n 2 nc3

c $3 \mathrm{cn} 4$

c $4 \mathrm{cc} 5$

c $5 \mathrm{cc} 6$

c $2 \mathrm{CC} 7$

c $3 \mathrm{cn} 8$

c $8 \mathrm{cc} 9$

c $9 \mathrm{cc} 10$

C $5 \mathrm{cc} 11$

c $\quad 5 \mathrm{cc} 12$

h 6 hc13

h 6 hc14

h 12 hc15

h 12 hc16

h 12 hc17

h 11 hcl8

h 11 hc19

h 11 hc20

h 4 hc21

h 4 hc22

h 8 hc23

h 8 hc24

h 9 hc25

\begin{tabular}{|c|c|}
\hline 1 ncc3 & \\
\hline $2 \operatorname{cnc} 4$ & 1 dih4 \\
\hline $3 \operatorname{ccn} 5$ & 2 dih5 \\
\hline $4 \operatorname{ccc} 6$ & 3 dih 6 \\
\hline $3 \operatorname{ccn} 7$ & 4 dih7 \\
\hline $2 \operatorname{cnc} 8$ & 1 dih8 \\
\hline $3 \operatorname{ccn} 9$ & 2 dih9 \\
\hline $8 \operatorname{ccc} 10$ & 3 dih10 \\
\hline $4 \operatorname{ccc} 11$ & 3 dihl1 \\
\hline $4 \operatorname{ccc} 12$ & 3 dih12 \\
\hline 5 hсc13 & 4 dih13 \\
\hline 5 hсc14 & 4 dih14 \\
\hline 5 hсc15 & 4 dih15 \\
\hline 5 hсc16 & $4 \operatorname{dih} 16$ \\
\hline 5 hсc17 & 4 dih17 \\
\hline 5 hсc18 & $4 \operatorname{dih} 18$ \\
\hline 5 hсc19 & 4 dih19 \\
\hline 5 hсc 20 & 4 dih20 \\
\hline $3 h \mathrm{~h} n 21$ & $2 \operatorname{dih} 21$ \\
\hline 3 hen 22 & $2 \operatorname{dih} 22$ \\
\hline $3 h \mathrm{hen} 23$ & $2 \operatorname{dih} 23$ \\
\hline $3 h \mathrm{hcn} 24$ & $2 \operatorname{din} 24$ \\
\hline 8 hсc25 & 3 dih25 \\
\hline
\end{tabular}




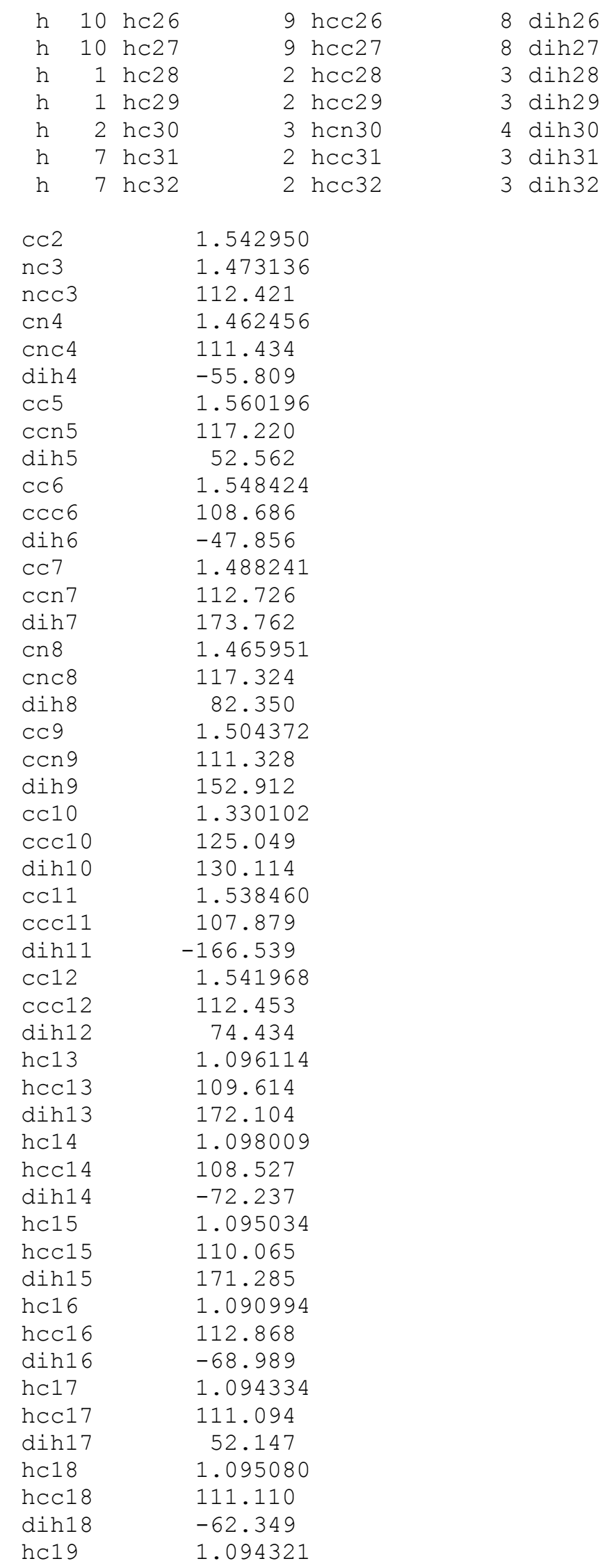




$\begin{array}{ll}\text { hcc19 } & 111.387 \\ \text { dih19 } & 57.873 \\ \text { hc20 } & 1.094550 \\ \text { hcc20 } & 110.922 \\ \text { dih20 } & 177.977 \\ \text { hc21 } & 1.093690 \\ \text { hcn21 } & 108.519 \\ \text { dih21 } & 177.528 \\ \text { hc22 } & 1.098545 \\ \text { hcn22 } & 107.054 \\ \text { dih22 } & -67.815 \\ \text { hc23 } & 1.099692 \\ \text { hcn23 } & 114.671 \\ \text { dih23 } & -82.479 \\ \text { hc24 } & 1.095984 \\ \text { hcn24 } & 106.597 \\ \text { dih24 } & 34.825 \\ \text { hc25 } & 1.088091 \\ \text { hcc25 } & 114.893 \\ \text { dih25 } & -50.243 \\ \text { hc26 } & 1.084290 \\ \text { hcc26 } & 121.731 \\ \text { dih26 } & 178.204 \\ \text { hc27 } & 1.086279 \\ \text { hcc27 } & 121.565 \\ \text { dih27 } & -1.301 \\ \text { hc28 } & 1.095485 \\ \text { hcc28 } & 109.539 \\ \text { dih28 } & -65.578 \\ \text { hc29 } & 1.095664 \\ \text { hcc29 } & 109.659 \\ \text { dih29 } & 177.703 \\ \text { hc30 } & 1.108624 \\ \text { hcn30 } & 104.280 \\ \text { dih30 } & 58.321 \\ \text { hc31 } & 1.083516 \\ \text { hcc31 } & 119.715 \\ \text { dih31 } & -42.097 \\ \text { hc32 } & 1.082842 \\ \text { hcc32 } & 120.732 \\ \text { dih32 } & 149.800 \\ & \end{array}$

8

C

C $\quad 1 \quad \mathrm{Cc} 2$

n 2 nc3

c $3 \mathrm{cn} 4$

C $4 \mathrm{CC} 5$

c $3 \mathrm{cn} 6$

C $6 \mathrm{cc} 7$

C $\quad 7 \mathrm{cc} 8$

C $8 \mathrm{cc} 9$

C $\quad 7 \mathrm{cc} 10$

C $\quad 7 \mathrm{cc} 11$

$\begin{array}{ll}1 \operatorname{ncc} 3 & 1 \text { dih4 } \\ 2 \operatorname{cnc} 4 & 2 \operatorname{dih} 5 \\ 3 \operatorname{ccn} 5 & 1 \text { dih6 } \\ 2 \operatorname{cnc} 6 & 2 \operatorname{dih} 7 \\ 3 \operatorname{ccn} 7 & 3 \operatorname{dih} 8 \\ 6 \operatorname{ccc} 8 & 6 \operatorname{dih} 9 \\ 7 \operatorname{ccc} 9 & 3 \operatorname{dih} 10 \\ 6 \operatorname{ccc} 10 & 3 \operatorname{dih} 11\end{array}$




\begin{tabular}{|c|c|c|c|c|}
\hline C & $5 \operatorname{ccl} 2$ & $4 \operatorname{ccc} 12$ & 3 & $\operatorname{dih} 12$ \\
\hline $\mathrm{h}$ & 8 hc13 & $9 \mathrm{hcc} 13$ & 2 & $\operatorname{dih} 13$ \\
\hline $\mathrm{h}$ & 8 hcl 4 & $9 \operatorname{hccl} 4$ & 2 & $\operatorname{dih} 14$ \\
\hline $\mathrm{h}$ & 1 hc15 & 7 hcc15 & 6 & dih15 \\
\hline $\mathrm{h}$ & 1 hcl 6 & 7 hcc1 6 & 6 & $\operatorname{dih} 16$ \\
\hline $\mathrm{h}$ & 1 hc17 & $7 \mathrm{hcc} 17$ & 6 & $\operatorname{dih} 17$ \\
\hline $\mathrm{h}$ & 0 hc18 & 7 hсc18 & 6 & $\operatorname{dih} 18$ \\
\hline $\mathrm{h}$ & 0 hc19 & 7 hccl9 & 6 & $\operatorname{dih} 19$ \\
\hline $\mathrm{h}$ & 0 hc20 & 7 hсc20 & 6 & $\operatorname{dih} 20$ \\
\hline $\mathrm{h}$ & 6 hc21 & $3 \operatorname{hcn} 21$ & 2 & $\operatorname{dih} 21$ \\
\hline $\mathrm{h}$ & 6 hс22 & 3 hсn22 & 2 & $\operatorname{dih} 22$ \\
\hline $\mathrm{h}$ & 4 hс23 & $3 \operatorname{hcn} 23$ & 2 & $\operatorname{dih} 23$ \\
\hline $\mathrm{h}$ & 4 hс24 & $3 \operatorname{hcn} 24$ & 2 & $\operatorname{dih} 24$ \\
\hline $\mathrm{h}$ & 5 hс2 5 & 4 hсc25 & 3 & $\operatorname{dih} 25$ \\
\hline $\mathrm{h}$ & 2 hc2 6 & 5 hсс2 6 & 4 & $\operatorname{dih} 26$ \\
\hline $\mathrm{h}$ & 2 hс27 & 5 hсc27 & 4 & $\operatorname{dih} 27$ \\
\hline $\mathrm{h}$ & 9 hc28 & 2 hсc 28 & 3 & $\operatorname{dih} 28$ \\
\hline $\mathrm{h}$ & 9 hc29 & $2 \operatorname{hcc} 29$ & 3 & $\operatorname{dih} 29$ \\
\hline $\mathrm{h}$ & 2 hс 30 & 9 hсc30 & 8 & $\operatorname{dih} 30$ \\
\hline $\mathrm{h}$ & 1 hс31 & $2 \operatorname{hcc} 31$ & 9 & $\operatorname{dih} 31$ \\
\hline $\mathrm{h}$ & 1 hс 32 & 2 hсc 32 & 9 & $\operatorname{dih} 32$ \\
\hline $\mathrm{Cc} 2$ & & 1.549933 & & \\
\hline nc3 & & 1.468907 & & \\
\hline ncc3 & & 103.954 & & \\
\hline $\mathrm{cn} 4$ & & 1.453086 & & \\
\hline Cnc4 & & 109.425 & & \\
\hline dih4 & & -25.382 & & \\
\hline CC5 5 & & 1.538387 & & \\
\hline $\operatorname{ccn} 5$ & & 101.757 & & \\
\hline dih5 & & 40.713 & & \\
\hline $\operatorname{cn} 6$ & & 1.451587 & & \\
\hline $\operatorname{cnc} 6$ & & 116.604 & & \\
\hline $\operatorname{dih} 6$ & & -169.432 & & \\
\hline $\mathrm{CC} 7$ & & 1.555248 & & \\
\hline $\operatorname{ccn} 7$ & & 116.679 & & \\
\hline $\operatorname{dih} 7$ & & 47.160 & & \\
\hline $\mathrm{cc} 8$ & & 1.548269 & & \\
\hline $\operatorname{ccc} 8$ & & 107.659 & & \\
\hline dih8 & & -48.172 & & \\
\hline $\operatorname{cc} 9$ & & 1.534631 & & \\
\hline $\operatorname{ccc} 9$ & & 112.951 & & \\
\hline dihg & & 52.625 & & \\
\hline cc10 & & 1.537089 & & \\
\hline $\operatorname{ccc} 10$ & & 108.580 & & \\
\hline dih10 & & -166.681 & & \\
\hline $\operatorname{cc} 11$ & & 1.540010 & & \\
\hline $\operatorname{ccc} 11$ & & 111.512 & & \\
\hline dih11 & & 73.802 & & \\
\hline $\mathrm{cc} 12$ & & 1.486220 & & \\
\hline $\operatorname{ccc} 12$ & & 115.503 & & \\
\hline $\operatorname{dih} 12$ & & -164.289 & & \\
\hline hc13 & & 1.096119 & & \\
\hline hecl 3 & & 110.387 & & \\
\hline dih13 & & -178.903 & & \\
\hline hc14 & & 1.098370 & & \\
\hline hecl 4 & & 108.897 & & \\
\hline
\end{tabular}




$\begin{array}{ll}\text { dih14 } & \text { 64.747 } \\ \text { hc15 } & 1.095066 \\ \text { hcc15 } & 110.590 \\ \text { dih15 } & 178.461 \\ \text { hc16 } & 1.090825 \\ \text { hcc16 } & 112.723 \\ \text { dih16 } & -61.069 \\ \text { hc17 } & 1.094694 \\ \text { hcc17 } & 110.703 \\ \text { dih17 } & 59.315 \\ \text { hc18 } & 1.095153 \\ \text { hcc18 } & 111.161 \\ \text { dih18 } & -61.583 \\ \text { hc19 } & 1.094377 \\ \text { hcc19 } & 111.354 \\ \text { dih19 } & 58.680 \\ \text { hc20 } & 1.094585 \\ \text { hcc20 } & 110.940 \\ \text { dih20 } & 178.713 \\ \text { hc21 } & 1.095116 \\ \text { hcn21 } & 108.563 \\ \text { dih21 } & 172.109 \\ \text { hc22 } & 1.100498 \\ \text { hcn22 } & 107.558 \\ \text { dih22 } & -73.092 \\ \text { hc23 } & 1.094863 \\ \text { hcn23 } & 110.545 \\ \text { dih23 } & 160.495 \\ \text { hc24 } & 1.105306 \\ \text { hcn24 } & 115.115 \\ \text { dih24 } & -77.564 \\ \text { hc25 } & 1.102799 \\ \text { hcc25 } & 106.323 \\ \text { dih25 } & 74.067 \\ \text { hc26 } & 1.083959 \\ \text { hcc26 } & 120.629 \\ \text { dih26 } & 161.244 \\ \text { hc27 } & 1.084071 \\ \text { hcc27 } & 120.952 \\ \text { dih27 } & -29.773 \\ \text { hc28 } & 1.095534 \\ \text { hcc28 } & 109.367 \\ \text { dih28 } & -71.560 \\ \text { hc29 } & 1.096137 \\ \text { hcc29 } & 109.494 \\ \text { dih29 } & 172.186 \\ \text { hc30 } & 1.099852 \\ \text { hcc30 } & 107.721 \\ \text { dih30 } & -68.910 \\ \text { hc31 } & 1.091877 \\ \text { hcc31 } & 112.332 \\ \text { dih31 } & 115.218 \\ \text { hc32 } & 1.093947 \\ \text { hcc32 } & 110.171 \\ \text { dih32 } & -4.383 \\ & \end{array}$


C

C $1 \mathrm{cc} 2$

C $2 \mathrm{cc} 3$

n 3 nc 4

C $4 \mathrm{cn} 5$

C $5 \mathrm{cc} 6$

C $\quad 6 \mathrm{CC} 7$

C $7 \mathrm{cc} 8$

C $8 \mathrm{cc} 9$

C $9 \mathrm{cc} 10$

h $6 \mathrm{hcl} 1$

h 6 hel 2

h 7 hel 3

h 7 hel 4

h 5 hel 5

h 5 hcl 6

h 3 hel 7

h 3 hel 8

h 2 hel 9

h 1 hc20

h 1 hc2 1

h 8 hc22

h 8 hc23

h 9 hc24

h 10 hc25

h 10 hc26

$1 \mathrm{CCC} 3$

2 ncc 4

3 cnc5

$4 \operatorname{ccn} 6$

$5 \operatorname{ccc} 7$

$6 \operatorname{ccc} 8$

$7 \operatorname{ccc} 9$

$8 \operatorname{ccc} 10$

5 hccll

5 hccl2

6 hccl 3

6 hecl 4

4 hen 15

4 hcn 16

2 hccl 7

2 hccl 8

4 hen19

2 hcc20

2 hcc2 1

7 hсc22

7 hcc23

8 hcc 24

9 hcc2 5

9 hcc2 6

1 dih4

2 dih5

3 dih 6

4 dih 7

5 dih8

6 dih9

7 dih 10

4 dih11

4 dih12

5 dih13

5 dih 14

2 dih 15

2 dih16

1 dih 17

1 dih18

5 dih19

4 dih20

4 dih21

6 dih 22

6 dih23

7 dih24

8 dih25

$\begin{array}{lc}\text { cc2 } & 1.452536 \\ \text { cc3 } & 1.522486 \\ \text { ccc3 } & 123.339 \\ \text { nc4 } & 1.445439 \\ \text { ncc4 } & 59.605 \\ \text { dih4 } & 105.317 \\ \text { cn5 } & 1.463236 \\ \text { cnc5 } & 116.567 \\ \text { dih5 } & 107.333 \\ \text { cc6 } & 1.529866 \\ \text { ccn6 } & 111.531 \\ \text { dih6 } & -160.622 \\ \text { cc7 } & 1.532512 \\ \text { ccc7 } & 113.715 \\ \text { dih7 } & -62.972 \\ \text { cc8 } & 1.538879 \\ \text { ccc8 } & 112.937 \\ \text { dih8 } & 178.134 \\ \text { cc9 } & 1.502733 \\ \text { ccc9 } & 113.008 \\ \text { dih9 } & 177.936 \\ \text { cc10 } & 1.329703 \\ \text { ccc10 } & 125.397 \\ \text { dih10 } & 119.137 \\ \text { hc11 } & 1.096851 \\ \text { hcc11 } & 108.889 \\ \text { dih11 } & 59.578 \\ \text { hc12 } & 1.096917\end{array}$




$\begin{array}{lc}\text { hcc12 } & 108.282 \\ \text { dih12 } & 174.803 \\ \text { hc13 } & 1.097664 \\ \text { hcc13 } & 109.141 \\ \text { dih13 } & -60.082 \\ \text { hc14 } & 1.092869 \\ \text { hcc14 } & 109.237 \\ \text { dih14 } & 55.824 \\ \text { hc15 } & 1.104377 \\ \text { hcn15 } & 111.415 \\ \text { dih15 } & 33.464 \\ \text { hc16 } & 1.096628 \\ \text { hcn16 } & 107.280 \\ \text { dih16 } & 150.167 \\ \text { hc17 } & 1.085090 \\ \text { hcc17 } & 119.407 \\ \text { dih17 } & 0.322 \\ \text { hc18 } & 1.089801 \\ \text { hcc18 } & 116.373 \\ \text { dih18 } & -145.614 \\ \text { hc19 } & 1.090260 \\ \text { hcn19 } & 115.707 \\ \text { dih19 } & -5.910 \\ \text { hc20 } & 1.082042 \\ \text { hcc20 } & 120.651 \\ \text { dih20 } & -162.806 \\ \text { hc21 } & 1.082163 \\ \text { hcc21 } & 119.970 \\ \text { dih21 } & 22.402 \\ \text { hc22 } & 1.095737 \\ \text { hcc22 } & 109.700 \\ \text { dih22 } & -59.510 \\ \text { hc23 } & 1.099081 \\ \text { hcc23 } & 108.739 \\ \text { dih23 } & 56.688 \\ \text { hc24 } & 1.089730 \\ \text { hcc24 } & 115.733 \\ \text { dih24 } & -60.149 \\ \text { hc25 } & 1.084345 \\ \text { hcc25 } & 121.766 \\ \text { dih25 } & -179.549 \\ \text { hc26 } & 1.086240 \\ \text { hcc26 } & 121.502 \\ \text { dih26 } & 0.520 \\ & \end{array}$

10

C

C $\quad 1 \quad \mathrm{Cc} 2$

c $\quad 2 \mathrm{cc} 3$

n $3 \mathrm{nc} 4$

c 4 cn 5

c $5 \mathrm{cc} 6$

C $\quad 6 \mathrm{CC} 7$

C $\quad 7 \mathrm{CC} 8$

c 8 CC 9

C $9 \mathrm{cc} 10$

h 6 hcll

$\begin{array}{ll}1 \operatorname{ccc} 3 & \\ 2 \operatorname{ncc} 4 & 1 \text { dih4 } \\ 3 \operatorname{cnc} 5 & 2 \text { dih5 } \\ 4 \operatorname{ccn} 6 & 3 \text { dih6 } \\ 5 \operatorname{ccc} 7 & 4 \text { dih7 } \\ 6 \operatorname{ccc} 8 & 5 \text { dih8 } \\ 7 \operatorname{ccc} 9 & 6 \text { dih9 } \\ 8 \operatorname{ccc} 10 & 7 \text { dih10 } \\ 5 \operatorname{hcc} 11 & 4 \text { dih11 }\end{array}$




\begin{tabular}{|c|c|c|}
\hline $\mathrm{h}$ & $5 \mathrm{hcc} 12$ & 4 dih12 \\
\hline $\mathrm{h}$ & $6 \mathrm{hcc} 13$ & 5 dih13 \\
\hline $\mathrm{h}$ & $6 \mathrm{hcc} 14$ & 5 dih14 \\
\hline $\mathrm{h}$ & $4 \mathrm{hcn} 15$ & 3 dih15 \\
\hline $\mathrm{h}$ & $4 h \operatorname{cn} 16$ & 3 dih16 \\
\hline $\mathrm{h}$ & $2 \operatorname{hcc} 17$ & $1 \operatorname{dih} 17$ \\
\hline $\mathrm{h}$ & $2 \mathrm{hcc} 18$ & 1 dih18 \\
\hline $\mathrm{h}$ & $3 \mathrm{hcc} 19$ & 4 dih19 \\
\hline $\mathrm{h}$ & 2 hсc20 & 3 dih20 \\
\hline $\mathrm{h}$ & $2 \operatorname{hcc} 21$ & 3 dih21 \\
\hline $\mathrm{h}$ & 7 hсс22 & 6 dih22 \\
\hline $\mathrm{h}$ & 7 hсс23 & 6 dih23 \\
\hline $\mathrm{h}$ & 8 hсc24 & 7 dih24 \\
\hline 10 & 9 hсc25 & 8 dih25 \\
\hline 10 & 9 hсc26 & 8 dih26 \\
\hline $\mathrm{cc} 2$ & 1.328043 & \\
\hline $\mathrm{cc} 3$ & 1.503967 & \\
\hline $\mathrm{ccc} 3$ & 124.655 & \\
\hline $\mathrm{n} C 4$ & 1.443599 & \\
\hline $\mathrm{ncC} 4$ & 111.720 & \\
\hline dih4 & 133.077 & \\
\hline cn 5 & 1.440932 & \\
\hline cnc5 & 113.073 & \\
\hline dih5 & -167.534 & \\
\hline $\operatorname{cc} 6$ & 1.530103 & \\
\hline $\operatorname{ccn} 6$ & 111.928 & \\
\hline dih 6 & -179.206 & \\
\hline $\mathrm{CC} 7$ & 1.531491 & \\
\hline $\mathrm{CCC} 7$ & 113.025 & \\
\hline $\operatorname{dih} 7$ & -64.443 & \\
\hline $\operatorname{cc} 8$ & 1.538653 & \\
\hline $\operatorname{ccc} 8$ & 113.112 & \\
\hline dih8 & 177.196 & \\
\hline $\operatorname{cc} 9$ & 1.502762 & \\
\hline $\operatorname{ccc} 9$ & 113.051 & \\
\hline dihg & 177.849 & \\
\hline cc10 & 1.329645 & \\
\hline $\operatorname{ccc} 10$ & 125.404 & \\
\hline dih10 & 118.939 & \\
\hline hc11 & 1.096806 & \\
\hline hecl1 & 108.879 & \\
\hline dih11 & 173.061 & \\
\hline hc12 & 1.095736 & \\
\hline hec12 & 108.321 & \\
\hline dih12 & 57.262 & \\
\hline hc13 & 1.098086 & \\
\hline hec13 & 109.274 & \\
\hline dih13 & -60.835 & \\
\hline hc14 & 1.093912 & \\
\hline hec1 4 & 108.931 & \\
\hline dih14 & 55.147 & \\
\hline hc15 & 1.104865 & \\
\hline hen15 & 109.424 & \\
\hline dih15 & -56.851 & \\
\hline hc16 & 1.105198 & \\
\hline hen1 6 & 109.802 & \\
\hline
\end{tabular}




$\begin{array}{lc}\text { dih16 } & 58.112 \\ \text { hc17 } & 1.102637 \\ \text { hcc17 } & 110.584 \\ \text { dih17 } & 7.330 \\ \text { hc18 } & 1.107599 \\ \text { hcc18 } & 109.183 \\ \text { dih18 } & -108.309 \\ \text { hc19 } & 1.087040 \\ \text { hcc19 } & 114.955 \\ \text { dih19 } & -46.737 \\ \text { hc20 } & 1.084008 \\ \text { hcc20 } & 121.645 \\ \text { dih20 } & 179.209 \\ \text { hc21 } & 1.086262 \\ \text { hcc21 } & 121.698 \\ \text { dih21 } & -0.810 \\ \text { hc22 } & 1.095570 \\ \text { hcc22 } & 109.641 \\ \text { dih22 } & -59.549 \\ \text { hc23 } & 1.099028 \\ \text { hcc23 } & 108.684 \\ \text { dih23 } & 56.597 \\ \text { hc24 } & 1.089761 \\ \text { hcc24 } & 115.744 \\ \text { dih24 } & -60.358 \\ \text { hc25 } & 1.084347 \\ \text { hcc25 } & 121.752 \\ \text { dih25 } & -179.545 \\ \text { hc26 } & 1.086170 \\ \text { hcc26 } & 121.521 \\ \text { dih26 } & 0.519 \\ & \end{array}$

11

C

C $\quad 1 \quad \mathrm{CC} 2$

c $2 \mathrm{cc} 3$

n 3 nc4

c 4 cn 5

C $5 \mathrm{cc} 6$

C $3 \mathrm{CC} 7$

C $4 \mathrm{cn} 8$

c $8 \mathrm{cc} 9$

c $9 \mathrm{cc} 10$

h 6 hcll

h 6 hcl2

h 1 hc13

h 1 hc14

h 5 hcl 5

h 5 hc16

h 8 hcl 7

h 8 hcl 8

h 9 hcl 9

h 10 hc20

h 10 hc21

h 2 hc22

h 2 hc23

$\begin{array}{ll}1 \text { ccc3 } & \\ 2 \text { ncc } 4 & \text { dih4 } \\ 3 \text { cnc5 } & 2 \text { dih5 } \\ 4 \text { ccn } 6 & 3 \text { dih6 } \\ 2 \text { ccc7 } & 1 \text { dih7 } \\ 3 \text { cnc8 } & 2 \text { dih8 } \\ 4 \text { ccn } 9 & 3 \text { dih9 } \\ 8 \text { ccc10 } & 4 \text { dih10 } \\ 5 \text { hcc11 } & 4 \text { dih11 } \\ 5 \text { hcc12 } & 4 \text { dih12 } \\ 2 \text { hcc13 } & 3 \text { dih13 } \\ 2 \text { hcc14 } & 3 \text { dih14 } \\ 4 \text { hcn15 } & 3 \text { dih15 } \\ 4 \text { hcn16 } & 3 \text { dih16 } \\ 4 \text { hcn17 } & 3 \text { dih17 } \\ 4 \text { hcn18 } & 3 \text { dih18 } \\ 8 \text { hcc19 } & 4 \text { dih19 } \\ 9 \text { hcc20 } & 8 \text { dih20 } \\ 9 \text { hcc21 } & 8 \text { dih21 } \\ 3 \text { hcc22 } & 4 \text { dih22 } \\ 3 \text { hcc23 } & 4 \text { dih23 }\end{array}$




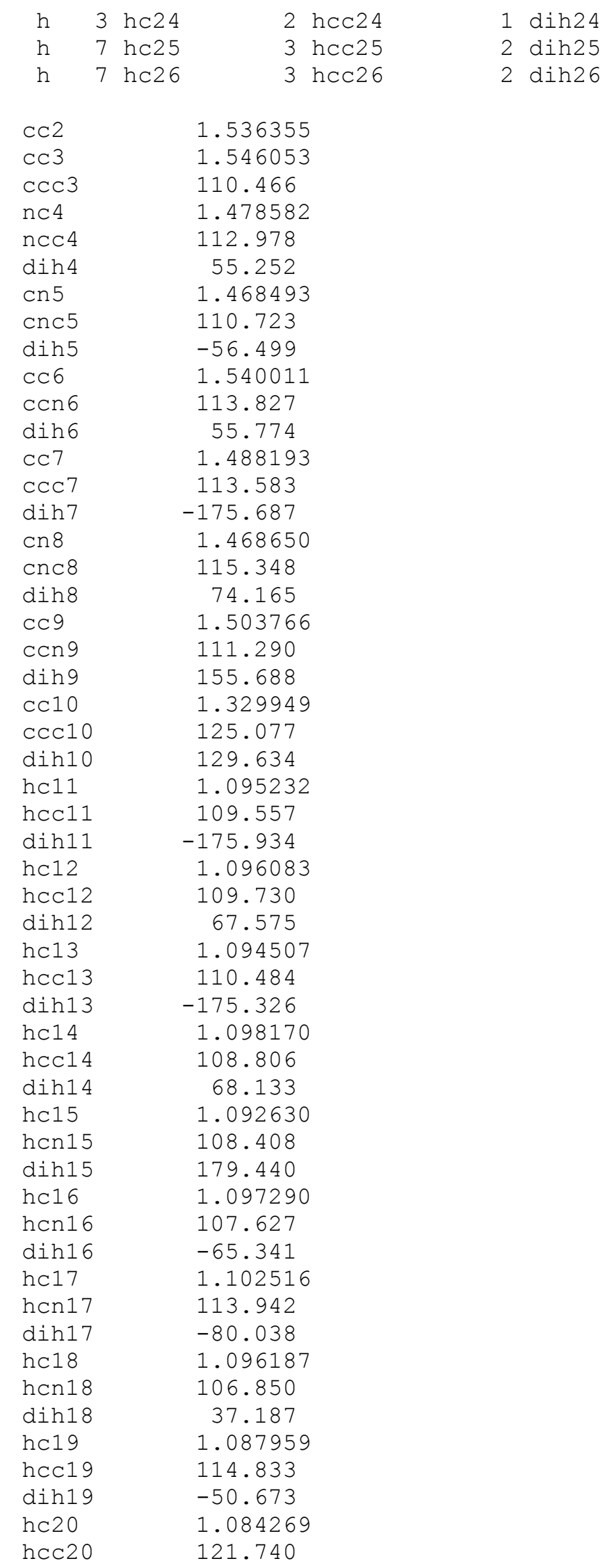




$\begin{array}{lc}\text { dih20 } & 178.325 \\ \text { hc21 } & 1.086282 \\ \text { hcc21 } & 121.555 \\ \text { dih21 } & -1.262 \\ \text { hc22 } & 1.096344 \\ \text { hcc22 } & 109.387 \\ \text { dih22 } & -66.326 \\ \text { hc23 } & 1.095424 \\ \text { hcc23 } & 109.528 \\ \text { dih23 } & 176.989 \\ \text { hc24 } & 1.107342 \\ \text { hcc24 } & 106.097 \\ \text { dih24 } & -58.277 \\ \text { hc25 } & 1.083404 \\ \text { hcc25 } & 119.613 \\ \text { dih25 } & -168.569 \\ \text { hc26 } & 1.082997 \\ \text { hcc26 } & 120.758 \\ \text { dih26 } & 22.760 \\ & \end{array}$

\section{2}

C

c $\quad 1 \quad \mathrm{Cc} 2$

n 2 nc3

C $3 \mathrm{cn} 4$

C $4 \mathrm{cc} 5$

c $5 \mathrm{cc} 6$

C $\quad 3 \mathrm{cn} 7$

C $7 \mathrm{CC} 8$

c $\quad 8$ CC 9

c $8 \mathrm{ccl} 10$

h $5 \mathrm{hcll}$

h 5 hcl2

h 6 hcl3

h $6 \mathrm{hcl} 4$

h 4 hc15

h 4 hcl 6

h 7 hc17

h 7 hcl 8

h 8 hc19

h 10 hc20

h 10 hc21

h $1 \mathrm{hc} 22$

h 1 hc23

h 2 hc24

h 9 hc25

h 9 hc26

$\begin{array}{ll}\mathrm{cc} 2 & 1.544502 \\ \mathrm{nc} 3 & 1.474142 \\ \mathrm{ncc} 3 & 113.199 \\ \mathrm{cn} 4 & 1.458484 \\ \mathrm{cnc} 4 & 115.326 \\ \text { dih4 } & -46.749 \\ \mathrm{cc5} & 1.538883 \\ \text { ccn5 } & 114.520 \\ \text { dih5 } & 48.760\end{array}$




$\begin{array}{ll}\text { cc6 } & 1.534949 \\ \text { ccc6 } & 109.891 \\ \text { dih6 } & -52.707 \\ \text { cn7 } & 1.456791 \\ \text { cnc7 } & 107.271 \\ \text { dih7 } & 87.660 \\ \text { cc8 } & 1.539930 \\ \text { ccn8 } & 102.189 \\ \text { dih8 } & 44.045 \\ \text { cc9 } & 1.560451 \\ \text { ccc9 } & 103.348 \\ \text { dih9 } & -34.698 \\ \text { cc10 } & 1.486557 \\ \text { ccc10 } & 115.241 \\ \text { dih10 } & 140.829 \\ \text { hc11 } & 1.095340 \\ \text { hcc11 } & 109.606 \\ \text { dih11 } & -174.806 \\ \text { hc12 } & 1.096616 \\ \text { hcc12 } & 110.012 \\ \text { dih12 } & 67.997 \\ \text { hc13 } & 1.094436 \\ \text { hcc13 } & 110.805 \\ \text { dih13 } & 178.091 \\ \text { hc14 } & 1.098593 \\ \text { hcc14 } & 109.193 \\ \text { dih14 } & -64.858 \\ \text { hc15 } & 1.094121 \\ \text { hcn15 } & 108.314 \\ \text { dih15 } & 173.262 \\ \text { hc16 } & 1.098855 \\ \text { hcn16 } & 107.659 \\ \text { dih16 } & -71.865 \\ \text { hc17 } & 1.094433 \\ \text { hcn17 } & 110.464 \\ \text { dih17 } & 163.956 \\ \text { hc18 } & 1.105270 \\ \text { hcn18 } & 114.357 \\ \text { dih18 } & -74.588 \\ \text { hc19 } & 1.102261 \\ \text { hcc19 } & 106.952 \\ \text { dih19 } & -97.522 \\ \text { hc20 } & 1.083968 \\ \text { hcc20 } & 120.684 \\ \text { dih20 } & 40.651 \\ \text { hc21 } & 1.084264 \\ \text { hcc21 } & 120.859 \\ \text { dih21 } & -150.935 \\ \text { hc22 } & 1.097243 \\ \text { hcc22 } & 109.427 \\ \text { dih22 } & 46.263 \\ \text { hc23 } & 1.095900 \\ \text { hcc23 } & 109.141 \\ \text { dih23 } & -69.877 \\ \text { hc24 } & 1.097831 \\ \text { hcc24 } & 111.476 \\ \text { dih24 } & 126.814 \\ & \end{array}$




$\begin{array}{ll}\text { hc25 } & 1.091679 \\ \text { hcc25 } & 111.891 \\ \text { dih25 } & -109.987 \\ \text { hc26 } & 1.093119 \\ \text { hcc26 } & 110.425 \\ \text { dih26 } & 130.457\end{array}$

13

C

C $\quad 1 \quad \mathrm{Cc} 2$

n 2 nc3

c $3 \mathrm{cn} 4$

C $4 \mathrm{CC} 5$

C $5 \mathrm{cc} 6$

C $\quad 6 \mathrm{CC} 7$

c $2 \mathrm{cc} 8$

C $6 \mathrm{CC} 9$

c $9 \mathrm{cc} 10$

C $10 \quad \mathrm{ccl} 1$

C $6 \mathrm{cc} 12$

h 5 hcl3

h 5 hcl 4

h 4 hc15

h 4 hc16

h 8 hcl 7

h 8 hcl 8

h 2 hc19

h 1 hc20

h 1 hc21

h 9 hc22

h 9 hc23

h 10 hc24

h 11 hc25

h 11 hc26

h 7 hc27

h 7 hc2 8

h 7 hc29

h 12 hc30

h 12 hc31

h 12 hc32

$\begin{array}{ll}\text { cc2 } & 1.452498 \\ \mathrm{nc} 3 & 1.476960 \\ \mathrm{ncc} 3 & 118.964 \\ \mathrm{cn} 4 & 1.463819 \\ \mathrm{cnc} 4 & 116.042 \\ \text { dih4 } & 139.505 \\ \mathrm{cc5} & 1.533144 \\ \text { ccn5 } & 113.770 \\ \text { dih5 } & -86.699 \\ \text { cc6 } & 1.554223 \\ \text { ccc6 } & 119.313 \\ \text { dih6 } & -68.788 \\ \text { cc7 } & 1.539292 \\ \text { ccc7 } & 111.328 \\ \text { dih7 } & 70.458 \\ \text { cc8 } & 1.522152\end{array}$

1 ncc3

2 cnc4 1 dih4

$3 \operatorname{ccn} 5 \quad 2$ dih5

$4 \operatorname{ccc} 6 \quad 3$ dih 6

$5 \operatorname{ccc} 7 \quad 4$ dih7

$3 \operatorname{ccn} 8 \quad 4$ dih8

$5 \operatorname{ccc} 9 \quad 4$ dih 9

$6 \operatorname{ccc} 10 \quad 5$ dihlo

$9 \operatorname{cccl1} 6$ dihl1

$5 \operatorname{ccc} 12 \quad 4$ dih12

$4 \operatorname{hccl3} 3$ dihl3

4 hec14 3 dihl4

$3 \operatorname{hen} 15 \quad 2$ dih15

$3 \operatorname{hcn} 16 \quad 2$ dih16

$2 \operatorname{hcc17} 1$ dih17

$2 \operatorname{hcc18} 1$ dih18

8 hec19 3 dih19

2 hcc20 8 dih20

$2 \operatorname{hcc} 21 \quad 8$ dih21

6 hcc22 5 dih22

6 hcc23 5 dih23

9 hcc24 6 dih24

10 hcc25 9 dih25

10 hcc26 $9 \operatorname{dih} 26$

6 hcc27 5 dih27

6 hcc28 5 dih28

6 hcc29 5 dih29

$6 \operatorname{hcc} 30 \quad 5 \operatorname{dih} 30$

6 hcc31 5 dih 31

$6 \operatorname{hcc} 32 \quad 5 \operatorname{dih} 32$ 


\begin{tabular}{|c|c|}
\hline $\operatorname{ccn} 8$ & 57.632 \\
\hline dih8 & -107.414 \\
\hline $\mathrm{CC} 9$ & 1.562272 \\
\hline $\operatorname{ccc} 9$ & 105.687 \\
\hline $\operatorname{din} 9$ & -170.663 \\
\hline 810 & 1.502850 \\
\hline C10 & 116.462 \\
\hline h10 & -178.314 \\
\hline $\operatorname{ccl1}$ & 1.330460 \\
\hline$c 11$ & 125.069 \\
\hline h11 & 118.477 \\
\hline 12 & 1.539917 \\
\hline c12 & 110.497 \\
\hline h12 & -51.719 \\
\hline 13 & 1.097490 \\
\hline c13 & 106.190 \\
\hline h13 & 169.534 \\
\hline 14 & 1.096790 \\
\hline c14 & 108.486 \\
\hline h14 & 56.048 \\
\hline 15 & 1.105091 \\
\hline n15 & 110.707 \\
\hline h15 & 34.991 \\
\hline 16 & 1.094507 \\
\hline n16 & 107.298 \\
\hline dih1 6 & 150.538 \\
\hline 17 & 1.084946 \\
\hline C17 & 119.362 \\
\hline h17 & 0.606 \\
\hline 18 & 1.089848 \\
\hline c18 & 116.404 \\
\hline dih18 & -145.418 \\
\hline 19 & 1.090183 \\
\hline c19 & 113.009 \\
\hline h19 & -106.629 \\
\hline 20 & 1.082008 \\
\hline hec20 & 120.584 \\
\hline dih20 & 129.363 \\
\hline 21 & 1.082060 \\
\hline C21 & 120.086 \\
\hline h21 & -45.405 \\
\hline 22 & 1.095982 \\
\hline hсc22 & 108.378 \\
\hline $\operatorname{dih} 22$ & -55.216 \\
\hline 23 & 1.098889 \\
\hline c23 & 107.717 \\
\hline h23 & 59.702 \\
\hline 24 & 1.088280 \\
\hline hсc24 & 116.528 \\
\hline h24 & -62.787 \\
\hline 25 & 1.084502 \\
\hline 25 & 121.723 \\
\hline h25 & 178.933 \\
\hline 26 & 1.086196 \\
\hline 26 & 121.577 \\
\hline h26 & -0.863 \\
\hline & 1.093207 \\
\hline
\end{tabular}




$\begin{array}{ll}\text { hcc27 } & 110.796 \\ \text { dih27 } & 177.706 \\ \text { hc28 } & 1.089912 \\ \text { hcc28 } & 110.807 \\ \text { dih28 } & -60.962 \\ \text { hc29 } & 1.094903 \\ \text { hcc29 } & 110.801 \\ \text { dih29 } & 58.396 \\ \text { hc30 } & 1.092610 \\ \text { hcc30 } & 111.161 \\ \text { dih30 } & -175.385 \\ \text { hc31 } & 1.095626 \\ \text { hcc31 } & 111.472 \\ \text { dih31 } & -55.018 \\ \text { hc32 } & 1.092631 \\ \text { hcc32 } & 111.239 \\ \text { dih32 } & 65.663 \\ & \end{array}$

14

C

C $\quad 1 \quad \mathrm{cc} 2$

c $2 \mathrm{cc} 3$

n 3 nc4

c $4 \mathrm{cn} 5$

C $5 \mathrm{cc} 6$

c $\quad 6 \mathrm{cc} 7$

c $7 \mathrm{CC} 8$

c $8 \mathrm{cc} 9$

c $9 \mathrm{cc} 10$

C $\quad 7 \mathrm{ccl} 1$

C $7 \mathrm{cc} 12$

h 6 hc13

h 6 hc14

h 5 hcl 5

h 5 hc16

h 3 hc17

h 3 hcl 8

h 2 hc19

h 1 hc20

h 1 hc21

h 8 hc22

h 8 hc23

h 9 hc24

h 10 hc25

h 10 hc26

h 11 hc27

h 11 hc28

h 11 hc2 9

h 12 hc30

h 12 hc31

h 12 hc32

\begin{tabular}{|c|c|c|}
\hline $1 \operatorname{ccc}^{3}$ & & \\
\hline $2 \mathrm{ncc} 4$ & 1 & $\operatorname{din} 4$ \\
\hline 3 cnc5 & 2 & $\operatorname{din} 5$ \\
\hline $4 \operatorname{ccn} 6$ & 3 & $\operatorname{din} 6$ \\
\hline $5 \operatorname{ccc} 7$ & 4 & $\operatorname{din} 7$ \\
\hline $6 \operatorname{ccc} 8$ & 5 & $\operatorname{dih} 8$ \\
\hline $7 \operatorname{ccc} 9$ & 6 & $\operatorname{din} 9$ \\
\hline $8 \operatorname{ccc} 10$ & 7 & dih10 \\
\hline $6 \operatorname{ccc} 11$ & 5 & $\operatorname{dih} 11$ \\
\hline $6 \operatorname{ccc} 12$ & 5 & $\operatorname{dih} 12$ \\
\hline 5 hсc13 & 4 & $\operatorname{dih} 13$ \\
\hline 5 hecl4 & 4 & dih14 \\
\hline $4 h \operatorname{hen} 15$ & 3 & $\operatorname{dih} 15$ \\
\hline $4 \operatorname{hcn} 16$ & 3 & $\operatorname{dih} 16$ \\
\hline $2 h \operatorname{hcc} 17$ & 1 & $\operatorname{dih} 17$ \\
\hline $2 \operatorname{hcc} 18$ & 1 & $\operatorname{dih} 18$ \\
\hline 3 hccla & 4 & dih19 \\
\hline 2 hсc20 & 3 & $\operatorname{dih} 20$ \\
\hline $2 h \mathrm{hcc} 21$ & 3 & $\operatorname{dih} 21$ \\
\hline 7 hсc22 & 6 & $\operatorname{dih} 22$ \\
\hline $7 \mathrm{hcc} 23$ & 6 & dih23 \\
\hline $8 \mathrm{hcc} 24$ & 7 & $\operatorname{dih} 24$ \\
\hline 9 hcc25 & 8 & $\operatorname{dih} 25$ \\
\hline 9 hсc26 & 8 & $\operatorname{dih} 26$ \\
\hline 7 hсc27 & 6 & $\operatorname{dih} 27$ \\
\hline 7 hcc28 & 6 & $\operatorname{dih} 28$ \\
\hline hcc29 & 6 & $\operatorname{din} 29$ \\
\hline hcc30 & 6 & $\operatorname{dih} 30$ \\
\hline hcc31 & 6 & $\operatorname{dih} 31$ \\
\hline hcc32 & 6 & dih32 \\
\hline
\end{tabular}

$\begin{array}{ll}\mathrm{cc} 2 & 1.328185 \\ \mathrm{cc} 3 & 1.503817 \\ \mathrm{ccc} 3 & 124.633 \\ \mathrm{nc} 4 & 1.444262 \\ \mathrm{ncc} 4 & 111.796\end{array}$




$\begin{array}{ll}\text { dih4 } & 132.880 \\ \text { cn5 } & 1.441331 \\ \text { cnc5 } & 112.813 \\ \text { dih5 } & -169.575 \\ \text { cc6 } & 1.533084 \\ \text { ccn6 } & 113.651 \\ \text { dih6 } & 176.961 \\ \text { cc7 } & 1.553171 \\ \text { ccc7 } & 118.309 \\ \text { dih7 } & -75.779 \\ \text { cc8 } & 1.561512 \\ \text { ccc8 } & 105.841 \\ \text { dih8 } & -177.824 \\ \text { cc9 } & 1.502952 \\ \text { ccc9 } & 116.459 \\ \text { dih9 } & 179.357 \\ \text { cc10 } & 1.330489 \\ \text { ccc10 } & 125.039 \\ \text { dih10 } & 117.824 \\ \text { cc11 } & 1.540643 \\ \text { ccc11 } & 110.352 \\ \text { dih11 } & -58.925 \\ \text { cc12 } & 1.538553 \\ \text { ccc12 } & 111.363 \\ \text { dih12 } & 63.200 \\ \text { hc13 } & 1.097223 \\ \text { hcc13 } & 107.451 \\ \text { dih13 } & 162.083 \\ \text { hc14 } & 1.095593 \\ \text { hcc14 } & 107.496 \\ \text { dih14 } & 47.805 \\ \text { hc15 } & 1.105898 \\ \text { hcn15 } & 107.841 \\ \text { dih15 } & -63.163 \\ \text { hc16 } & 1.102580 \\ \text { hcn16 } & 110.785 \\ \text { dih16 } & 50.628 \\ \text { hc17 } & 1.102746 \\ \text { hcc17 } & 110.521 \\ \text { dih17 } & 7.150 \\ \text { hc18 } & 1.107664 \\ \text { hcc18 } & 109.167 \\ \text { dih18 } & -108.381 \\ \text { hc19 } & 1.087068 \\ \text { hcc19 } & 115.005 \\ \text { dih19 } & -47.026 \\ \text { hc20 } & 1.083996 \\ \text { hcc20 } & 121.652 \\ \text { dih20 } & 179.162 \\ \text { hc21 } & 1.086290 \\ \text { hcc21 } & 121.699 \\ \text { dih21 } & -0.831 \\ \text { hc22 } & 1.095616 \\ \text { hcc22 } & 108.293 \\ \text { dih22 } & -57.507 \\ \text { hc23 } & 1.098795 \\ \text { hcc23 } & 107.678 \\ \text { } & \end{array}$




$\begin{array}{lc}\text { dih23 } & 57.372 \\ \text { hc24 } & 1.088223 \\ \text { hcc24 } & 116.543 \\ \text { dih24 } & -63.513 \\ \text { hc25 } & 1.084510 \\ \text { hcc25 } & 121.717 \\ \text { dih25 } & 178.881 \\ \text { hc26 } & 1.086164 \\ \text { hcc26 } & 121.589 \\ \text { dih26 } & -0.897 \\ \text { hc27 } & 1.092636 \\ \text { hcc27 } & 111.217 \\ \text { dih27 } & -177.650 \\ \text { hc28 } & 1.095416 \\ \text { hcc28 } & 111.451 \\ \text { dih28 } & -57.150 \\ \text { hc29 } & 1.093074 \\ \text { hcc29 } & 111.462 \\ \text { dih29 } & 63.425 \\ \text { hc30 } & 1.093137 \\ \text { hcc30 } & 110.632 \\ \text { dih30 } & 176.908 \\ \text { hc31 } & 1.091318 \\ \text { hcc31 } & 111.559 \\ \text { dih31 } & -62.178 \\ \text { hc32 } & 1.093689 \\ \text { hcc32 } & 110.985 \\ \text { dih32 } & 57.082 \\ & \end{array}$

15

C

C $\quad 1 \quad \mathrm{cc} 2$

c $2 \mathrm{cc} 3$

c $3 \mathrm{cc} 4$

n 4 nc5

C $5 \mathrm{cn} 6$

C $2 \mathrm{CC} 7$

c $2 \mathrm{cc} 8$

C 4 CC 9

C 5 cn10

C $10 \mathrm{cc} 11$

c $11 \mathrm{cc} 12$

h 1 hc13

h 1 hcl 4

h 6 hc15

h 6 hcl 6

h 10 hc17

h 10 hc18

h 11 hc19

h 12 hc20

h 12 hc21

h 3 hc22

h 3 hc23

h 4 hc24

h 9 hc25

h 9 hc26

h 8 hc27

\begin{tabular}{|c|c|c|}
\hline $\operatorname{ccc} 3$ & & \\
\hline $2 \operatorname{ccc} 4$ & 1 & dih 4 \\
\hline $3 \operatorname{ncc} 5$ & 2 & dih5 \\
\hline $4 \operatorname{cnc} 6$ & 3 & $\operatorname{dih} 6$ \\
\hline $3 \operatorname{ccc} 7$ & 4 & $\operatorname{din} 7$ \\
\hline $3 \operatorname{ccc} 8$ & 4 & dih8 \\
\hline $3 \operatorname{ccc} 9$ & 2 & dihg \\
\hline 4 cnclo & 3 & dih10 \\
\hline $5 \operatorname{ccn} 11$ & 4 & dih11 \\
\hline $0 \operatorname{ccc} 12$ & 5 & $\operatorname{dih} 12$ \\
\hline 2 hсc13 & 3 & dih13 \\
\hline 2 hccl 4 & 3 & dih14 \\
\hline 5 hen 15 & 4 & dih15 \\
\hline 5 hen16 & 4 & dih16 \\
\hline 5 hen 17 & 4 & dih17 \\
\hline $5 h \operatorname{hn} 18$ & 4 & dih18 \\
\hline 0 hcc19 & 5 & dih19 \\
\hline 1 hcc20 & 10 & $\operatorname{dih} 20$ \\
\hline 1 hсc 21 & 10 & $\operatorname{dih} 21$ \\
\hline 2 hсc 22 & 1 & $\operatorname{dih} 22$ \\
\hline 2 hсc23 & 1 & $\operatorname{dih} 23$ \\
\hline 3 hсc24 & 2 & $\operatorname{dih} 24$ \\
\hline hсc25 & 3 & $\operatorname{dih} 25$ \\
\hline $\mathrm{cc} 26$ & 3 & $\operatorname{dih} 26$ \\
\hline 27 & 3 & $\operatorname{dih} 27$ \\
\hline
\end{tabular}




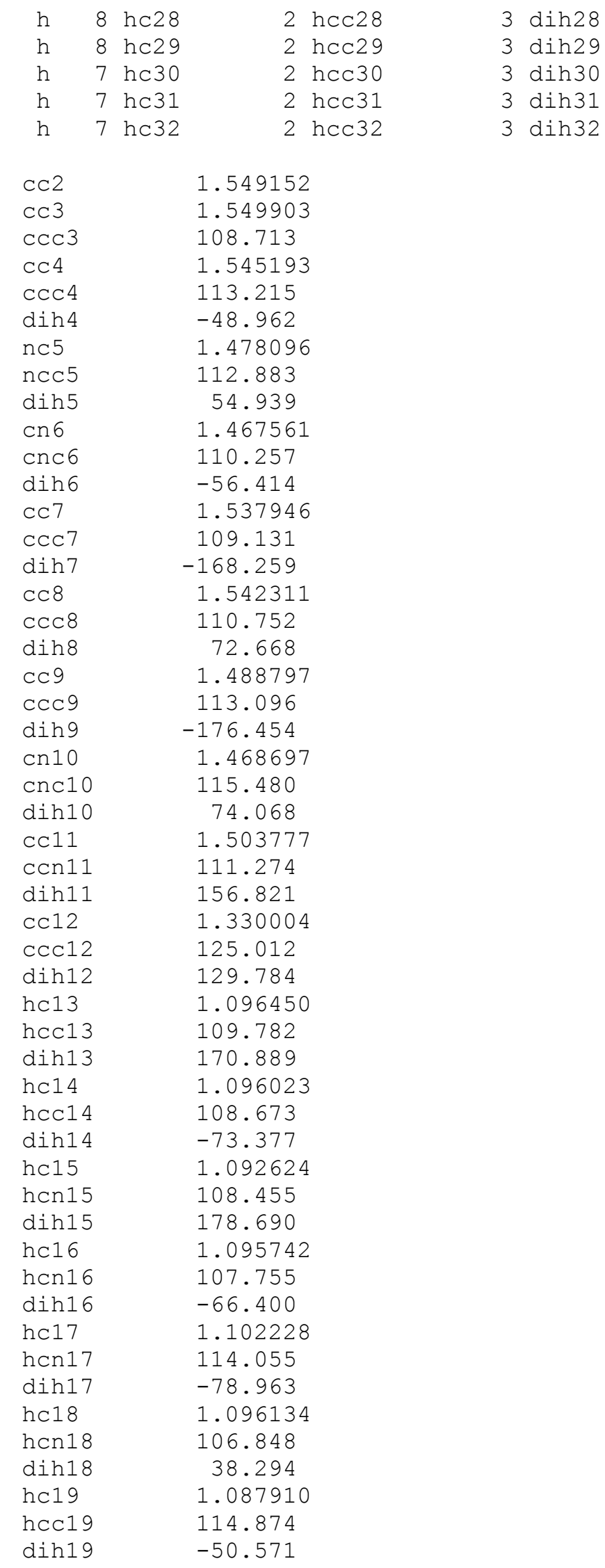




$\begin{array}{lc}\text { hc20 } & 1.084244 \\ \text { hcc20 } & 121.757 \\ \text { dih20 } & 178.288 \\ \text { hc21 } & 1.086297 \\ \text { hcc21 } & 121.525 \\ \text { dih21 } & -1.281 \\ \text { hc22 } & 1.096511 \\ \text { hcc22 } & 109.236 \\ \text { dih22 } & 72.225 \\ \text { hc23 } & 1.096599 \\ \text { hcc23 } & 109.514 \\ \text { dih23 } & -171.471 \\ \text { hc24 } & 1.104867 \\ \text { hcc24 } & 107.134 \\ \text { dih24 } & -59.185 \\ \text { hc25 } & 1.083443 \\ \text { hcc25 } & 119.540 \\ \text { dih25 } & -168.570 \\ \text { hc26 } & 1.082885 \\ \text { hcc26 } & 120.831 \\ \text { dih26 } & 22.807 \\ \text { hc27 } & 1.094426 \\ \text { hcc27 } & 110.411 \\ \text { dih27 } & 178.062 \\ \text { hc28 } & 1.092134 \\ \text { hcc28 } & 112.878 \\ \text { dih28 } & -61.746 \\ \text { hc29 } & 1.094409 \\ \text { hcc29 } & 110.699 \\ \text { dih29 } & 58.861 \\ \text { hc30 } & 1.094861 \\ \text { hcc30 } & 110.998 \\ \text { dih30 } & 179.444 \\ \text { hc31 } & 1.095039 \\ \text { hcc31 } & 111.105 \\ \text { dih31 } & -60.681 \\ \text { hc32 } & 1.094377 \\ \text { hcc32 } & 111.175 \\ \text { dih32 } & 59.396 \\ & \\ & \end{array}$

16

$\begin{array}{llll}\mathrm{C} & & & \\ \mathrm{C} & \mathrm{CC} 2\end{array}$

n 2 nc3

c $3 \mathrm{cn} 4$

C $4 \mathrm{CC} 5$

c $5 \mathrm{cc} 6$

c $3 \mathrm{cn} 7$

C $7 \mathrm{CC} 8$

c $8 \mathrm{cc} 9$

c $8 \mathrm{cc} 10$

C $6 \mathrm{ccll}$

c $6 \mathrm{cc} 12$

h 5 hcl3

h 5 hcl4

h 4 hc15

\begin{tabular}{|c|c|c|}
\hline $1 \mathrm{ncc} 3$ & & \\
\hline $2 \mathrm{cnc} 4$ & 1 & $\operatorname{dih} 4$ \\
\hline $3 \operatorname{ccn} 5$ & 2 & $\operatorname{din} 5$ \\
\hline $4 \operatorname{ccc} 6$ & 3 & $\operatorname{din} 6$ \\
\hline $2 \mathrm{cnc} 7$ & 1 & $\operatorname{din} 7$ \\
\hline $3 \operatorname{ccn} 8$ & 2 & $\operatorname{dih} 8$ \\
\hline $7 \operatorname{ccc} 9$ & 3 & $\operatorname{din} 9$ \\
\hline $9 \operatorname{ccc} 10$ & 2 & dih10 \\
\hline $5 \operatorname{ccc} 11$ & 4 & $\operatorname{dih} 11$ \\
\hline $\operatorname{ccc} 12$ & 4 & $\operatorname{dih} 12$ \\
\hline hсc13 & 3 & $\operatorname{dih} 13$ \\
\hline hec14 & 3 & $\operatorname{dih} 14$ \\
\hline hen15 & 2 & dih15 \\
\hline
\end{tabular}




\begin{tabular}{|c|c|c|c|}
\hline h & 4 hc1 6 & $3 \operatorname{hcn} 16$ & 2 \\
\hline $\mathrm{h}$ & 7 hcl 7 & $3 \operatorname{hcn} 17$ & 2 \\
\hline h & 7 hc18 & $3 \operatorname{hcn} 18$ & 2 \\
\hline h & 8 hcl 9 & 9 hсc19 & 2 \\
\hline $\mathrm{h}$ & 10 hc20 & 8 hсc 20 & 9 \\
\hline h & 10 hc21 & 8 hсc 21 & 9 \\
\hline h & 1 hс22 & 2 hсc 22 & 9 \\
\hline h & 1 hс23 & $2 \operatorname{hcc} 23$ & 9 \\
\hline h & 2 hс24 & 9 hсc24 & 8 \\
\hline h & 9 hс25 & $2 \operatorname{hcc} 25$ & 3 \\
\hline h & 9 hс26 & 2 hсc 26 & 3 \\
\hline $\mathrm{h}$ & 12 hс27 & 6 hсc 27 & 5 \\
\hline $\mathrm{h}$ & 12 hс28 & 6 hсc 28 & 5 \\
\hline h & 12 hс29 & 6 hсc2 9 & 5 \\
\hline $\mathrm{h}$ & 11 hс30 & $6 \operatorname{hcc} 30$ & 5 \\
\hline h & 11 hс31 & $6 \operatorname{hcc} 31$ & 5 \\
\hline $\mathrm{h}$ & 11 hс32 & 6 hсс 32 & 5 \\
\hline
\end{tabular}

\begin{tabular}{|c|c|}
\hline $\mathrm{cc} 2$ & 1.544802 \\
\hline nc3 & 1.474128 \\
\hline ncc 3 & 113.005 \\
\hline $\mathrm{Cn} 4$ & 1.458482 \\
\hline Cnc4 & 114.907 \\
\hline dih4 & -47.174 \\
\hline $\operatorname{cc} 5$ & 1.539004 \\
\hline $\operatorname{ccn} 5$ & 114.501 \\
\hline dih5 & 48.606 \\
\hline $\operatorname{cc} 6$ & 1.547931 \\
\hline $\operatorname{ccc} 6$ & 112.679 \\
\hline $\operatorname{dih} 6$ & -52.108 \\
\hline $\mathrm{cn} 7$ & 1.457766 \\
\hline Cnc 7 & 107.133 \\
\hline $\operatorname{dih} 7$ & 86.417 \\
\hline $\operatorname{cc} 8$ & 1.539830 \\
\hline $\operatorname{ccn} 8$ & 102.270 \\
\hline dih8 & 44.134 \\
\hline $\operatorname{cc} 9$ & 1.561049 \\
\hline $\operatorname{ccc} 9$ & 103.397 \\
\hline dihg & -34.309 \\
\hline $\mathrm{CC} 10$ & 1.486544 \\
\hline $\operatorname{ccc} 10$ & 115.184 \\
\hline $\operatorname{dih} 10$ & 140.065 \\
\hline $\mathrm{CC} 11$ & 1.537685 \\
\hline $\operatorname{ccc} 11$ & 109.357 \\
\hline $\operatorname{dih} 11$ & 170.925 \\
\hline cc12 & 1.541873 \\
\hline $\operatorname{ccc} 12$ & 111.041 \\
\hline $\operatorname{dih} 12$ & -69.488 \\
\hline hc13 & 1.096769 \\
\hline hecl 3 & 109.530 \\
\hline $\operatorname{dih} 13$ & -174.915 \\
\hline he14 & 1.096606 \\
\hline $\operatorname{hccl} 4$ & 109.341 \\
\hline $\operatorname{dih} 14$ & 68.713 \\
\hline & 1.094151 \\
\hline 15 & 108.290 \\
\hline 15 & 172.022 \\
\hline
\end{tabular}




$\begin{array}{lc}\text { hc16 } & 1.096644 \\ \text { hcn16 } & 107.832 \\ \text { dih16 } & -73.506 \\ \text { hc17 } & 1.094358 \\ \text { hcn17 } & 110.429 \\ \text { dih17 } & 164.089 \\ \text { hc18 } & 1.105058 \\ \text { hcn18 } & 114.358 \\ \text { dih18 } & -74.448 \\ \text { hc19 } & 1.102227 \\ \text { hcc19 } & 106.940 \\ \text { dih19 } & -98.319 \\ \text { hc20 } & 1.083936 \\ \text { hcc20 } & 120.655 \\ \text { dih20 } & 40.517 \\ \text { hc21 } & 1.084278 \\ \text { hcc21 } & 120.867 \\ \text { dih21 } & -151.126 \\ \text { hc22 } & 1.097176 \\ \text { hcc22 } & 108.727 \\ \text { dih22 } & 46.246 \\ \text { hc23 } & 1.097165 \\ \text { hcc23 } & 109.177 \\ \text { dih23 } & -69.320 \\ \text { hc24 } & 1.095744 \\ \text { hcc24 } & 110.992 \\ \text { dih24 } & 127.611 \\ \text { hc25 } & 1.091648 \\ \text { hcc25 } & 111.795 \\ \text { dih25 } & -109.040 \\ \text { hc26 } & 1.092973 \\ \text { hcc26 } & 110.553 \\ \text { dih26 } & 131.415 \\ \text { hc27 } & 1.094577 \\ \text { hcc27 } & 110.470 \\ \text { dih27 } & -179.689 \\ \text { hc28 } & 1.094539 \\ \text { hcc28 } & 110.602 \\ \text { dih28 } & -60.569 \\ \text { hc29 } & 1.091863 \\ \text { hcc29 } & 112.827 \\ \text { dih29 } & 59.959 \\ \text { hc30 } & 1.094940 \\ \text { hcc30 } & 111.041 \\ \text { dih30 } & -178.586 \\ \text { hc31 } & 1.094353 \\ \text { hcc31 } & 111.151 \\ \text { dih31 } & -58.547 \\ \text { hc32 } & 1.094981 \\ \text { hcc32 } & 111.032 \\ \text { dih32 } & 61.505 \\ & \end{array}$

transiton state 5 to 6

C

c $\quad 1 \quad \mathrm{Cc} 2$

n 2 nc3 1 ncc3

C $3 \mathrm{cn} 4 \quad 2$ cnc4 1 dih 4 


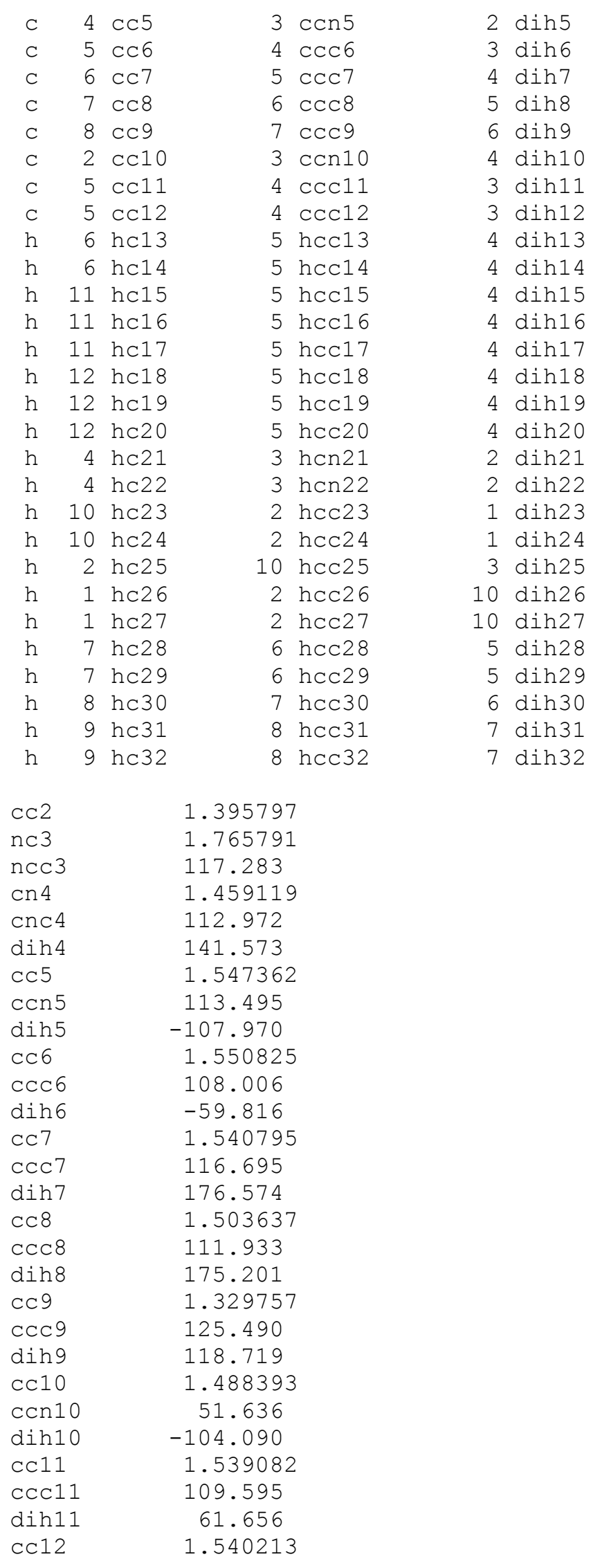




\begin{tabular}{|c|c|}
\hline $\operatorname{ccc} 12$ & 107.295 \\
\hline $\operatorname{dih} 12$ & -179.447 \\
\hline hc13 & 1.098318 \\
\hline hcc13 & 107.912 \\
\hline $\operatorname{dih} 13$ & -60.957 \\
\hline hc14 & 1.092936 \\
\hline hec14 & 108.136 \\
\hline $\operatorname{dih} 14$ & 53.495 \\
\hline 15 & 1.095285 \\
\hline cc15 & 110.675 \\
\hline h15 & 55.997 \\
\hline 16 & 1.092879 \\
\hline hcc16 & 111.325 \\
\hline $\ln 16$ & 175.113 \\
\hline 17 & 1.091899 \\
\hline$c 17$ & 111.100 \\
\hline h17 & -63.948 \\
\hline 18 & 1.094829 \\
\hline hcc18 & 110.933 \\
\hline hin 8 & -59.258 \\
\hline 19 & 1.094921 \\
\hline cc19 & 111.291 \\
\hline h19 & 60.762 \\
\hline$h c 20$ & 1.092539 \\
\hline hcc20 & 111.580 \\
\hline $\operatorname{din} 20$ & -178.787 \\
\hline hc21 & 1.103210 \\
\hline hen21 & 112.577 \\
\hline h21 & 16.788 \\
\hline hc22 & 1.102160 \\
\hline hen22 & 106.619 \\
\hline $\ln 22$ & 132.612 \\
\hline 23 & 1.089331 \\
\hline 23 & 116.128 \\
\hline $\operatorname{din} 23$ & -10.432 \\
\hline hc24 & 1.093200 \\
\hline hсc24 & 116.670 \\
\hline $\ln 24$ & -146.875 \\
\hline 25 & 1.083213 \\
\hline 25 & 115.846 \\
\hline $\operatorname{din} 25$ & -91.252 \\
\hline hс26 & 1.082399 \\
\hline hсc26 & 120.775 \\
\hline $\operatorname{din} 26$ & 178.109 \\
\hline 27 & 1.083015 \\
\hline$c 27$ & 121.126 \\
\hline $\operatorname{din} 27$ & -6.550 \\
\hline hc28 & 1.093863 \\
\hline$c 28$ & 110.392 \\
\hline h28 & -62.966 \\
\hline 29 & 1.097426 \\
\hline 29 & 109.747 \\
\hline h29 & 54.543 \\
\hline 30 & 1.089816 \\
\hline & 115.696 \\
\hline 30 & -60.579 \\
\hline & 1.084387 \\
\hline
\end{tabular}




$\begin{array}{lc}\text { hсc31 } & 121.726 \\ \text { dih31 } & -179.592 \\ \text { hc32 } & 1.086208 \\ \text { hcc32 } & 121.556 \\ \text { dih32 } & 0.433\end{array}$

transition state 6 to 7

C

C $\quad 1 \quad \mathrm{cc} 2$

c $2 \mathrm{cc} 3$

n 3 nc4

c 4 cn 5

c $5 \mathrm{cc} 6$

C $\quad 6 \mathrm{cc} 7$

C $\quad 6 \mathrm{cc} 8$

c $6 \mathrm{cc} 9$

c $9 \mathrm{cc} 10$

C $\quad 10 \quad \operatorname{cc} 11$

C $11 \mathrm{cc} 12$

h 9 hc13

h 9 hc14

h 7 hc15

h 7 hcl 6

h 7 hc17

h 8 hcl 8

h 8 hc19

h 8 hc20

h 5 hc21

h 5 hc22

h 3 hc23

h 3 hc24

h 2 hc25

h 1 hc26

h 1 hc27

h 10 hc28

h 10 hc29

h 11 hc30

h 12 hc31

h 12 hc32
$1 \operatorname{ccc} 3$

2 ncc 4

3 cnc5

$4 \operatorname{ccn} 6$

$5 \operatorname{ccc} 7$

$5 \operatorname{ccc} 8$

$5 \operatorname{ccc} 9$

$6 \operatorname{ccc} 10$

$9 \operatorname{ccc} 11$

$10 \operatorname{ccc} 12$

6 hcc13

6 hcc14

6 hcc15

6 hccl 6

6 hcc17

6 hcc18

6 hec19

6 hcc 20

4 hen 21

4 hen 22

2 hcc23

2 hcc 24

3 hcc 25

2 hcc 26

2 hcc27

9 hcc28

9 hcc 29

10 hcc 30

11 hcc 31

11 hcc 32
$1 \operatorname{dih} 4$

2 dih 5

3 dih 6

4 dih 7

4 dih 8

4 dih9

5 dih 10

6 dih 11

9 dih 12

5 dih13

5 dih14

5 dih15

5 dih16

5 dih17

5 dih 18

5 dih 19

5 dih20

3 dih21

3 dih22

1 dih23

1 dih24

4 dih25

3 dih 26

3 dih27

6 dih 28

$6 \operatorname{din} 29$

9 dih 30

10 dih 31

10 dih 32

$\begin{array}{ll}\text { cc2 } & 1.328829 \\ \text { cc3 } & 1.507240 \\ \text { ccc3 } & 124.565 \\ \text { nc4 } & 1.463260 \\ \text { ncc4 } & 110.059 \\ \text { dih4 } & 130.663 \\ \text { cn5 } & 1.453453 \\ \text { cnc5 } & 115.703 \\ \text { dih5 } & -145.491 \\ \text { cc6 } & 1.560043 \\ \text { ccn6 } & 121.517 \\ \text { dih6 } & -71.354 \\ \text { cc7 } & 1.540693 \\ \text { ccc7 } & 113.103 \\ \operatorname{dih} 7 & 68.073 \\ \operatorname{cc} 8 & 1.540621\end{array}$




$\begin{array}{ll}\text { ccc8 } & 107.012 \\ \text { dih8 } & -173.142 \\ \text { cc9 } & 1.541606 \\ \text { ccc9 } & 108.972 \\ \text { dih9 } & -55.694 \\ \text { cc10 } & 1.534277 \\ \text { ccc10 } & 115.697 \\ \text { dih10 } & 54.678 \\ \text { cc11 } & 1.517699 \\ \text { ccc11 } & 114.962 \\ \text { dih11 } & -65.072 \\ \text { cc12 } & 1.379123 \\ \text { ccc12 } & 119.914 \\ \text { dih12 } & 172.806 \\ \text { hc13 } & 1.096210 \\ \text { hcc13 } & 108.870 \\ \text { dih13 } & 177.677 \\ \text { hc14 } & 1.097743 \\ \text { hcc14 } & 108.544 \\ \text { dih14 } & -67.277 \\ \text { hc15 } & 1.094863 \\ \text { hcc15 } & 109.953 \\ \text { dih15 } & 173.682 \\ \text { hc16 } & 1.090199 \\ \text { hcc16 } & 113.131 \\ \text { dih16 } & -66.246 \\ \text { hc17 } & 1.094198 \\ \text { hcc17 } & 111.009 \\ \text { dih17 } & 54.876 \\ \text { hc18 } & 1.094621 \\ \text { hcc18 } & 111.113 \\ \text { dih18 } & -62.645 \\ \text { hc19 } & 1.094042 \\ \text { hcc19 } & 111.371 \\ \text { dih19 } & 57.644 \\ \text { hc20 } & 1.094287 \\ \text { hcc20 } & 110.823 \\ \text { dih20 } & 177.659 \\ \text { hc21 } & 1.101563 \\ \text { hcn21 } & 106.887 \\ \text { dih21 } & 53.304 \\ \text { hc22 } & 1.097507 \\ \text { hcn22 } & 106.425 \\ \text { dih22 } & 166.635 \\ \text { hc23 } & 1.097426 \\ \text { hcc23 } & 109.298 \\ \text { dih23 } & 9.387 \\ \text { hc24 } & 1.100742 \\ \text { hcc24 } & 109.203 \\ \text { dih24 } & -105.690 \\ \text { hc25 } & 1.086359 \\ \text { hcc25 } & 115.387 \\ \text { dih25 } & -49.080 \\ \text { hc26 } & 1.084353 \\ \text { hcc26 } & 121.739 \\ \text { dih26 } & 179.425 \\ \text { hc27 } & 1.086538 \\ & \\ & \end{array}$




$\begin{array}{lc}\text { hcc27 } & 121.667 \\ \text { dih27 } & -0.658 \\ \text { hc28 } & 1.095744 \\ \text { hcc28 } & 110.990 \\ \text { dih28 } & 59.914 \\ \text { hc29 } & 1.096583 \\ \text { hcc29 } & 107.873 \\ \text { dih29 } & 175.020 \\ \text { hc30 } & 1.086146 \\ \text { hcc30 } & 115.352 \\ \text { dih30 } & -39.421 \\ \text { hc31 } & 1.083114 \\ \text { hcc31 } & 121.200 \\ \text { dih31 } & 165.151 \\ \text { hc32 } & 1.085114 \\ \text { hcc32 } & 121.419 \\ \text { dih32 } & -20.431\end{array}$

\section{transition state 6 to 7}

C

C $\quad 1 \quad \mathrm{CC} 2$

c 2 cc3

c $3 \mathrm{cc} 4$

c 4 Cc5

n 5 nc 6

c $\quad 2 \quad \mathrm{CC} 7$

c $\quad 2$ CC 8

c 5 cc 9

C $6 \mathrm{cn} 10$

C $10 \quad \mathrm{CC} 11$

c $11 \mathrm{cc} 12$

h 3 hc13

h 3 hel4

h 8 hc15

h 8 hc16

h 8 hcl 7

h 7 hcl 8

h 7 hc19

h 7 hc20

h 1 hc21

h 1 hc22

h 10 hc23

h 10 hc24

h 11 hc25

h 12 hc26

h 12 hc27

h 4 hc28

h 4 hc29

h 5 hc30

h 9 hc31

h 9 hc32
$1 \operatorname{ccc} 3$

$2 \operatorname{ccc} 4$

$3 \operatorname{ccc} 5$

4 nec 6

$3 \operatorname{ccc} 7$

$3 \operatorname{ccc} 8$

$4 \operatorname{ccc} 9$

5 enc10

$6 \operatorname{ccn} 11$

$10 \operatorname{ccc} 12$

2 hccl3

2 hecl 4

2 hcc15

2 hcc16

2 hccl 7

2 hccl 8

2 hcc19

2 hcc20

2 hcc21

2 hcc22

$6 \mathrm{hcn} 23$

$6 \mathrm{hcn} 24$

10 hcc 25

11 hcc 26

11 hcc27

3 hcc28

3 hcc 29

4 hcc 30

5 hcc 31

5 hcc 32
$1 \operatorname{din} 4$

2 dih5

3 dih 6

4 dih 7

4 dih8

3 dih 9

4 dih10

5 dih 11

6 dih 12

$1 \operatorname{dih} 13$

1 dih14

$3 \operatorname{dih} 15$

3 dih16

3 dih17

3 dih18

3 dih19

3 dih20

$3 \operatorname{dih} 21$

3 dih22

5 dih23

5 dih 24

6 dih 25

10 dih 26

10 dih 27

$2 \operatorname{din} 28$

2 dih29

$3 \operatorname{din} 30$

4 dih 31

4 dih 32

$\begin{array}{ll}\mathrm{CC} 2 & 1.558132 \\ \mathrm{cc} 3 & 1.548589 \\ \mathrm{ccc} 3 & 108.198 \\ \mathrm{cc} 4 & 1.535575\end{array}$




\begin{tabular}{|c|c|}
\hline $\operatorname{CcC} 4$ & 113.433 \\
\hline dih4 & 48.933 \\
\hline $\operatorname{cc} 5$ & 1.541381 \\
\hline $\operatorname{ccc} 5$ & 110.518 \\
\hline dih5 & -54.854 \\
\hline nc 6 & 1.478132 \\
\hline $\mathrm{ncc} 6$ & 111.911 \\
\hline dih 6 & 55.406 \\
\hline $\mathrm{CC} 7$ & 1.538102 \\
\hline CC 7 & 109.510 \\
\hline dih 7 & 166.619 \\
\hline $\operatorname{cc} 8$ & 1.541143 \\
\hline $\operatorname{ccc} 8$ & 110.566 \\
\hline dih8 & -74.239 \\
\hline $\operatorname{cc} 9$ & 1.500361 \\
\hline $\operatorname{cc} 9$ & 113.709 \\
\hline h9 & 179.620 \\
\hline 10 & 1.457246 \\
\hline enclo & 112.469 \\
\hline dih10 & 83.843 \\
\hline 11 & 1.508666 \\
\hline cn11 & 110.211 \\
\hline $\operatorname{dih} 11$ & 55.750 \\
\hline $\mathrm{cc} 12$ & 1.362092 \\
\hline $\operatorname{ccc} 12$ & 122.839 \\
\hline dih12 & -147.914 \\
\hline hc13 & 1.096179 \\
\hline cc13 & 109.610 \\
\hline dih13 & 173.030 \\
\hline hc14 & 1.098173 \\
\hline hcc14 & 108.433 \\
\hline hin14 & -71.409 \\
\hline 15 & 1.095014 \\
\hline$=15$ & 110.269 \\
\hline dih15 & -66.200 \\
\hline hc16 & 1.090672 \\
\hline hcc16 & 112.838 \\
\hline in1 6 & 53.815 \\
\hline 17 & 1.094681 \\
\hline$=17$ & 110.964 \\
\hline dih17 & 174.721 \\
\hline hc18 & 1.095186 \\
\hline hcc18 & 111.193 \\
\hline $\operatorname{dih} 18$ & 179.682 \\
\hline 19 & 1.094311 \\
\hline hec19 & 111.331 \\
\hline dih19 & -60.055 \\
\hline hc20 & 1.094522 \\
\hline hcc20 & 110.900 \\
\hline $\operatorname{dih} 20$ & 59.992 \\
\hline hс21 & 1.095264 \\
\hline 21 & 109.827 \\
\hline $\operatorname{dih} 21$ & -172.065 \\
\hline 22 & 1.098812 \\
\hline & 107.172 \\
\hline 22 & 72.571 \\
\hline & 1.09683 \\
\hline
\end{tabular}




$\begin{array}{lc}\text { hcn23 } & 107.384 \\ \text { dih23 } & 174.617 \\ \text { hc24 } & 1.102822 \\ \text { hcn24 } & 115.059 \\ \text { dih24 } & -66.995 \\ \text { hc25 } & 1.086254 \\ \text { hcc25 } & 114.810 \\ \text { dih25 } & 54.137 \\ \text { hc26 } & 1.083359 \\ \text { hcc26 } & 121.514 \\ \text { dih26 } & -168.799 \\ \text { hc27 } & 1.085336 \\ \text { hcc27 } & 121.339 \\ \text { dih27 } & 15.530 \\ \text { hc28 } & 1.095889 \\ \text { hcc28 } & 110.916 \\ \text { dih28 } & 66.938 \\ \text { hc29 } & 1.095323 \\ \text { hcc29 } & 109.812 \\ \text { dih29 } & -175.732 \\ \text { hc30 } & 1.102107 \\ \text { hcc30 } & 107.204 \\ \text { dih30 } & -59.828 \\ \text { hc31 } & 1.083837 \\ \text { hcc31 } & 118.505 \\ \text { dih31 } & 148.139 \\ \text { hc32 } & 1.083882 \\ \text { hcc32 } & 119.001 \\ \text { dih32 } & -1.196\end{array}$

transition state 7 to 8

$\mathrm{C}$

C $\quad 1 \quad \mathrm{CC} 2$

C $2 \mathrm{CC} 3 \quad 1 \mathrm{CCC} 3$

c $3 \mathrm{CC} 4 \quad 2 \operatorname{ccC} 4$

c $4 \operatorname{cc} 5 \quad 3 \operatorname{ccc} 5$

$\mathrm{n} \quad 5$ nc6 4 ncc 6

$\begin{array}{llll}\text { C } & 2 \mathrm{CC} 7 & 3 \mathrm{CCC} 7\end{array}$

C $2 \mathrm{cc} 8 \quad 3 \mathrm{ccC} 8$

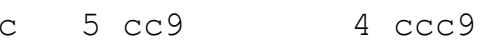

C $6 \mathrm{cnl0} \quad 5 \mathrm{cncl0}$

c $10 \mathrm{cc} 11 \quad 6 \mathrm{ccn} 11$

C $11 \mathrm{cc} 12 \quad 10 \quad \mathrm{ccc} 12$

h 3 hc13 2 hcc13

h 3 hcl4 2 hccl4

h 8 hcl5 2 hcc15

h 8 hc16 2 hcc16

h 8 hc17 2 hcc17

h 7 hcl 2 hcc18

h 7 hc19 2 hcc19

h 7 hc20 2 hcc 20

h 1 hc21 2 hcc21

h 1 hc22 2 hcc22

h 10 hc23 6 hcn23

h 10 hc24 6 hcn 24

h 11 hc25 10 hcc25

h 12 hc26

10 hcc25
11 hcc 26

1 dih4

2 dih5

3 dih 6

4 dih 7

4 dih8

3 dih 9

4 dih 10

5 dih 11

6 dih 12

1 dih13

1 dih14

3 dih15

3 dih16

3 dih17

3 dih18

3 dih19

3 dih 20

3 dih21

3 dih22

5 dih23

5 dih 24

6 dih 25

$10 \operatorname{din} 26$ 


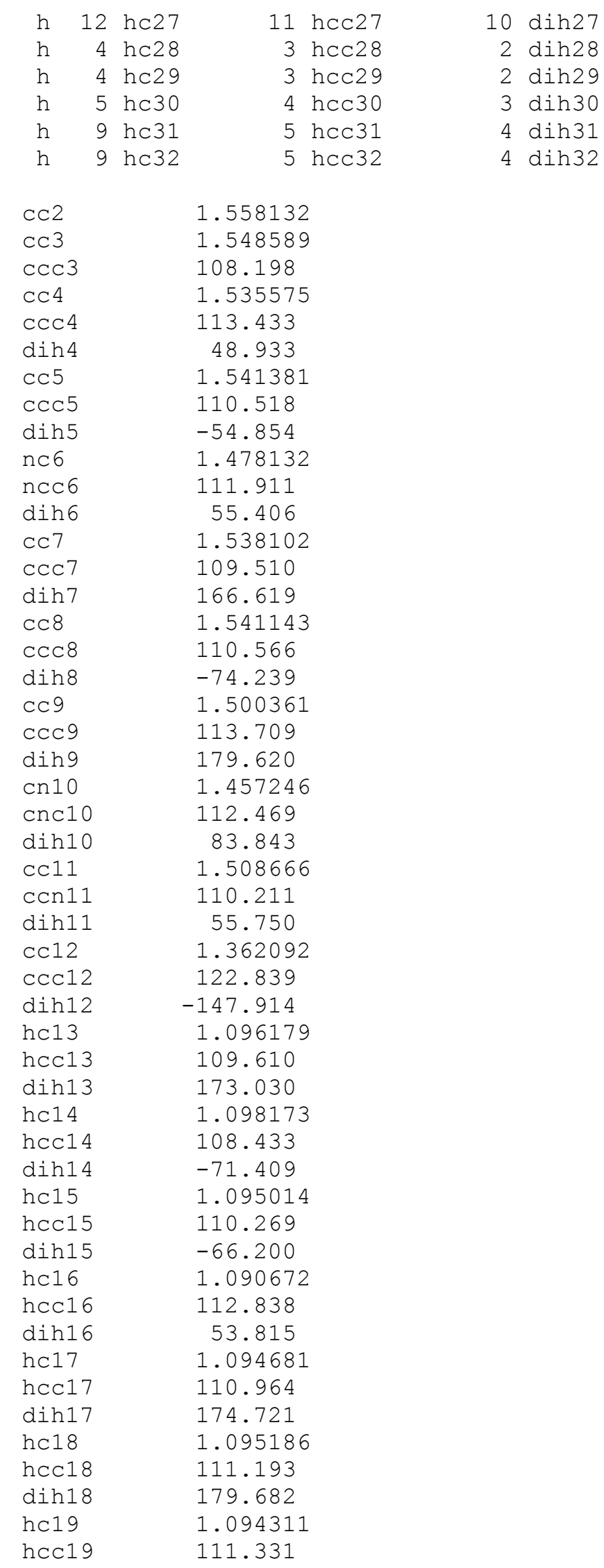




\begin{tabular}{lc} 
dih19 & -60.055 \\
hc20 & 1.094522 \\
hcc20 & 110.900 \\
dih20 & 59.992 \\
hc21 & 1.095264 \\
hcc21 & 109.827 \\
dih21 & -172.065 \\
hc22 & 1.098812 \\
hcc22 & 107.172 \\
dih22 & 72.571 \\
hc23 & 1.096834 \\
hcn23 & 107.384 \\
dih23 & 174.617 \\
hc24 & 1.102822 \\
hcn24 & 115.059 \\
dih24 & -66.995 \\
hc25 & 1.086254 \\
hcc25 & 114.810 \\
dih25 & 54.137 \\
hc26 & 1.083359 \\
hcc26 & 121.514 \\
dih26 & -168.799 \\
hc27 & 1.085336 \\
hcc27 & 121.339 \\
dih27 & 15.530 \\
hc28 & 1.095889 \\
hcc28 & 110.916 \\
dih28 & 66.938 \\
hc29 & 1.095323 \\
hcc29 & 109.812 \\
dih29 & -175.732 \\
hc30 & 1.102107 \\
hcc30 & 107.204 \\
dih30 & -59.828 \\
hc31 & 1.083837 \\
hcc31 & 118.505 \\
dih31 & 148.139 \\
hc32 & 1.083882 \\
hcc32 & 119.001 \\
dih32 & -1.196 \\
& \\
\hline &
\end{tabular}

transition state 9 to 10

C

c $\quad 1 \quad \mathrm{cc} 2$

n 2 nc3

c $3 \mathrm{cn} 4$

c 4 CC5

c $5 \mathrm{cc} 6$

C $\quad 6 \mathrm{CC} 7$

c $\quad 7 \quad \mathrm{cc} 8$

c $8 \mathrm{cc} 9$

c $2 \operatorname{cc} 10$

h 5 hcll

h 5 hc12

h 6 hc13

h 6 hc14

h 4 hc15

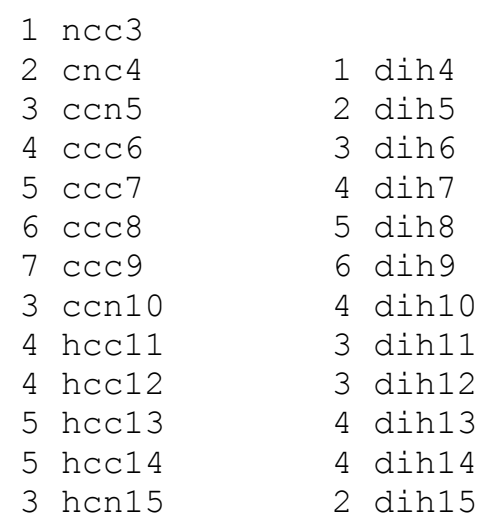




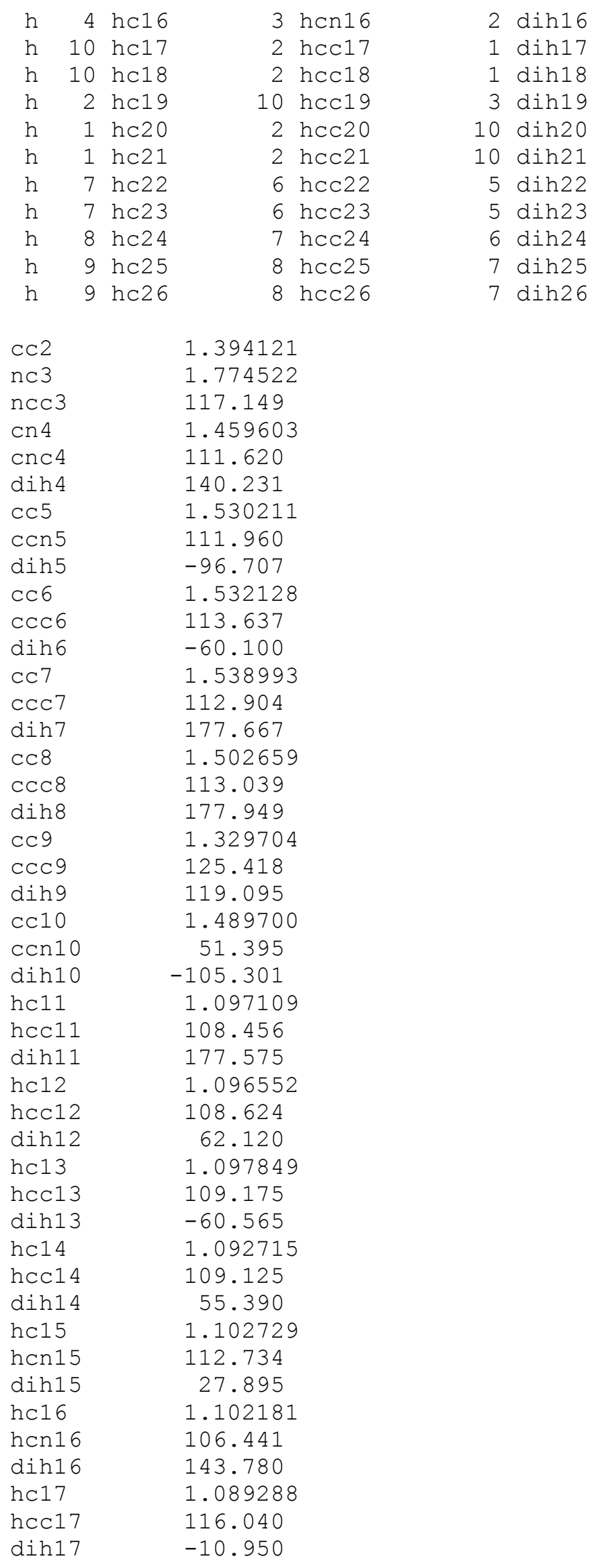




$\begin{array}{lc}\text { hc18 } & 1.093370 \\ \text { hcc18 } & 116.562 \\ \text { dih18 } & -147.063 \\ \text { hc19 } & 1.083647 \\ \text { hcc19 } & 115.786 \\ \text { dih19 } & -91.025 \\ \text { hc20 } & 1.082399 \\ \text { hcc20 } & 120.789 \\ \text { dih20 } & 178.789 \\ \text { hc21 } & 1.082974 \\ \text { hcc21 } & 121.114 \\ \text { dih21 } & -6.119 \\ \text { hc22 } & 1.095716 \\ \text { hcc22 } & 109.665 \\ \text { dih22 } & -59.492 \\ \text { hc23 } & 1.099141 \\ \text { hcc23 } & 108.744 \\ \text { dih23 } & 56.687 \\ \text { hc24 } & 1.089740 \\ \text { hcc24 } & 115.722 \\ \text { dih24 } & -60.179 \\ \text { hc25 } & 1.084353 \\ \text { hcc25 } & 121.766 \\ \text { dih25 } & -179.541 \\ \text { hc26 } & 1.086251 \\ \text { hcc26 } & 121.508 \\ \text { dih26 } & 0.520\end{array}$

transition state 10 to 11

$\mathrm{C}$

$\begin{array}{lll}\text { c } & 1 & \mathrm{CC} 2\end{array}$

c 2 cc 3

C 4 cn5 3 cnc5

c $5 \operatorname{cc} 6 \quad 4 \operatorname{ccn} 6$

$\begin{array}{ll}5 \mathrm{cc} 7 & 5 \mathrm{CCC} 7\end{array}$

$\begin{array}{ll}\text { C } \quad 7 \mathrm{CC} 8 & 6 \mathrm{cCC} 8\end{array}$

$8 \mathrm{CC} 9$

c $9 \mathrm{cc} 10$

h 6 hcll

$7 \operatorname{ccc} 9$

$8 \operatorname{ccc} 10$

5 hecl1

5 hec12

6 hcc13

6 hec14

$4 \mathrm{hcn} 15$

$4 \mathrm{hcn} 16$

$2 \operatorname{hcc} 17$

2 hcc18

3 hec19

2 hcc20

2 hcc21

7 hcc22

7 hcc23

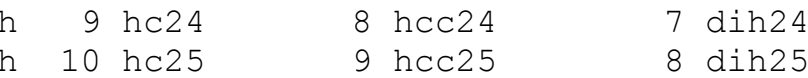

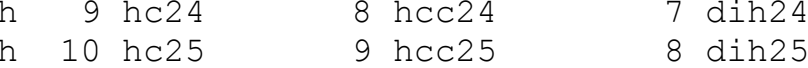

$1 \operatorname{din} 4$

2 dih5

3 dih 6

4 dih7

5 dih 8

6 dih 9

7 dih10

4 dih11

4 dih12

5 dih13

5 dih14

3 dih15

3 dih16

1 dih17

1 dih18

4 dih19

$3 \operatorname{dih} 20$

3 dih21

6 dih22

6 dih23

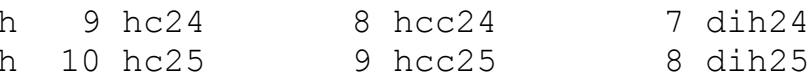

h 10 hc26

9 hcc26

8 dih 26 


$\begin{array}{ll}\text { cc2 } & 1.332410 \\ \text { cc3 } & 1.507019 \\ \text { ccc3 } & 124.432 \\ \text { nc4 } & 1.463407 \\ \text { ncc4 } & 110.509 \\ \text { dih4 } & 130.452 \\ \text { cn5 } & 1.454975 \\ \text { cnc5 } & 112.493 \\ \text { dih5 } & -153.798 \\ \text { cc6 } & 1.540581 \\ \text { ccn6 } & 117.626 \\ \text { dih6 } & -62.229 \\ \text { cc7 } & 1.531799 \\ \text { ccc7 } & 112.370 \\ \text { dih7 } & -63.960 \\ \text { cc8 } & 1.536210 \\ \text { ccc8 } & 114.593 \\ \text { dih8 } & 59.089 \\ \text { cc9 } & 1.520149 \\ \text { ccc9 } & 116.123 \\ \text { dih9 } & -63.606 \\ \text { cc10 } & 1.355395 \\ \text { ccc10 } & 120.931 \\ \text { dih10 } & 169.272 \\ \text { hc11 } & 1.098278 \\ \text { hcc11 } & 109.979 \\ \text { dih11 } & 58.885 \\ \text { hc12 } & 1.097750 \\ \text { hcc12 } & 108.320 \\ \text { dih12 } & 174.980 \\ \text { hc13 } & 1.097584 \\ \text { hcc13 } & 109.437 \\ \text { dih13 } & -178.717 \\ \text { hc14 } & 1.100196 \\ \text { hcc14 } & 108.892 \\ \text { dih14 } & -62.961 \\ \text { hc15 } & 1.103825 \\ \text { hcn15 } & 106.942 \\ \text { dih15 } & 60.718 \\ \text { hc16 } & 1.099520 \\ \text { hcn16 } & 107.622 \\ \text { dih16 } & 174.877 \\ \text { hc17 } & 1.102795 \\ \text { hcc17 } & 109.528 \\ \text { dih17 } & 8.391 \\ \text { hc18 } & 1.103914 \\ \text { hcc18 } & 109.554 \\ \text { dih18 } & -106.490 \\ \text { hc19 } & 1.088474 \\ \text { hcc19 } & 115.406 \\ \text { dih19 } & -49.574 \\ \text { hc20 } & 1.086805 \\ \text { hcc20 } & 121.859 \\ \text { dih20 } & 179.173 \\ \text { hc21 } & 1.088766 \\ \text { hcc21 } & 121.764 \\ \text { dih21 } & -0.843 \\ & \end{array}$




$\begin{array}{ll}\text { hc22 } & 1.099436 \\ \text { hcc22 } & 109.864 \\ \text { dih22 } & 61.173 \\ \text { hc23 } & 1.099269 \\ \text { hcc23 } & 107.911 \\ \text { dih23 } & 175.826 \\ \text { hc24 } & 1.086923 \\ \text { hcc24 } & 116.246 \\ \text { dih24 } & -35.582 \\ \text { hc25 } & 1.085920 \\ \text { hcc25 } & 121.461 \\ \text { dih25 } & 168.523 \\ \text { hc26 } & 1.088032 \\ \text { hcc26 } & 121.646 \\ \text { dih26 } & -16.346\end{array}$

transition state 11 to 12

\section{n}

C $1 \mathrm{cn} 2$

C $2 \mathrm{cc} 3$

c $3 \mathrm{cc} 4$

c $4 \mathrm{CC} 5$

C $5 \mathrm{cc} 6$

C $\quad 6 \mathrm{CC} 7$

C $1 \mathrm{cn} 8$

c $8 \mathrm{cc} 9$

c $9 \mathrm{cc} 10$

h 3 hcll

h $3 \mathrm{hcl} 2$

h 4 hc13

h 4 hc14

h 2 hc15

h 2 hc16

h 8 hc17

h 8 hcl 8

h 9 hcl 9

h 10 hc20

h 10 hc21

h 5 hc22

h 5 hc23

h 6 hc24

h 7 hc25

h 7 hc26

cn2

$\mathrm{cc} 3$

$\mathrm{ccn} 3$

$\mathrm{CC} 4$

$\operatorname{ccc} 4$

dih 4

cc5

$\operatorname{ccc} 5$

dih5

cc 6

$\operatorname{ccc} 6$

dih 6
$1 \operatorname{ccn} 3$

$2 \operatorname{ccC} 4$

$3 \operatorname{ccc} 5$

$4 \operatorname{ccc} 6$

$5 \operatorname{ccC} 7$

2 cnc 8

$1 \operatorname{ccn} 9$

$8 \operatorname{ccc} 10$

2 hccll

2 hccl2

3 hec13

3 hccl 4

3 hcc15

3 hccl 6

$1 \mathrm{hcn} 17$

$1 \mathrm{hcn} 18$

8 hec19

9 hcc20

9 hcc21

4 hcc22

4 hcc23

5 hcc 24

6 hcc25

6 h⿻c2 6

$1 \operatorname{din} 4$

2 dih5

3 dih 6

4 dih 7

3 dih8

2 dih9

$1 \operatorname{dih} 10$

1 dihl1

1 dih 12

2 dih13

2 dih14

4 dih15

4 dih16

$2 \operatorname{dih} 17$

2 dih18

1 dih19

$8 \operatorname{dih} 20$

8 dih21

3 dih22

$3 \operatorname{din} 23$

4 dih 24

5 dih 25

5 dih26 


$\begin{array}{lc}\text { cc7 } & 1.497530 \\ \text { ccc7 } & 113.370 \\ \text { dih7 } & 177.358 \\ \text { cn8 } & 1.461277 \\ \text { cnc8 } & 114.931 \\ \text { dih8 } & -74.900 \\ \text { cc9 } & 1.508577 \\ \text { ccn9 } & 110.754 \\ \text { dih9 } & -176.377 \\ \text { cc10 } & 1.362540 \\ \text { ccc10 } & 122.692 \\ \text { dih10 } & -144.382 \\ \text { hc11 } & 1.095335 \\ \text { hcc11 } & 109.609 \\ \text { dih11 } & -175.366 \\ \text { hc12 } & 1.096127 \\ \text { hcc12 } & 109.940 \\ \text { dih12 } & 67.837 \\ \text { hc13 } & 1.094495 \\ \text { hcc13 } & 110.652 \\ \text { dih13 } & 175.649 \\ \text { hc14 } & 1.098311 \\ \text { hcc14 } & 108.990 \\ \text { dih14 } & -67.617 \\ \text { hc15 } & 1.094295 \\ \text { hcc15 } & 110.881 \\ \text { dih15 } & -176.129 \\ \text { hc16 } & 1.097823 \\ \text { hcc16 } & 108.822 \\ \text { dih16 } & 66.811 \\ \text { hc17 } & 1.096883 \\ \text { hcn17 } & 107.344 \\ \text { dih17 } & -57.478 \\ \text { hc18 } & 1.103725 \\ \text { hcn18 } & 114.310 \\ \text { dih18 } & 60.483 \\ \text { hc19 } & 1.085947 \\ \text { hcc19 } & 114.903 \\ \text { dih19 } & 58.218 \\ \text { hc20 } & 1.083433 \\ \text { hcc20 } & 121.495 \\ \text { dih20 } & -168.788 \\ \text { hc21 } & 1.085280 \\ \text { hcc21 } & 121.362 \\ \text { dih21 } & 15.624 \\ \text { hc22 } & 1.096526 \\ \text { hcc22 } & 109.952 \\ \text { dih22 } & 68.145 \\ \text { hc23 } & 1.095198 \\ \text { hcc23 } & 110.445 \\ \text { dih23 } & -174.584 \\ \text { hc24 } & 1.102381 \\ \text { hcc24 } & 107.316 \\ \text { dih24 } & -62.003 \\ \text { hc25 } & 1.084127 \\ \text { hcc25 } & 118.107 \\ \text { dih25 } & 156.184 \\ \text { } & \end{array}$




$\begin{array}{lc}\text { hc26 } & 1.083878 \\ \text { hcc26 } & 119.115 \\ \text { dih26 } & 7.029\end{array}$

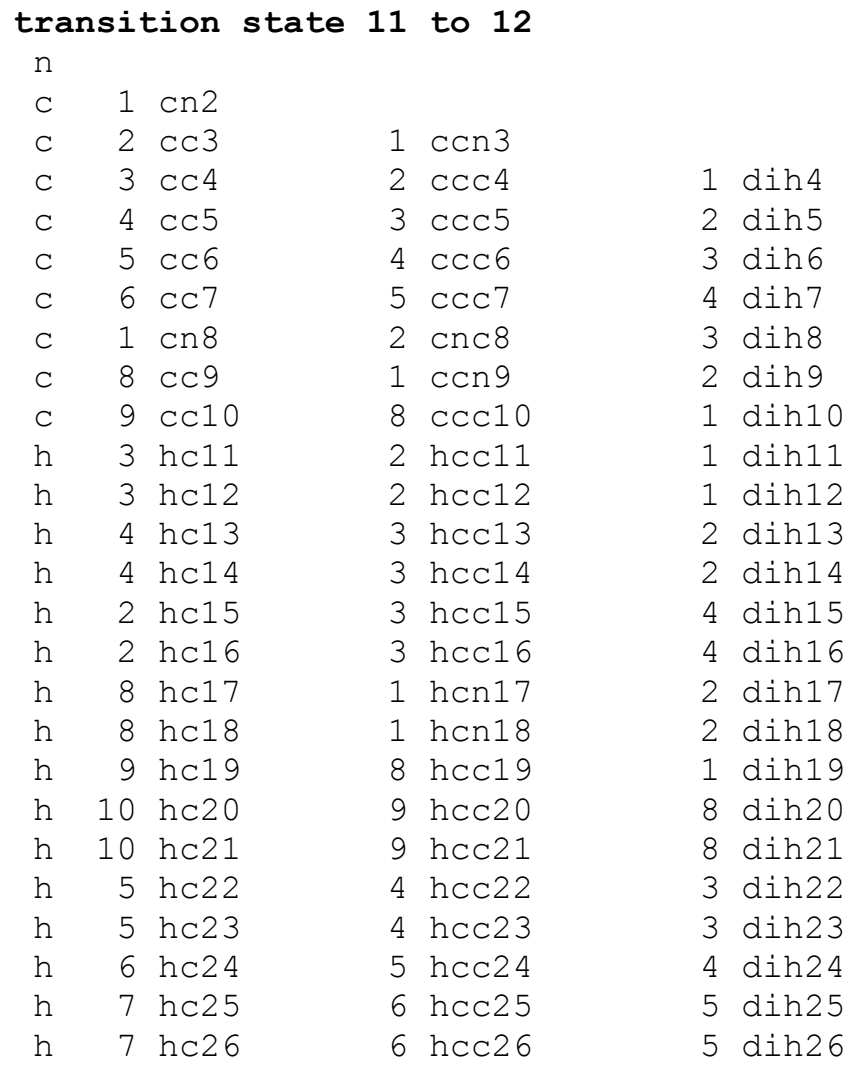

$\begin{array}{lc}\text { cn2 } & 1.464241 \\ \text { cc3 } & 1.539603 \\ \text { ccn3 } & 114.278 \\ \text { cc4 } & 1.536000 \\ \text { ccc4 } & 110.381 \\ \text { dih4 } & -53.287 \\ \text { cc5 } & 1.536154 \\ \text { ccc5 } & 111.106 \\ \text { dih5 } & 52.516 \\ \text { cc6 } & 1.543420 \\ \text { ccc6 } & 110.991 \\ \text { dih6 } & -53.258 \\ \text { cc7 } & 1.497530 \\ \text { ccc7 } & 113.370 \\ \text { dih7 } & 177.358 \\ \text { cn8 } & 1.461277 \\ \text { chc } 8 & 114.931 \\ \text { dih8 } & -74.900 \\ \text { cc9 } 9 & 1.508577 \\ \text { ccn9 } & 110.754 \\ \text { dih9 } & -176.377 \\ \text { cc10 } & 1.362540 \\ \text { ccc10 } & 122.692 \\ \text { dih10 } & -144.382 \\ \text { hc11 } & 1.095335 \\ & \end{array}$




\begin{tabular}{lc} 
hcc11 & 109.609 \\
dih11 & -175.366 \\
hc12 & 1.096127 \\
hcc12 & 109.940 \\
dih12 & 67.837 \\
hc13 & 1.094495 \\
hcc13 & 110.652 \\
dih13 & 175.649 \\
hc14 & 1.098311 \\
hcc14 & 108.990 \\
dih14 & -67.617 \\
hc15 & 1.094295 \\
hcc15 & 110.881 \\
dih15 & -176.129 \\
hc16 & 1.097823 \\
hcc16 & 108.822 \\
dih16 & 66.811 \\
hc17 & 1.096883 \\
hcn17 & 107.344 \\
dih17 & -57.478 \\
hc18 & 1.103725 \\
hcn18 & 114.310 \\
dih18 & 60.483 \\
hc19 & 1.085947 \\
hcc19 & 114.903 \\
dih19 & 58.218 \\
hc20 & 1.083433 \\
hcc20 & 121.495 \\
dih20 & -168.788 \\
hc21 & 1.085280 \\
hcc21 & 121.362 \\
dih21 & 15.624 \\
hc22 & 1.096526 \\
hcc22 & 109.952 \\
dih22 & 68.145 \\
hc23 & 1.095198 \\
hcc23 & 110.445 \\
dih23 & -174.584 \\
hc24 & 1.102381 \\
hcc24 & 107.316 \\
dih24 & -62.003 \\
hc25 & 1.084127 \\
hcc25 & 118.107 \\
dih25 & 156.184 \\
hc26 & 1.083878 \\
hcc26 & 119.115 \\
dih26 & 7.029 \\
& \\
\hline &
\end{tabular}

transition state 13 to 14

C

c $\quad 1 \quad \mathrm{cc} 2$

c $2 \mathrm{cc} 3 \quad 1 \mathrm{ccc} 3$

n 3 nc4 $\quad 2$ ncc4 1 dih4

c 4 cn5 $\quad 3$ cnc5 2 dih5

$\begin{array}{llll}\text { c } & 5 \mathrm{cc} 6 & 4 \operatorname{ccn} 6 & 3 \operatorname{dih} 6\end{array}$

$\begin{array}{llll}\text { c } & 6 \mathrm{CC} 7 & 5 \mathrm{ccC} 7 & 4 \operatorname{dih} 7\end{array}$

$\begin{array}{llll}\text { C } & 7 \operatorname{cc} 8 & 6 \operatorname{ccc} 8 & 5 \operatorname{dih} 8\end{array}$ 


\begin{tabular}{|c|c|c|c|}
\hline$C$ & $7 \operatorname{cc} 9$ & $6 \operatorname{ccc} 9$ & 5 dih 9 \\
\hline C & $9 \mathrm{ccl} 0$ & $7 \operatorname{ccc} 10$ & 6 dih 10 \\
\hline C & $10 \mathrm{cc} 11$ & $9 \operatorname{ccc} 11$ & 7 dih11 \\
\hline C & $7 \mathrm{cc} 12$ & $6 \operatorname{ccc} 12$ & $5 \operatorname{dih} 12$ \\
\hline $\mathrm{h}$ & 6 hc13 & 5 hсc13 & 4 dih13 \\
\hline $\mathrm{h}$ & 6 hcl 4 & 5 hсc14 & 4 dih14 \\
\hline $\mathrm{h}$ & 5 hc15 & $4 \operatorname{hen} 15$ & 2 dih15 \\
\hline $\mathrm{h}$ & 5 hcl 6 & $4 \operatorname{hcn} 16$ & $2 \operatorname{dih} 16$ \\
\hline $\mathrm{h}$ & 3 hcl 7 & $2 \operatorname{hcc} 17$ & $1 \operatorname{dih} 17$ \\
\hline $\mathrm{h}$ & 3 hc1 8 & $2 \mathrm{hcc} 18$ & $1 \operatorname{dih} 18$ \\
\hline $\mathrm{h}$ & 2 hcl 9 & $4 \operatorname{hcn} 19$ & 5 dih19 \\
\hline $\mathrm{h}$ & 1 hc20 & 2 hсc20 & $4 \operatorname{dih} 20$ \\
\hline h & 1 hc21 & 2 hсc21 & 4 dih21 \\
\hline $\mathrm{h}$ & 9 hс22 & 7 hсc22 & 6 dih22 \\
\hline $\mathrm{h}$ & 9 hс23 & 7 hсc23 & 6 dih23 \\
\hline 1 & 10 hc24 & 9 hсc 24 & 7 dih24 \\
\hline $\mathrm{h}$ & 11 hc25 & 10 hcc 25 & 9 dih25 \\
\hline $\mathrm{h}$ & 11 hc26 & 10 hсc 26 & 9 dih26 \\
\hline $\mathrm{h}$ & 12 hc27 & 7 hсc27 & 6 dih27 \\
\hline $\mathrm{h}$ & 12 hc28 & 7 hсc28 & 6 dih28 \\
\hline $\mathrm{h}$ & 12 hc29 & 7 hсc29 & $6 \operatorname{din} 29$ \\
\hline h & 8 hc30 & 7 hсc30 & $6 \operatorname{dih} 30$ \\
\hline $\mathrm{h}$ & 8 hc31 & 7 hсc31 & $6 \operatorname{dih} 31$ \\
\hline $\mathrm{h}$ & 8 hс32 & 7 hсc 32 & $6 \operatorname{din} 32$ \\
\hline $\mathrm{cc} 2$ & & 1.393903 & \\
\hline $\mathrm{cc} 3$ & & 1.489701 & \\
\hline $\mathrm{CCC} 3$ & & 124.924 & \\
\hline nc4 & & 1.438765 & \\
\hline $\mathrm{ncC} 4$ & & 74.621 & \\
\hline dih4 & & 99.272 & \\
\hline cn5 & & 1.459562 & \\
\hline cnc5 5 & & 114.272 & \\
\hline dih5 & & 99.802 & \\
\hline $\operatorname{cc} 6$ & & 1.533462 & \\
\hline $\operatorname{con} 6$ & & 114.463 & \\
\hline dih 6 & & -153.360 & \\
\hline $\mathrm{CC} 7$ & & 1.554670 & \\
\hline $\mathrm{CCC} 7$ & & 119.184 & \\
\hline $\operatorname{dih} 7$ & & -63.063 & \\
\hline $\operatorname{cc} 8$ & & 1.539738 & \\
\hline $\operatorname{ccc} 8$ & & 111.072 & \\
\hline dih8 & & 74.243 & \\
\hline $\mathrm{CC} 9$ & & 1.562214 & \\
\hline $\operatorname{ccc} 9$ & & 105.783 & \\
\hline dih9 & & -166.965 & \\
\hline cc10 & & 1.502803 & \\
\hline $\operatorname{ccc} 10$ & & 116.444 & \\
\hline dih10 & & -177.303 & \\
\hline cc11 & & 1.330447 & \\
\hline $\operatorname{ccc} 11$ & & 125.082 & \\
\hline dih11 & & 118.806 & \\
\hline cc12 & & 1.539854 & \\
\hline $\operatorname{ccc} 12$ & & 110.599 & \\
\hline $\operatorname{dih} 12$ & & -47.867 & \\
\hline hc13 & & 1.097668 & \\
\hline hecl 3 & & 106.103 & \\
\hline
\end{tabular}




$\begin{array}{lc}\text { dih13 } & 175.165 \\ \text { hc14 } & 1.096507 \\ \text { hcc14 } & 108.434 \\ \text { dih14 } & 61.499 \\ \text { hc15 } & 1.103413 \\ \text { hcn15 } & 112.154 \\ \text { dih15 } & 29.234 \\ \text { hc16 } & 1.099954 \\ \text { hcn16 } & 106.368 \\ \text { dih16 } & 144.008 \\ \text { hc17 } & 1.089134 \\ \text { hcc17 } & 115.987 \\ \text { dih17 } & -10.615 \\ \text { hc18 } & 1.093356 \\ \text { hcc18 } & 116.591 \\ \text { dih18 } & -146.747 \\ \text { hc19 } & 1.083590 \\ \text { hcn19 } & 106.312 \\ \text { dih19 } & 5.225 \\ \text { hc20 } & 1.082364 \\ \text { hcc20 } & 120.791 \\ \text { dih20 } & -120.944 \\ \text { hc21 } & 1.083014 \\ \text { hcc21 } & 121.144 \\ \text { dih21 } & 54.328 \\ \text { hc22 } & 1.096101 \\ \text { hcc22 } & 108.382 \\ \text { dih22 } & -54.225 \\ \text { hc23 } & 1.098937 \\ \text { hcc23 } & 107.721 \\ \text { dih23 } & 60.713 \\ \text { hc24 } & 1.088283 \\ \text { hcc24 } & 116.515 \\ \text { dih24 } & -62.451 \\ \text { hc25 } & 1.084509 \\ \text { hcc25 } & 121.729 \\ \text { dih25 } & 178.948 \\ \text { hc26 } & 1.086200 \\ \text { hcc26 } & 121.566 \\ \text { dih26 } & -0.895 \\ \text { hc27 } & 1.092678 \\ \text { hcc27 } & 111.166 \\ \text { dih27 } & -175.184 \\ \text { hc28 } & 1.095734 \\ \text { hcc28 } & 111.508 \\ \text { dih28 } & -54.842 \\ \text { hc29 } & 1.092652 \\ \text { hcc29 } & 111.129 \\ \text { dih29 } & 65.866 \\ \text { hc30 } & 1.093289 \\ \text { hcc30 } & 110.846 \\ \text { dih30 } & 178.983 \\ \text { hc31 } & 1.089604 \\ \text { hcc31 } & 110.710 \\ \text { dih31 } & -59.535 \\ \text { hc32 } & 1.095130 \\ \text { hcc32 } & 110.683 \\ & \\ & \end{array}$


$\operatorname{dih} 32 \quad 59.822$

transition state 14 to 15

$\mathrm{C}$

C $\quad 1 \quad \mathrm{CC} 2$

c $2 \mathrm{cc} 3$

n 3 nc4

c 4 cn 5

C $5 \mathrm{cc} 6$

C $\quad 6 \mathrm{CC} 7$

C $7 \mathrm{CC} 8$

c $8 \mathrm{cc} 9$

C $9 \mathrm{CCl} 0$

C $7 \mathrm{CC} 11$

C $7 \mathrm{CC} 12$

h $6 \mathrm{hcl} 3$

h $6 \mathrm{hcl} 4$

h 5 hel 5

h 5 hel 6

h 3 hel

h 3 hel 8

h 2 hel 9

h 1 hc20

h 1 hc2 1

h 8 hc22

h 8 hc23

h 9 hc24

h 10 hc2 5

h 10 hc26

h 12 hc27

h 12 hc28

h 12 hc29

h 11 hc30

h 11 hc31

h 11 hc32

$1 \mathrm{CCC} 3$

2 ncc 4

3 enc 5

$4 \operatorname{ccn} 6$

$5 \operatorname{ccc} 7$

$6 \operatorname{ccc} 8$

$7 \operatorname{ccc} 9$

$8 \operatorname{ccc} 10$

$6 \mathrm{CCC} 11$

$6 \mathrm{CCC} 12$

5 hccl 3

5 hccl 4

4 hen 15

4 hen16

2 hccl 17

2 hccl 8

3 hccl 9

2 hcc20

2 hcc21

7 hсc22

7 hcc 23

8 hcc 24

9 hcc25

9 hcc26

7 hсc 27

7 hcc 28

7 hcc 29

7 hcc 30

7 hсc31

7 hcc32

1 dih 4

2 dih5

3 dih 6

$4 \operatorname{dih} 7$

5 dih8

6 dih9

7 dih10

5 dih11

5 dih12

4 dih13

4 dih14

3 dih15

3 dih16

1 dih17

1 dih18

4 dih19

3 dih20

3 dih21

6 dih22

6 dih23

7 dih24

8 dih25

8 dih26

6 dih27

6 dih28

6 dih2 9

6 dih30

6 dih31

6 dih32

$\begin{array}{ll}\text { cc2 } & 1.328794 \\ \text { cc3 } & 1.505983 \\ \text { ccc3 } & 124.575 \\ \text { nc4 } & 1.463676 \\ \text { ncc } 4 & 110.746 \\ \text { dih4 } & 131.665 \\ \text { cn5 } & 1.457417 \\ \text { cnc5 } & 112.989 \\ \text { dih5 } & -152.571 \\ \text { cc6 } & 1.538410 \\ \text { ccn6 } & 117.503 \\ \text { dih6 } & -62.408 \\ \operatorname{cc} 7 & 1.543183 \\ \text { ccc7 } & 114.463 \\ \text { dih7 } & -62.989 \\ \operatorname{cc} 8 & 1.548083 \\ \operatorname{ccc} 8 & 110.412 \\ \text { dih8 } & 54.112 \\ \operatorname{cc} 9 & 1.519179 \\ \operatorname{ccc} 9 & 117.785\end{array}$




$\begin{array}{ll}\text { dih9 } & -58.652 \\ \text { cc10 } & 1.381126 \\ \text { ccc10 } & 119.000 \\ \text { dih10 } & 172.217 \\ \text { cc11 } & 1.540076 \\ \text { ccc11 } & 108.681 \\ \text { dih11 } & 172.321 \\ \text { cc12 } & 1.541849 \\ \text { ccc12 } & 110.926 \\ \text { dih12 } & -68.870 \\ \text { hc13 } & 1.096103 \\ \text { hcc13 } & 109.330 \\ \text { dih13 } & 59.551 \\ \text { hc14 } & 1.096546 \\ \text { hcc14 } & 108.514 \\ \text { dih14 } & 174.980 \\ \text { hc15 } & 1.100710 \\ \text { hcn15 } & 106.738 \\ \text { dih15 } & 59.640 \\ \text { hc16 } & 1.095012 \\ \text { hcn16 } & 107.567 \\ \text { dih16 } & 173.071 \\ \text { hc17 } & 1.099335 \\ \text { hcc17 } & 109.228 \\ \text { dih17 } & 10.900 \\ \text { hc18 } & 1.102271 \\ \text { hcc18 } & 109.396 \\ \text { dih18 } & -103.844 \\ \text { hc19 } & 1.086364 \\ \text { hcc19 } & 115.439 \\ \text { dih19 } & -48.289 \\ \text { hc20 } & 1.084297 \\ \text { hcc20 } & 121.722 \\ \text { dih20 } & 179.193 \\ \text { hc21 } & 1.086527 \\ \text { hcc21 } & 121.683 \\ \text { dih21 } & -0.864 \\ \text { hc22 } & 1.097056 \\ \text { hcc22 } & 109.023 \\ \text { dih22 } & 65.700 \\ \text { hc23 } & 1.097776 \\ \text { hcc23 } & 107.682 \\ \text { dih23 } & 179.649 \\ \text { hc24 } & 1.084499 \\ \text { hcc24 } & 116.534 \\ \text { dih24 } & -40.532 \\ \text { hc25 } & 1.083083 \\ \text { hcc25 } & 121.137 \\ \text { dih25 } & 166.165 \\ \text { hc26 } & 1.085166 \\ \text { hcc26 } & 121.382 \\ \text { dih26 } & -19.635 \\ \text { hc27 } & 1.094338 \\ \text { hcc27 } & 110.568 \\ \text { dih27 } & -178.830 \\ \text { hc28 } & 1.094321 \\ \text { hcc28 } & 110.518 \\ & \\ & \end{array}$




$\begin{array}{lc}\text { dih28 } & -59.696 \\ \text { hc29 } & 1.091795 \\ \text { hcc29 } & 112.890 \\ \text { dih29 } & 60.710 \\ \text { hc30 } & 1.094727 \\ \text { hcc30 } & 111.050 \\ \text { dih30 } & -179.878 \\ \text { hc31 } & 1.094460 \\ \text { hcc31 } & 111.231 \\ \text { dih31 } & -59.751 \\ \text { hc32 } & 1.094210 \\ \text { hcc32 } & 110.944 \\ \text { dih32 } & 60.290\end{array}$

transition state 15 to 16

C

C $\quad 1 \quad \mathrm{CC} 2$

n 2 nc3

c $3 \mathrm{cn} 4$

C 4 cc5

c $5 \mathrm{cc} 6$

c $\quad 2 \mathrm{CC} 7$

c $3 \mathrm{cn} 8$

c 8 CC 9

c $9 \mathrm{ccl} 10$

c $5 \mathrm{cc} 11$

C $5 \mathrm{cc} 12$

h 6 hc13

h 6 hc14

h 4 hc15

h 4 hc16

h 8 hc17

h 8 hcl 8

h 9 hc19

h 10 hc20

h 10 hc21

h 1 hc22

h 1 hc23

h 2 hc24

h 7 hc25

h 7 hc26

h 11 hc27

h 11 hc28

h 11 hc2 9

h 12 hc30

h 12 hc31

h 12 hc32

$\begin{array}{ll}\text { cc2 } & 1.541386 \\ \text { nc3 } & 1.478122 \\ \text { ncc3 } & 111.909 \\ \text { cn } 4 & 1.459038 \\ \text { cnc4 } & 113.036 \\ \text { dih4 } & -53.249 \\ \text { cc5 } & 1.558130 \\ \text { ccn5 } & 117.430\end{array}$

$1 \mathrm{ncc} 3$

2 cnc4 1 dih4

3 cen5 2 dih5

$4 \operatorname{ccc} 6 \quad 3$ dih 6

$3 \operatorname{ccn} 7 \quad 4 \operatorname{dih} 7$

$2 \operatorname{cnc} 8 \quad 1$ dih8

$3 \operatorname{con} 9 \quad 2 \operatorname{dih} 9$

$8 \operatorname{ccc} 10 \quad 3 \operatorname{dih} 10$

$4 \operatorname{cccl1} 3$ dih11

$4 \operatorname{ccc} 12 \quad 3 \operatorname{dih} 12$

5 hcc13 4 dih13

5 hcc14 4 dih14

$3 \operatorname{hon} 15 \quad 2$ dih15

$3 \operatorname{hon} 16 \quad 2 \operatorname{dih} 16$

$3 \operatorname{hon} 17 \quad 2 \operatorname{dih} 17$

3 hen18 2 dih18

8 hec19 3 dihl9

9 hcc20 8 dih20

9 hcc21 8 dih21

2 hcc22 3 dih22

2 hcc23 3 dih23

3 hen $24 \quad 4$ dih24

$2 \operatorname{hcc} 25 \quad 3 \operatorname{din} 25$

2 hcc26 3 dih26

5 hcc27 4 dih27

5 hcc28 4 dih28

5 hcc29 4 dih2 9

$5 \operatorname{hcc} 30 \quad 4 \operatorname{dih} 30$

5 hcc31 4 dih31

$5 \operatorname{hcc} 32 \quad 4 \operatorname{dih} 32$ 


$\begin{array}{ll}\text { dih5 } & 51.011 \\ \text { cc6 } & 1.548579 \\ \text { ccc6 } & 108.198 \\ \text { dih6 } & -47.504 \\ \text { cc7 } & 1.500384 \\ \text { ccn7 } & 109.125 \\ \text { dih7 } & -179.983 \\ \text { cn8 } & 1.457189 \\ \text { cnc8 } & 112.471 \\ \text { dih8 } & 83.869 \\ \text { cc9 } & 1.508672 \\ \text { ccn9 } & 110.212 \\ \text { dih9 } & 55.740 \\ \text { cc10 } & 1.362090 \\ \text { ccc10 } & 122.841 \\ \text { dih10 } & -147.936 \\ \text { cc11 } & 1.538094 \\ \text { ccc11 } & 108.165 \\ \text { dih11 } & -166.046 \\ \text { cc12 } & 1.541138 \\ \text { ccc12 } & 112.145 \\ \text { dih12 } & 74.706 \\ \text { hc13 } & 1.096178 \\ \text { hcc13 } & 109.610 \\ \text { dih13 } & 173.029 \\ \text { hc14 } & 1.098174 \\ \text { hcc14 } & 108.431 \\ \text { dih14 } & -71.412 \\ \text { hc15 } & 1.095264 \\ \text { hcn15 } & 108.507 \\ \text { dih15 } & 176.231 \\ \text { hc16 } & 1.098812 \\ \text { hcn16 } & 106.798 \\ \text { dih16 } & -69.261 \\ \text { hc17 } & 1.096825 \\ \text { hcn17 } & 107.380 \\ \text { dih17 } & 174.603 \\ \text { hc18 } & 1.102830 \\ \text { hcn18 } & 115.062 \\ \text { dih18 } & -67.010 \\ \text { hc19 } & 1.086256 \\ \text { hcc19 } & 114.808 \\ \text { dih19 } & 54.121 \\ \text { hc20 } & 1.083358 \\ \text { hcc20 } & 121.514 \\ \text { dih20 } & -168.796 \\ \text { hc21 } & 1.085336 \\ \text { hcc21 } & 121.339 \\ \text { dih21 } & 15.537 \\ \text { hc22 } & 1.095891 \\ \text { hcc22 } & 109.615 \\ \text { dih22 } & -67.157 \\ \text { hc23 } & 1.095320 \\ \text { hcc23 } & 109.476 \\ \text { dih23 } & 176.475 \\ \text { hc24 } & 1.102091 \\ \text { hcn24 } & 105.511 \\ & \\ & \end{array}$




\begin{tabular}{ll} 
dih24 & \multicolumn{1}{c}{63.018} \\
hc25 & 1.083836 \\
hcc25 & 118.506 \\
dih25 & -86.204 \\
hc26 & 1.083877 \\
hcc26 & 118.997 \\
dih26 & 124.472 \\
hc27 & 1.094520 \\
hcc27 & 110.899 \\
dih27 & 177.633 \\
hc28 & 1.095183 \\
hcc28 & 111.193 \\
dih28 & -62.677 \\
hc29 & 1.094311 \\
hcc29 & 111.331 \\
dih29 & 57.587 \\
hc30 & 1.095014 \\
hcc30 & 110.269 \\
dih30 & 172.988 \\
hc31 & 1.090673 \\
hcc31 & 112.838 \\
dih31 & -66.994 \\
hc32 & 1.094678 \\
hcc32 & 110.962 \\
dih32 & 53.911
\end{tabular}

\begin{tabular}{|c|c|c|c|c|c|c|}
\hline \multicolumn{7}{|c|}{$1 \exp$} \\
\hline \multicolumn{7}{|c|}{$C$} \\
\hline C & 1 & cc2 & & & & \\
\hline C & 2 & $\mathrm{cc} 3$ & 1 & $\operatorname{ccc} 3$ & & \\
\hline C & 3 & $\mathrm{CC} 4$ & 2 & $\operatorname{ccc} 4$ & 1 & $\operatorname{dih} 4$ \\
\hline c & 4 & $\mathrm{cc} 5$ & & $\operatorname{ccc} 5$ & 2 & dih5 \\
\hline C & 5 & $\operatorname{cc} 6$ & & $\operatorname{ccc} 6$ & 3 & $\operatorname{din} 6$ \\
\hline C & 2 & $\mathrm{CC} 7$ & 3 & $\mathrm{CCC} 7$ & 4 & $\operatorname{dih} 7$ \\
\hline c & 7 & $\mathrm{cc} 8$ & 2 & $\operatorname{ccc} 8$ & 3 & dih8 \\
\hline c & 8 & $\mathrm{cc} 9$ & 7 & $\operatorname{ccc} 9$ & 2 & dih 9 \\
\hline c & 9 & $\mathrm{cc} 10$ & 8 & $\operatorname{ccc} 10$ & 7 & $\operatorname{dih} 10$ \\
\hline C & 10 & ccl1 & 9 & $\operatorname{cccl} 1$ & 8 & $\operatorname{dih} 11$ \\
\hline $\mathrm{n}$ & 11 & $\mathrm{nc12}$ & 10 & ncc12 & 9 & $\operatorname{dih} 12$ \\
\hline c & 12 & $\operatorname{cn} 13$ & 11 & $\operatorname{cnc} 13$ & 10 & $\operatorname{dih} 13$ \\
\hline C & 7 & $\mathrm{cc} 14$ & 8 & $\operatorname{ccc} 14$ & 9 & $\operatorname{dih} 14$ \\
\hline C & 14 & Cc15 & 7 & $\operatorname{ccc} 15$ & 8 & dih15 \\
\hline c & 15 & $\operatorname{cc} 16$ & 14 & $\operatorname{ccc} 16$ & 7 & $\operatorname{dih} 16$ \\
\hline C & 16 & $\operatorname{cc} 17$ & 15 & $\operatorname{ccc} 17$ & 14 & dih17 \\
\hline C & 17 & $\operatorname{cc} 18$ & 16 & $\operatorname{ccc} 18$ & 15 & $\operatorname{dih} 18$ \\
\hline C & 18 & $\operatorname{cc} 19$ & 17 & $\operatorname{ccc} 19$ & 16 & $\operatorname{dih} 19$ \\
\hline h & 8 & hc20 & 7 & hcc20 & 14 & $\operatorname{dih} 20$ \\
\hline h & 9 & hc21 & 8 & hсc21 & 7 & $\operatorname{dih} 21$ \\
\hline h & 9 & hс22 & 8 & hсc22 & 7 & $\operatorname{dih} 22$ \\
\hline h & 10 & hc23 & 9 & hcc23 & 8 & $\operatorname{dih} 23$ \\
\hline
\end{tabular}




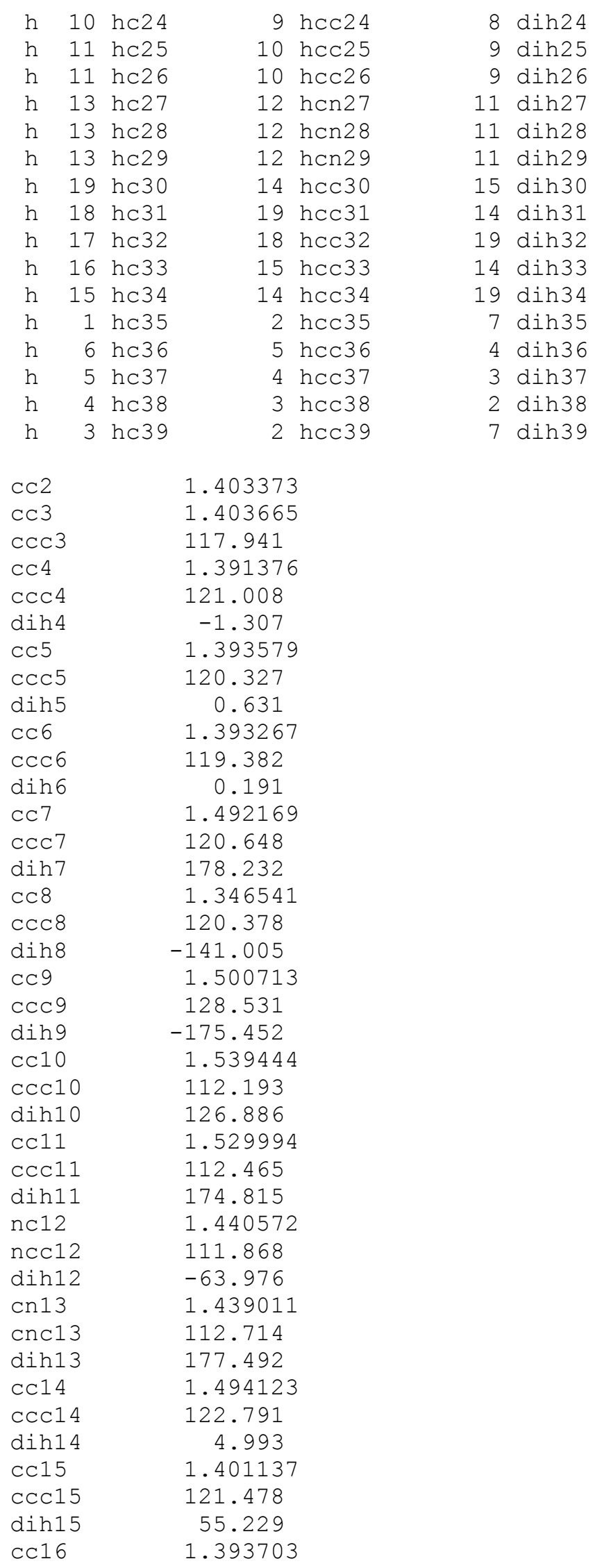




\begin{tabular}{|c|c|}
\hline $\operatorname{ccc} 16$ & 120.920 \\
\hline $\operatorname{dih} 16$ & -179.991 \\
\hline $\operatorname{cc} 17$ & 1.392455 \\
\hline $\operatorname{ccc} 17$ & 120.195 \\
\hline dih17 & 0.023 \\
\hline cc18 & 1.394434 \\
\hline $\operatorname{ccc} 18$ & 119.514 \\
\hline dih1 8 & -0.429 \\
\hline 19 & 1.391139 \\
\hline$=19$ & 120.229 \\
\hline his 19 & 0.010 \\
\hline C20 & 1.088443 \\
\hline hcc20 & 117.008 \\
\hline $\ln 20$ & -176.573 \\
\hline 21 & 1.092996 \\
\hline$=21$ & 110.926 \\
\hline h21 & 4.042 \\
\hline hс22 & 1.097497 \\
\hline hсc22 & 109.809 \\
\hline ih22 & -113.903 \\
\hline$c 23$ & 1.095399 \\
\hline$c c 23$ & 109.780 \\
\hline h23 & -63.260 \\
\hline hc24 & 1.095461 \\
\hline hcc24 & 109.679 \\
\hline $\operatorname{hin} 24$ & 54.100 \\
\hline 25 & 1.105522 \\
\hline$=25$ & 110.198 \\
\hline $\ln 25$ & 58.888 \\
\hline$h c 26$ & 1.104641 \\
\hline 26 & 109.948 \\
\hline $\ln 26$ & 174.401 \\
\hline 27 & 1.102507 \\
\hline $\operatorname{nn} 27$ & 111.231 \\
\hline $\operatorname{dih} 27$ & -60.593 \\
\hline $\mathrm{hc} 28$ & 1.102554 \\
\hline $\mathrm{hcn} 28$ & 111.492 \\
\hline h2 8 & 57.855 \\
\hline 29 & 1.091316 \\
\hline$=n 29$ & 110.435 \\
\hline dih29 & 178.758 \\
\hline hc30 & 1.084267 \\
\hline hcc30 & 119.241 \\
\hline in 30 & 179.083 \\
\hline 31 & 1.084529 \\
\hline cc31 & 119.724 \\
\hline $\operatorname{din} 31$ & -179.333 \\
\hline hс32 & 1.084254 \\
\hline hсc32 & 120.229 \\
\hline h 32 & 179.603 \\
\hline$=33$ & 1.084548 \\
\hline C33 & 119.699 \\
\hline $\operatorname{din} 33$ & -179.647 \\
\hline 34 & 1.084001 \\
\hline & 119.206 \\
\hline 34 & -178.278 \\
\hline & 1.0838 \\
\hline
\end{tabular}




$\begin{array}{ll}\text { hcc35 } & 119.252 \\ \text { dih35 } & 3.014 \\ \text { hc36 } & 1.084606 \\ \text { hcc36 } & 120.074 \\ \text { dih36 } & 178.899 \\ \text { hc37 } & 1.084203 \\ \text { hcc37 } & 120.313 \\ \text { dih37 } & 179.613 \\ \text { hc38 } & 1.084565 \\ \text { hcc38 } & 119.632 \\ \text { dih38 } & -179.312 \\ \text { hc39 } & 1.083756 \\ \text { hcc39 } & 119.333 \\ \text { dih39 } & -1.132\end{array}$

transition state $1 \exp$ to 2exp

C

C $\quad 1 \quad \mathrm{CC} 2$

c $2 \mathrm{Cc} 3$

c $3 \mathrm{cc} 4$

C $4 \mathrm{cc} 5$

c $5 \mathrm{cc} 6$

C $\quad 2 \mathrm{CC} 7$

C $\quad 7 \mathrm{CC} 8$

c $\quad 8$ CC 9

c $\quad 9 \mathrm{cc} 10$

c $\quad 10 \quad \operatorname{cc} 11$

n 11 nc12

c $12 \operatorname{cn} 13$

C $\quad 7 \quad \mathrm{Cc} 14$

C $14 \quad \mathrm{cc} 15$

C $15 \operatorname{cc} 16$

C $16 \quad \mathrm{cc} 17$

c $17 \operatorname{cc} 18$

c $18 \operatorname{cc} 19$

h 8 hc20

h 9 hc21

h 9 hc22

h 10 hc23

h 10 hc24

h 11 hc25

h 11 hc26

h 13 hc27

h 13 hc28

h 13 hc2 9

h 19 hc 30

h 18 hc31

h 17 hc32

h 16 hc33

h 15 hc 34

h 1 hc35

h 6 hc36

h 5 hc37

h 4 hc38

h 3 hc39

$1 \operatorname{ccc} 3$

$2 \operatorname{ccc} 4$

$3 \operatorname{ccc} 5$

$4 \operatorname{ccc} 6$

$3 \operatorname{ccc} 7$

$2 \operatorname{ccc} 8$

$7 \operatorname{ccc} 9$

$8 \operatorname{ccc} 10$

$9 \operatorname{ccc} 11$

10 ncc12

11 cnc13

$8 \operatorname{ccc} 14$

$7 \operatorname{ccc} 15$

$14 \operatorname{ccc} 16$

$15 \operatorname{ccc} 17$

$16 \operatorname{ccc} 18$

$17 \operatorname{ccc} 19$

7 hcc20

8 hcc21

8 hcc22

9 hcc23

9 hcc2 4

10 hcc 25

10 hcc 26

12 hen 27

$12 \operatorname{hen} 28$

12 hen 29

14 hcc 30

19 hcc 31

18 hсc 32

15 hcc 33

14 hcc 34

2 hcc 35

5 hсc 36

4 hcc 37

3 hcc 38

2 hcc 39

1 dih 4

2 dih5

3 dih 6

4 dih 7

3 dih8

2 dih9

7 dih10

8 dih11

9 dih12

10 dih13

9 dih14

8 dih15

7 dih 16

14 dih 17

15 dih18

16 dih19

14 dih20

7 dih21

7 dih22

$8 \operatorname{dih} 23$

8 dih 24

9 dih25

9 dih 26

11 dih27

11 dih28

11 dih29

15 dih 30

14 dih31

19 dih 32

14 dih33

19 dih 34

7 dih 35

4 dih 36

$3 \operatorname{din} 37$

$2 \operatorname{din} 38$

$7 \operatorname{din} 39$

$\mathrm{CC} 2$

1. 408326 


\begin{tabular}{lc} 
cc3 & 1.405133 \\
ccc3 & 117.404 \\
cc4 & 1.392532 \\
ccc4 & 121.298 \\
dih4 & 0.253 \\
cc5 & 1.392354 \\
ccc5 & 120.377 \\
dih5 & 0.234 \\
cc6 & 1.394610 \\
ccc6 & 119.248 \\
dih6 & -0.322 \\
cc7 & 1.482711 \\
ccc7 & 122.905 \\
dih7 & -178.950 \\
cc8 & 1.396221 \\
ccc8 & 123.265 \\
dih8 & 44.339 \\
cc9 & 1.513338 \\
ccc9 & 125.482 \\
dih9 & 19.988 \\
cc10 & 1.537840 \\
ccc10 & 110.170 \\
dih10 & -140.496 \\
cc11 & 1.528661 \\
ccc11 & 106.644 \\
dih11 & 44.720 \\
nc12 & 1.443884 \\
ncc12 & 106.609 \\
dih12 & -51.261 \\
cn13 & 1.443089 \\
cnc13 & 114.701 \\
dih13 & 157.507 \\
cc14 & 1.480214 \\
ccc14 & 119.093 \\
dih14 & -161.100 \\
cc15 & 1.409403 \\
ccc15 & 120.813 \\
dih15 & -144.846 \\
cc16 & 1.390210 \\
ccc16 & 121.332 \\
dih16 & 178.289 \\
cc17 & 1.393921 \\
ccc17 & 120.399 \\
dih17 & 0.553 \\
cc18 & 1.394102 \\
ccc18 & 119.232 \\
dih18 & 0.071 \\
cc19 & 1.389785 \\
ccc19 & 120.416 \\
dih19 & -0.372 \\
hc20 & 1.081406 \\
hcc20 & 115.013 \\
dih20 & -9.989 \\
hc21 & 1.096459 \\
hcc21 & 108.488 \\
dih21 & 98.864 \\
hc22 & 1.090999 \\
& \\
\hline &
\end{tabular}




$\begin{array}{lc}\text { hcc22 } & 111.014 \\ \text { dih22 } & -18.098 \\ \text { hc23 } & 1.094501 \\ \text { hcc23 } & 110.001 \\ \text { dih23 } & -72.996 \\ \text { hc24 } & 1.093403 \\ \text { hcc24 } & 111.643 \\ \text { dih24 } & 167.489 \\ \text { hc25 } & 1.101242 \\ \text { hcc25 } & 110.813 \\ \text { dih25 } & -169.594 \\ \text { hc26 } & 1.105476 \\ \text { hcc26 } & 110.653 \\ \text { dih26 } & 72.581 \\ \text { hc27 } & 1.091807 \\ \text { hcn27 } & 110.063 \\ \text { dih27 } & -165.781 \\ \text { hc28 } & 1.102528 \\ \text { hcn28 } & 114.724 \\ \text { dih28 } & -42.648 \\ \text { hc29 } & 1.100912 \\ \text { hcn29 } & 108.607 \\ \text { dih29 } & 76.983 \\ \text { hc30 } & 1.083343 \\ \text { hcc30 } & 119.558 \\ \text { dih30 } & -177.835 \\ \text { hc31 } & 1.084563 \\ \text { hcc31 } & 119.550 \\ \text { dih31 } & -179.372 \\ \text { hc32 } & 1.084071 \\ \text { hcc32 } & 120.367 \\ \text { dih32 } & 179.963 \\ \text { hc33 } & 1.084586 \\ \text { hcc33 } & 119.561 \\ \text { dih33 } & -179.207 \\ \text { hc34 } & 1.083220 \\ \text { hcc34 } & 119.168 \\ \text { dih34 } & -179.912 \\ \text { hc35 } & 1.083629 \\ \text { hcc35 } & 119.097 \\ \text { dih35 } & -0.678 \\ \text { hc36 } & 1.084557 \\ \text { hcc36 } & 120.052 \\ \text { dih36 } & 179.783 \\ \text { hc37 } & 1.084105 \\ \text { hcc37 } & 120.380 \\ \text { dih37 } & 179.871 \\ \text { hc38 } & 1.084643 \\ \text { hcc38 } & 119.540 \\ \text { dih38 } & -179.402 \\ \text { hc39 } & 1.081548 \\ \text { hcc39 } & 119.545 \\ \text { dih39 } & 2.242 \\ & \end{array}$




\begin{tabular}{|c|c|c|c|}
\hline \multicolumn{4}{|l|}{$3 e x p$} \\
\hline \multicolumn{4}{|l|}{$\begin{array}{c}\text { sexp } \\
C\end{array}$} \\
\hline C & $1 \mathrm{cc} 2$ & & \\
\hline C & $2 \mathrm{cc} 3$ & $1 \operatorname{ccc} 3$ & \\
\hline C & $3 \mathrm{cc} 4$ & $2 \operatorname{ccc} 4$ & $1 \operatorname{dih} 4$ \\
\hline C & $4 \mathrm{cc} 5$ & $3 \operatorname{ccc} 5$ & 2 dih5 \\
\hline C & $5 \operatorname{cc} 6$ & $4 \operatorname{ccc} 6$ & 3 dih 6 \\
\hline C & $5 \mathrm{CC} 7$ & $4 \operatorname{ccc} 7$ & $3 \operatorname{dih} 7$ \\
\hline C & $7 \mathrm{cc} 8$ & $5 \operatorname{ccc} 8$ & 4 dih 8 \\
\hline C & $8 \operatorname{cc} 9$ & $7 \operatorname{ccc} 9$ & 5 dih 9 \\
\hline $\mathrm{C}$ & $9 \mathrm{ccl} 0$ & $8 \operatorname{ccc} 10$ & 7 dih10 \\
\hline C & $10 \mathrm{ccl} 1$ & $9 \operatorname{ccc} 11$ & 8 dih11 \\
\hline $\mathrm{n}$ & $11 \mathrm{nc12}$ & 10 ncc 12 & 9 dih12 \\
\hline C & $12 \mathrm{cn} 13$ & 11 encl3 & 10 dih13 \\
\hline $\mathrm{h}$ & 7 hol 4 & 5 hсc14 & 4 dih14 \\
\hline $\mathrm{h}$ & 8 hcl 5 & 7 hcc15 & 5 dih15 \\
\hline $\mathrm{h}$ & 9 hcl 6 & $8 \mathrm{hcc} 16$ & 7 dih16 \\
\hline $\mathrm{h}$ & 9 hcl 7 & $8 \mathrm{hcc} 17$ & 7 dih 17 \\
\hline $\mathrm{h}$ & 10 hc18 & 9 hсc18 & 8 dih18 \\
\hline $\mathrm{h}$ & 10 hc19 & 9 hсc19 & 8 dih19 \\
\hline $\mathrm{h}$ & 11 hc20 & 10 hcc 20 & 9 dih20 \\
\hline $\mathrm{h}$ & 11 hc21 & 10 hcc 21 & 9 dih21 \\
\hline $\mathrm{h}$ & 13 hс22 & $12 \operatorname{hcn} 22$ & 11 dih22 \\
\hline $\mathrm{h}$ & 13 hс23 & $12 \operatorname{hcn} 23$ & 11 dih23 \\
\hline $\mathrm{h}$ & 13 hс24 & $12 \operatorname{hcn} 24$ & 11 dih24 \\
\hline $\mathrm{h}$ & 6 hc2 5 & 5 hсc 25 & $4 \operatorname{din} 25$ \\
\hline $\mathrm{h}$ & 1 hс26 & $2 \operatorname{hcc} 26$ & 3 dih26 \\
\hline $\mathrm{h}$ & 2 hс27 & $3 \operatorname{hcc} 27$ & 4 dih27 \\
\hline $\mathrm{h}$ & 3 hс2 8 & $2 \operatorname{hcc} 28$ & $1 \operatorname{dih} 28$ \\
\hline $\mathrm{h}$ & 4 hс29 & 3 hсc 29 & $2 \operatorname{dih} 29$ \\
\hline $\mathrm{Cc} 2$ & & 1.394100 & \\
\hline $\mathrm{cc} 3$ & & 1.393010 & \\
\hline $\mathrm{Ccc} 3$ & & 119.415 & \\
\hline $\mathrm{CC} 4$ & & 1.392401 & \\
\hline $\operatorname{ccc} 4$ & & 120.402 & \\
\hline dih4 & & -0.759 & \\
\hline $\mathrm{CC} 5$ & & 1.404161 & \\
\hline $\operatorname{ccc} 5$ & & 120.937 & \\
\hline $\operatorname{dih} 5$ & & -0.335 & \\
\hline $\operatorname{cc} 6$ & & 1.404873 & \\
\hline $\operatorname{ccc} 6$ & & 117.829 & \\
\hline $\operatorname{dih} 6$ & & 1.643 & \\
\hline $\mathrm{CC} 7$ & & 1.475188 & \\
\hline $\mathrm{CCC} 7$ & & 123.151 & \\
\hline $\operatorname{dih} 7$ & & 179.712 & \\
\hline $\operatorname{cc} 8$ & & 1.340567 & \\
\hline $\operatorname{ccc} 8$ & & 128.932 & \\
\hline dih8 & & 37.457 & \\
\hline $\operatorname{cc} 9$ & & 1.501968 & \\
\hline $\operatorname{ccc} 9$ & & 128.997 & \\
\hline dihg & & 2.927 & \\
\hline $\operatorname{cc} 10$ & & 1.537674 & \\
\hline
\end{tabular}




\begin{tabular}{|c|c|}
\hline $\operatorname{ccc} 10$ & 112.257 \\
\hline dih10 & 134.931 \\
\hline ccl1 & 1.529513 \\
\hline $\operatorname{ccc} 11$ & 112.620 \\
\hline dih11 & 175.918 \\
\hline nc12 & 1.440701 \\
\hline $\mathrm{ncc} 12$ & 111.905 \\
\hline $\operatorname{dih} 12$ & -63.854 \\
\hline $\operatorname{cn} 13$ & 1.439075 \\
\hline 1013 & 112.692 \\
\hline dih13 & 178.080 \\
\hline hc14 & 1.088084 \\
\hline hcc14 & 114.260 \\
\hline dih14 & -144.423 \\
\hline 15 & 1.089060 \\
\hline c15 & 116.439 \\
\hline h15 & -179.605 \\
\hline hc16 & 1.093891 \\
\hline hcc16 & 110.641 \\
\hline $\ln 16$ & 12.359 \\
\hline 17 & 1.097855 \\
\hline $\operatorname{cc17}$ & 110.074 \\
\hline h17 & -105.349 \\
\hline hc18 & 1.095493 \\
\hline hсc18 & 109.778 \\
\hline dih1 8 & -62.124 \\
\hline 19 & 1.095516 \\
\hline c19 & 109.607 \\
\hline h19 & 55.131 \\
\hline $\mathrm{hc} 20$ & 1.105625 \\
\hline hcc20 & 110.195 \\
\hline $\ln 20$ & 58.923 \\
\hline 21 & 1.104524 \\
\hline$c 21$ & 109.984 \\
\hline $\operatorname{dih} 21$ & 174.410 \\
\hline hc22 & 1.102573 \\
\hline hen22 & 111.048 \\
\hline in 22 & -62.432 \\
\hline 23 & 1.102410 \\
\hline$n 23$ & 111.665 \\
\hline dih23 & 56.039 \\
\hline hc24 & 1.091337 \\
\hline$=n 24$ & 110.436 \\
\hline $\operatorname{din} 24$ & 177.193 \\
\hline 25 & 1.085259 \\
\hline 25 & 119.048 \\
\hline dih2 5 & 178.747 \\
\hline hс26 & 1.084522 \\
\hline hcc26 & 120.074 \\
\hline h 26 & -179.228 \\
\hline 27 & 1.084158 \\
\hline 27 & 120.293 \\
\hline h 27 & -179.936 \\
\hline 28 & 1.084591 \\
\hline & 120.005 \\
\hline 28 & 178.506 \\
\hline & 1.082686 \\
\hline
\end{tabular}




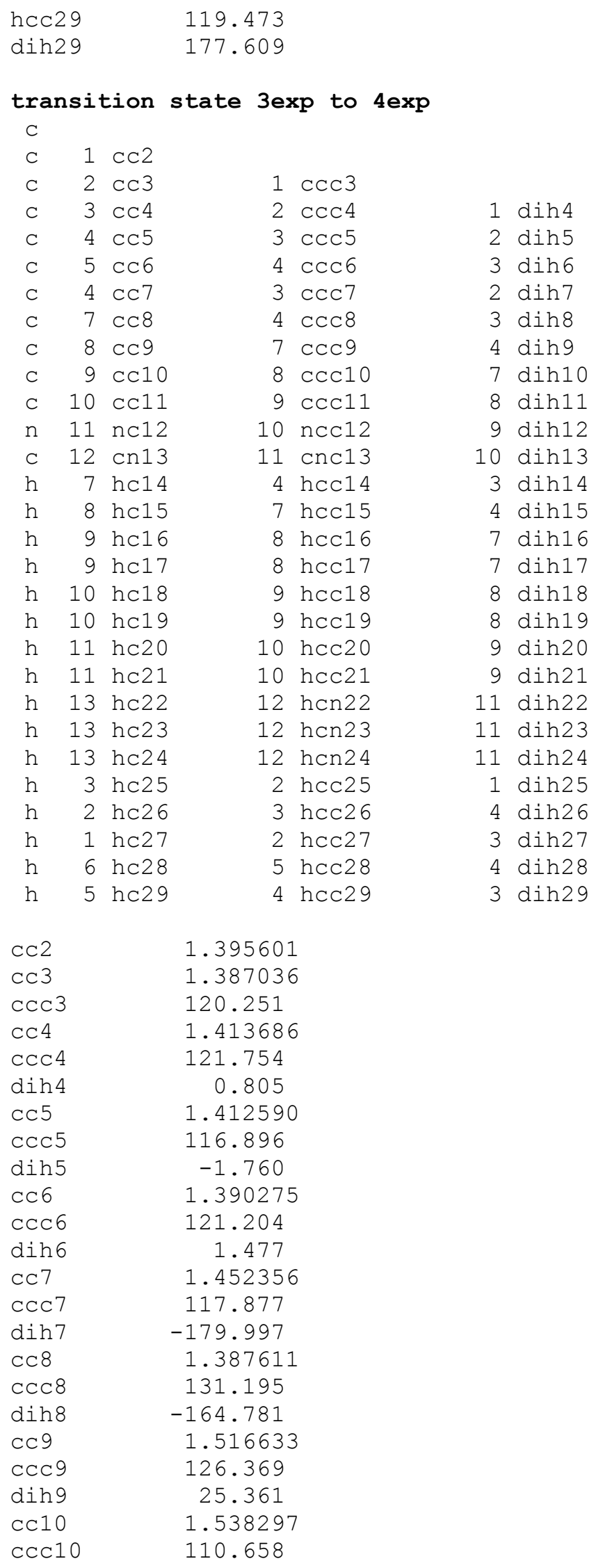




$\begin{array}{lc}\text { dih10 } & -135.820 \\ \text { cc11 } & 1.527636 \\ \text { ccc11 } & 106.485 \\ \text { dih11 } & 43.688 \\ \text { nc12 } & 1.44499 \\ \text { ncc12 } & 106.500 \\ \text { dih12 } & -51.692 \\ \text { cn13 } & 1.443910 \\ \text { cnc13 } & 114.067 \\ \text { dih13 } & 156.274 \\ \text { hc14 } & 1.087420 \\ \text { hcc14 } & 113.493 \\ \text { dih14 } & 17.901 \\ \text { hc15 } & 1.083857 \\ \text { hcc15 } & 114.051 \\ \text { dih15 } & 175.703 \\ \text { hc16 } & 1.095673 \\ \text { hcc16 } & 108.050 \\ \text { dih16 } & 103.536 \\ \text { hc17 } & 1.090310 \\ \text { hcc17 } & 111.061 \\ \text { dih17 } & -12.791 \\ \text { hc18 } & 1.094335 \\ \text { hcc18 } & 109.921 \\ \text { dih18 } & -73.867 \\ \text { hc19 } & 1.093452 \\ \text { hcc19 } & 111.720 \\ \text { dih19 } & 166.581 \\ \text { hc20 } & 1.101121 \\ \text { hcc20 } & 110.954 \\ \text { dih20 } & -170.083 \\ \text { hc21 } & 1.105212 \\ \text { hcc21 } & 110.761 \\ \text { dih21 } & 71.809 \\ \text { hc22 } & 1.092416 \\ \text { hcn22 } & 110.126 \\ \text { dih22 } & -169.350 \\ \text { hc23 } & 1.101857 \\ \text { hcn23 } & 114.302 \\ \text { dih23 } & -46.830 \\ \text { hc24 } & 1.100440 \\ \text { hcn24 } & 108.742 \\ \text { dih24 } & 72.906 \\ \text { hc25 } & 1.085147 \\ \text { hcc25 } & 119.573 \\ \text { dih25 } & -179.807 \\ \text { hc26 } & 1.084553 \\ \text { hcc26 } & 119.695 \\ \text { dih26 } & -179.436 \\ \text { hc27 } & 1.083894 \\ \text { hcc27 } & 120.437 \\ \text { dih27 } & 179.796 \\ \text { hc28 } & 1.084696 \\ \text { hcc28 } & 119.379 \\ \text { dih28 } & -179.656 \\ \text { hc29 } & 1.080942 \\ \text { hcc29 } & 120.055 \\ & \\ & \end{array}$




\section{Energetic Results}

\begin{tabular}{|c|c|c|}
\hline & b3lyp/6-311g** & \\
\hline 1 & -446.6502 & -448.0778 \\
\hline ts 1 to 2 & -446.6437 & -448.0685 \\
\hline 2 & -446.6734 & -448.0926 \\
\hline ts 2 to $3 a$ & -446.6552 & -448.0804 \\
\hline ts 2 to $3 b$ & -446.6501 & -448.0748 \\
\hline $3 a$ & -446.6765 & -448.1068 \\
\hline $3 b$ & -446.6794 & -448.1099 \\
\hline ts $3 a$ to $4 a$ & -446.6634 & -448.0981 \\
\hline ts $3 a$ to $4 b$ & -446.6604 & -448.0948 \\
\hline ts $3 b$ to $4 c$ & -446.6661 & -448.1043 \\
\hline ts $3 b$ to $4 d$ & -446.6661 & -448.1003 \\
\hline $4 a$ & -446.6997 & -448.1417 \\
\hline $4 b$ & -446.6979 & -448.1391 \\
\hline $4 c$ & -446.6982 & -448.1394 \\
\hline 4d & -446.6981 & -448.1391 \\
\hline 5 & -485.9727 & -487.5364 \\
\hline ts 5 to 6 & -485.9662 & -487.5271 \\
\hline 6 & -485.9960 & -487.5512 \\
\hline ts 6 to 7 & -485.9622 & -487.5242 \\
\hline 7 & -485.9994 & -487.5681 \\
\hline ts 7 to 8 & -485.9915 & -487.5637 \\
\hline 8 & -486.0266 & -487.6053 \\
\hline 9 & -407.3279 & -408.6173 \\
\hline ts 9 to 10 & -407.3209 & -408.6075 \\
\hline 10 & -407.3495 & -408.6309 \\
\hline ts 10 to 11 & -407.3214 & -408.6080 \\
\hline 11 & -407.3547 & -408.6498 \\
\hline ts 11 to 12 & -407.3459 & -408.6450 \\
\hline 12 & -407.3799 & -408.6853 \\
\hline 13 & -485.9706 & -487.5343 \\
\hline ts 13 to 14 & -485.9636 & -487.5245 \\
\hline 14 & -485.9922 & -487.5478 \\
\hline ts 14 to 15 & -485.9649 & -487.5270 \\
\hline 15 & -486.0022 & -487.5709 \\
\hline ts 15 to 16 & -485.9915 & -487.5637 \\
\hline 16 & -486.0274 & -487.6066 \\
\hline
\end{tabular}

